UCRL-ID-142553

\title{
Advanced Wavefront Control Techniques
}

S. S. Olivier, J. M. Brase, K. Avicola, C. A. Thompson, M. W. Kartz, S. Winters, R. Hartley, J. Wilhelmsen, F. U. Dowla, C. J. Carrano, B. J. Bauman, D. M. Pennington, D. Lande, R. M. Sawvel, D. A. Silva, J. B. Cooke, C. G. Brown

\section{February 21, 2001}

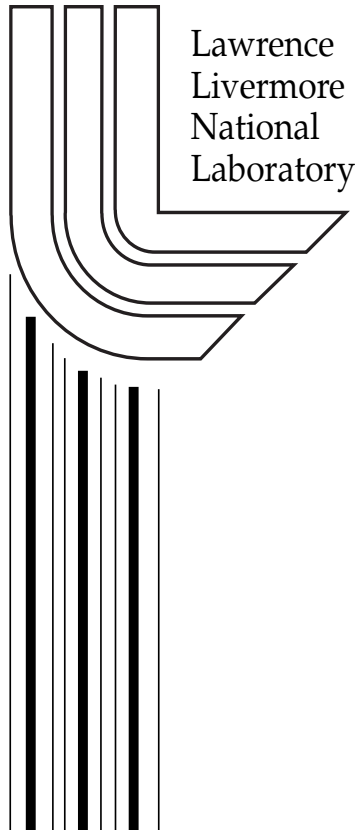




\section{DISCLAIMER}

This document was prepared as an account of work sponsored by an agency of the United States Government. Neither the United States Government nor the University of California nor any of their employees, makes any warranty, express or implied, or assumes any legal liability or responsibility for the accuracy, completeness, or usefulness of any information, apparatus, product, or process disclosed, or represents that its use would not infringe privately owned rights. Reference herein to any specific commercial product, process, or service by trade name, trademark, manufacturer, or otherwise, does not necessarily constitute or imply its endorsement, recommendation, or favoring by the United States Government or the University of California. The views and opinions of authors expressed herein do not necessarily state or reflect those of the United States Government or the University of California, and shall not be used for advertising or product endorsement purposes.

This work was performed under the auspices of the U.S. Department of Energy by the University of California, Lawrence Livermore National Laboratory under Contract No. W-7405-Eng-48.

This report has been reproduced

directly from the best available copy.

Available to DOE and DOE contractors from the

Office of Scientific and Technical Information

P.O. Box 62, Oak Ridge, TN 37831

Prices available from (423) 576-8401

http://apollo.osti.gov/bridge/

Available to the public from the

National Technical Information Service

U.S. Department of Commerce 5285 Port Royal Rd., Springfield, VA 22161 http://www.ntis.gov/

OR

Lawrence Livermore National Laboratory Technical Information Department's Digital Library

http://www.llnl.gov/tid/Library.html 


\section{Advanced Wavefront Control Techniques \\ 98-ERD-061 \\ Final Report}

S. S. Olivier, J. M Brase, K. Avicola, C.A. Thompson, M.W. Kartz, S. Winters, R. Hartley, J. Wilhelmsen, F. U. Dowla, C.J. Carrano, B. J. Bauman, D. M. Pennington, D. Lande, R.M. Sawvel, D.A. Silva, J. B. Cooke, C. G. Brown,

\section{Summary}

Programs at LLNL that involve large laser systems - ranging from the National Ignition Facility to new tactical laser weapons - depend on the maintenance of laser beam quality through precise control of the optical wavefront. This can be accomplished using adaptive optics, which compensate for time-varying aberrations that are often caused by heating in a high-power laser system. Over the past two decades, LLNL has developed a broad capability in adaptive optics technology for both laser beam control and highresolution imaging. This adaptive optics capability has been based on thin deformable glass mirrors with individual ceramic actuators bonded to the back. In the case of high-power lasers, these adaptive optics systems have successfully improved beam quality. However, as we continue to extend our applications requirements, the existing technology base for wavefront control cannot satisfy them. To address this -issue, this project studied improved modeling tools to increase our detailed understanding of the performance of these systems, and evaluated novel approaches to low-order wavefront control that offer the possibility of reduced cost and complexity. We also investigated improved beam control technology for high-resolution wavefront control. Many high-power laser systems suffer from high-spatial-frequency aberrations that require control of hundreds or thousands of phase points to provide adequate correction. However, the cost and size of current deformable mirrors can become prohibitive for applications requiring more than a few tens of phase control points. New phase control technologies are becoming available which offer control of many phase points with small low-cost devices

The largest conventional deformable mirrors currently available have approximately one thousand actuators and cost approximately $\$ 1 \mathrm{M}$. This cost scaling of $\$ 1000$ per actuator causes deformable mirrors to be prohibitively expensive for many applications that require high-spatial-frequency wavefront control. A new approach with the potential to solve this problem is the use of liquid crystal (LC) spatial light modulator (SLM) technology. Current LC SLM technology provides high-spatial-frequency wavefront control, with hundreds of thousands of degrees of freedom, more than two orders of magnitude greater than the largest deformable mirror. Furthermore, even with the increased spatial resolution, the cost of these devices is nearly two orders of magnitude less than the cost of the largest deformable mirror. Thus, LC SLM devices have the potential to create a paradigm shift of four orders of magnitude in the cost per phase control point for advanced adaptive optics.

The goal of this project was to expand our wavefront control capabilities with improved modeling tools, new devices that reduce system cost and complexity, and extensions to high spatial and temporal frequencies using new adaptive optics technologies. In FY 99, the second year of this project, work was performed in four areas (1) advanced modeling tools for deformable mirrors (2) low-order wavefront correctors with Alvarez lenses, (3) a direct phase measuring heterodyne wavefront sensor, and (4) highspatial-frequency wavefront control using spatial light modulators.

The advanced modeling tools for deformable mirrors utilize the finite element method to predict the response of the mirror surface from input forces or displacements on the back of the mirror. The output of the model is the linear displacement of the surface at defined points on the mirror. These points uniquely determine the response of the mirror surface for the displacement of each actuator and may then serve as input to a model of the adaptive optics system. Previous LLNL models used a set of Gaussian approximations or other ad hoc models of the surface response for the displacement of each actuator. The finite element method provides a significant improvement in residual error prediction as compared to Gaussian models. This was experimentally validated on a prototype NIF deformable mirror. A detailed report on the work on improved deformable mirrors is presented in the first chapter of this final report. 
The work on the Alvarez lenses sought to develop a wavefront corrector that (1) had only two or three degrees of freedom, (2) could potentially have lower cost than that for conventional adaptive optics systems, (3) could have greater reliability, and (4) imparted less intensity variations in intermediate field. A variable focal-length lens can be constructed by laterally translating two complementary lenses each having a plano side and a second curved side. When the second surface is a cubic function, the lateral shearing of the two optics produces a variable focal-length lens in the axis of the shear. Focus and astigmatism can be corrected with no change in beam size. We fabricated Alvarez lenses using two different methods. We tested the lenses using interferometry, beam propagation, and far-field imagery. Results confirmed that the design and manufacturing specifications were met. Experimental data also confirmed theoretical performance expectations, indicating the usefulness of these optics, and identifying the resulting differences between the two fabrication methods. A detailed report on the work on Alvarez lenses is presented in the second chapter of this final report.

Current wavefront control systems use a Hartmann sensor, which measures local wavefront slopes, as the wavefront sensor. A heterodyne sensor would directly measure phase, and potentially reduce the cost and complexity of the adaptive optics system compared to the conventional approach. A second feature of this wavefront sensor technique is that it is much higher speed than the Hartmann sensor. In FY 99, we designed, fabricated and tested a 2-channel pulsed demonstration system, and verified that heterodyne signals were obtained when using a phase-modulated pulsed laser. We also designed a sensor array appropriate for control of a 39-actuator deformable mirror. A detailed report on the work on heterodyne wavefront sensing is presented in the third chapter of this final report.

The primary enabling technology for high-spatial-frequency wavefront control is a new generation of spatial light modulators (SLM's), both liquid crystal (LC) and micro-electro-mechanical systems (MEMS). These devices can provide wavefront correction at high spatial frequencies with relatively low unit costs. Some of these devices can also be operated at high temporal frequencies. In FY99, we received LC SLM devices from two vendors: Jenoptik and Hamamatsu. We designed, implemented and tested driver software and the optical addressing source assemblies for both LC SLM devices. We characterized optical wavefront control capabilities of both devices using interferometry, and we assembled and aligned the laboratory test-bed system. We designed, implemented and tested the high-spatial-frequency wavefront sensor and control algorithm, and we performed initial high-spatial-frequency wavefront control experiments in lab test-bed with Jenoptik LC SLM. A detailed report on the work on LC SLM's in FY99 is presented in the fourth chapter of this final report.

During the third and final year of this project, we integrated a LC SLM device from Hamamatsu into the adaptive optics test-bed that was previously developed for this project. This test-bed employs a highresolution wavefront sensor that measures the slope of the wavefront at 2000 points across the aperture. This slope information must then be processed to yield the wavefront phase. In order to reduce the computation time for this phase reconstruction, new algorithms were developed during this project. These algorithms are based on Fourier transform techniques that require fewer computations for systems with large numbers of phase reconstruction points, compared with the traditional matrix-vector multiplication approach. We also studied wavelet techniques to further improve the efficiency of the wavefront reconstruction algorithms. A detailed report on the work on phase reconstruction algorithms is presented in the fifth chapter of this final report.

After integrating the Hamamatsu LC SLM into the test-bed, we performed a detailed characterization of the high-resolution wavefront control performance of this device. These tests successfully demonstrated near diffraction-limited performance. To our knowledge, these are the first quantitative measurements demonstrating near diffraction-limited performance of a wavefront control system using a LC SLM. A detailed report on the work on LC SLM's in FY00 is presented in the sixth chapter of this final report. The combination of the large number of phase-control points and the demonstrated wavefront quality makes this technology a viable choice for high-performance beam control and image compensation applications that require high-order correction beyond the capability of conventional deformable mirrors. Based on the results of this work, new projects have begun to apply this technology to systems for precision laser drilling 
and studies of enhanced human vision. Many other laser and imaging systems could ultimately benefit from this technology.
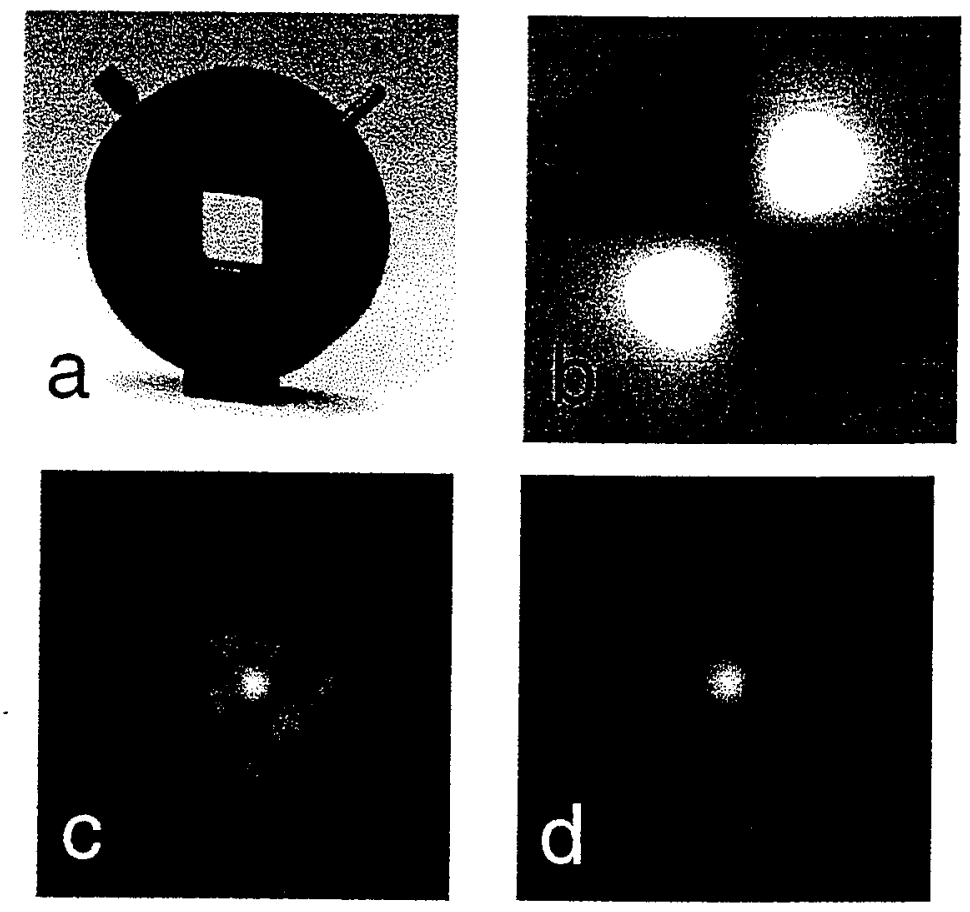

Pictures of (a) Hamamatsu LC SLM, (b) wavefront aberration applied during performance testing, (c) resulting aberrated far-field image, and (d) corrected far-field image. 


\section{Chapter 1 - Improved Deformable Mirrors}

\section{Scott Winters}

\subsection{Introduction}

The scope of work for improved deformable mirrors focused on development of improved modeling tools which could be used in conjunction with existing beam propagation codes. In general, the improved model utilizes the finite element method to predict the response of the mirror surface from input forces or displacements on the back of the mirror. The output of the model is the linear displacement of the surface at defined points on the mirror surface. These points uniquely determine the response of the mirror surface for the displacement of each actuator and may then serve as input to a model of the adaptive optics system. Previous LLNL models use a set of Gaussian approximations or unvalidated models of the surface response for the displacement of each actuator. The finite element method provides a significant improvement in residual error prediction as compared to Gaussian models.

Initial work focused on developing the methodology for an integrated system level adaptive optics simulation tool. Based on experience with various LLNL adaptive optics systems, it was clear that a validated model was lacking. Throughout LLNL, many different beam propagation codes are utilized, so it was important to have flexibility in the modeling tools output file format. This methodology is outlined in Figure 1. Figure 1 depicts various key steps in developing the tool. The methodology starts with defining the physical structure (i.e., deformable mirror), developing the mathematical model, developing the numerical model, assessing the mesh errors, assessing the mathematical model errors with experimental data, post processing the model outputs and lastly supplying the data to various integrated adaptive optics codes.

\subsection{Model Development}

The main focus of this work was to develop a validated computer model which will represent the static surface response of a deformable mirror. Many authors have considered both numerical models, such as finite elements, and analytical models, such as Gaussian functions. The modeling errors associated with using most analytical functions are related to the boundary conditions along the edge and the so called pinning error effect. The pinning error effect occurs when all the actuators are pushed identically, and the model shows a surface that is made of bumps of equal height. This rippled surface is due to modeling errors and does not occur in the real device. Some numerical approaches, such as the finite element method, do not have these deficiencies since they maintain continuity in displacement, slope, and higher derivatives between elements. After a complete evaluation of these different approaches, the finite element method was selected due to its accuracy, flexibility, and the availability of commercial software. Many different finite element programs were evaluated for use, such as, SDRC, MSC, COSMOS-M, Pro-Mechanica and LLNL developed codes, for example. The Structural Dynamics Research Corporation (SDRC) I-DEAS Master Series 5 
modeling package was selected due to its flexibility and ability to define specific output locations. This code was utilized to generate the finite element model of the National Ignition Facility (NIF) deformable mirror.

A solid finite element model was developed of the NIF 39 actuator prototype deformable mirror, which included one facesheet and thirty-nine 3-DOF springs. The model was built using parabolic tetrahedron solid elements, each having 10 nodes with the overall model having 68,323 . To verify that the meshing errors were sufficiently small, models with mesh sizes ranging from 26,000 nodes to 350,000 nodes were developed and solved using the same boundary conditions. This approach follows standard finite element mesh refinement techniques and will produce an assessment of the meshing error. The boundary conditions were free edges, constrained springs, and a force applied at actuator number 20 . The selection of this boundary condition set is justified due to the symmetry in the system. Using surface displacement as the performance metric, approximately ninety-nine percent asymptotic convergence was achieved, as compared to the 350,000 node model, after reaching approximately 68,000 nodes. This convergence indicates a mesh density that is sufficient to capture the displacement spatial frequencies in the model. Therefore, it is concluded that a ninetynine percent convergence is sufficient, based on uncertainties associated with other aspects of the modeling, e.g., material properties and boundary conditions, memory capacity limitations, and computational time considerations. Figure 2 shows an example surface response (influence function) for the center actuator.

With the model complete and the influence functions generated, the next step was to gather experimental results. These experimental results are needed to verify the accuracy of mathematical model so that it may be used with quantifiable. The magnitude of acceptable model accuracy is established by the intended use. With the ultimate goal of using this model for static surface shape control, model errors should be small compared to errors generated by the control law, the magnitude of the aberration to be corrected, etc. Furthermore, model errors should be comparable to uncertainties in material parameters, e.g., elastic modulus, Poisson's ratio. Lastly, once the static model is verified, small variations could be made to the model to complete sensitivity studies for design purposes.

\subsection{Experimental Test}

Experimental tests were completed on the NIF 39 actuator deformable mirror in order to validate the model developed in the previous section. The tests utilized a 610 milli-meter (24 inch) aperture Fizeau interferometer system at Lawrence Livermore National Laboratory (LLNL), which was specially developed to characterize the performance of the NIF adaptive optics system. As with any experiment, understanding the uncertainties and errors are very important. The large interferometer system was characterized through a series of tests, which included accuracy, repeatability, and reproducibility. These parameters are defined based on the International Organization for Standardization (ISO) as follows. Accuracy is defined as the closeness of the agreement between the result of a measurement and a true value of the measurand. Repeatability is defined as the closeness of the agreement between the results of 
successive measurements of the same measurand carried out under the same conditions of measurement. Reproducibility is defined as the closeness of the agreement between the results of measurements of the same measurand carried out under changed conditions of measurement. For these specific tests, repeatability measurements were taken over a few minutes and reproducibility measurements were taken over approximately one hour. The most important characterization for verifying the mathematical model is repeatability, since two measurements are taken and then subtracted to get the experimental influence function. This is accomplished by taking a measurement of the deformable mirror at a nominal voltage condition and then repeating the measurement after increasing the voltage on a single actuator. The difference between these two measurements gives the actuator influence function. Table 1 summarizes the overall characterization test results.

\subsection{Model Verification}

In this section, a comparison between results generated from the finite element model and experiment will be made. This comparison will allow a statement of model fidelity to be made and also gives confidence in using the model for future control implementation.

The comparison between the model and experimental data were made using a normalization approach. Comparisons were made between selected influence functions which give representative locations over the surface. Results for selected actuators can be seen in Table 2, which shows actuator number and percent difference between model and experiment relative to the maximum surface displacement. It should be noted that better translation alignment between experimental and model data would most likely reduce the percent difference. Figure 3 depicts the difference between model and experimental data.

On the basis of the results listed in Table 2, a statement of model accuracy or fidelity can be established. The peak-to-valley (P-V) repeatability errors associated with the experiment, listed in Table 1 , represent approximately 20 percent to 50 percent of the difference between the model and experimental data listed in Table 2. Additionally, the root-mean-square (RMS) repeatability errors, listed in Table 1, represent approximately 4 percent to 8 percent of the difference. However, since the model and experimental difference errors appear to be systematic and spatially slowly varying, reasonable and accurate conclusions can be made. Furthermore, as discussed earlier, material property uncertainties can be on the order of 5 to 10 percent. Other authors have reported difference errors for much smaller scaled models on the order of 8 percent of maximum surface displacement using finite element models. Therefore, the above data strongly supports the verification of the finite element model.

\subsection{Control Model Development}

A control model was developed to investigate static surface shape control utilizing the validated finite element model. This model was used to demonstrate the value of using a validated plant model versus using less accurate models. The National 
Ignition Facility (NIF) adaptive optics configuration was utilized for a specific example. The plant model utilized the output from the finite element model. The sensor model was based on a finite difference matrix representation of a Shack-Hartmann sensor. The controller model utilized a typical least squares control algorithm. With the control model developed, simulations were completed to show the general functionality of the control to correct for typical NIF wavefront aberrations. Lastly, performance comparisons were made between the finite element static plant model and a Gaussian plant model.

\subsection{Performance Comparison}

The control model developed was utilized to compare two types of plant models. The errors associated with using traditional analytical functions, e.g., Gaussian functions, versus the validated finite element model developed in this work, was studied.

With the Gaussian plant model developed, three disturbance cases were solved and compared to the finite element model solution. The three cases included, a piston aberration, a 2nd order aberration, and a 4th order aberration. The piston aberration is probably the most important test case, since the answer is known, i.e., the resulting surface should be perfectly flat. The first comparison was made with the piston aberration correction. The Gaussian plant model produces residual errors approximately 29 times greater than the finite element model results. These results are depicted in Figure 4 and Figure 5. As expected, the Gaussian function produced large errors near the edges, since these analytical functions do not maintain continuity in displacement, slope, and higher derivatives between adjacent influence functions or at edge boundary conditions. Authors have implemented techniques or "tricks" to achieve better boundary condition matches using analytical functions. The Gaussian plant model 2nd order residual error is approximately 6 times greater than the finite element results, and the 4th order residual error is approximately 2 times greater. These results are summarized in Table 3. 


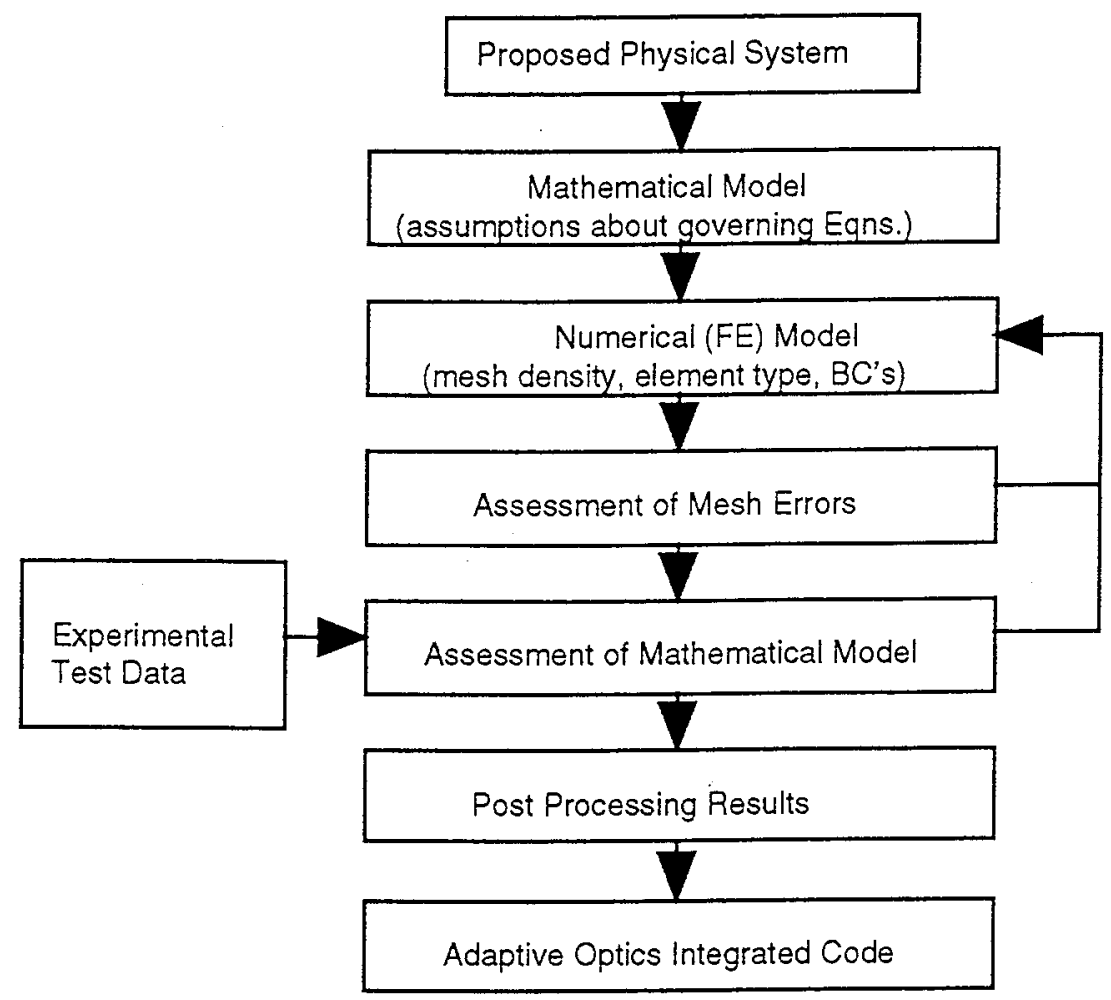

Figure 1. Block diagram of modeling methodology

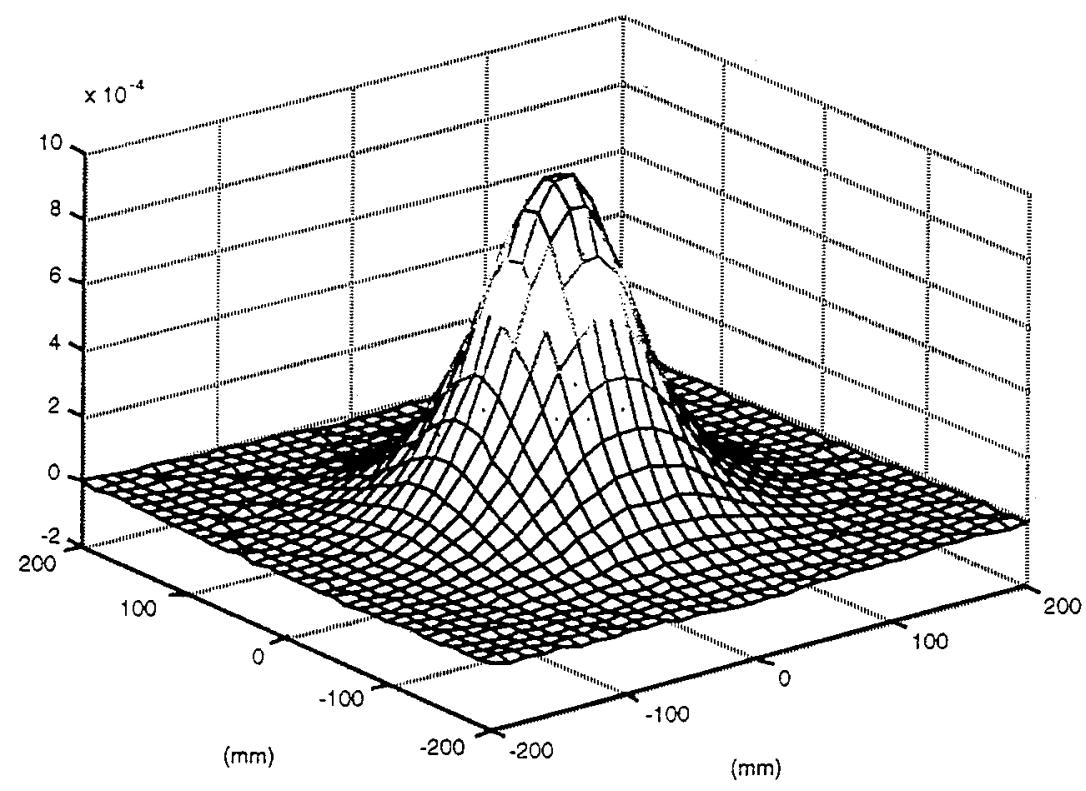

Figure 2. Influence function for center actuator 


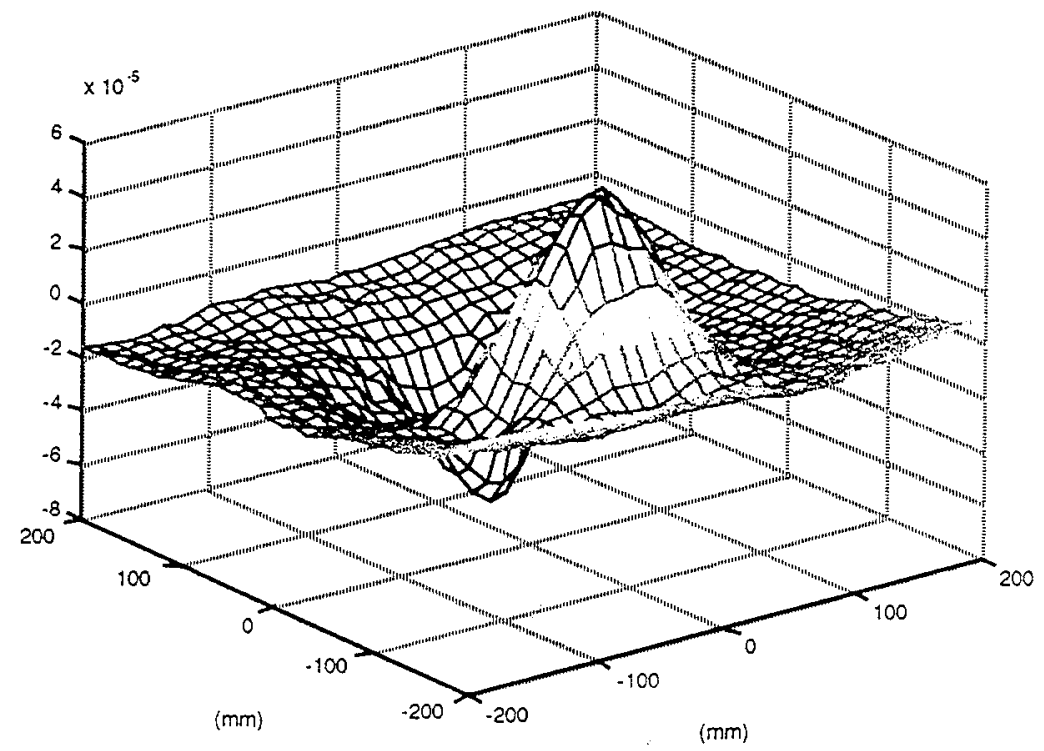

Figure 3. Difference between model and experimental data for the center actuator

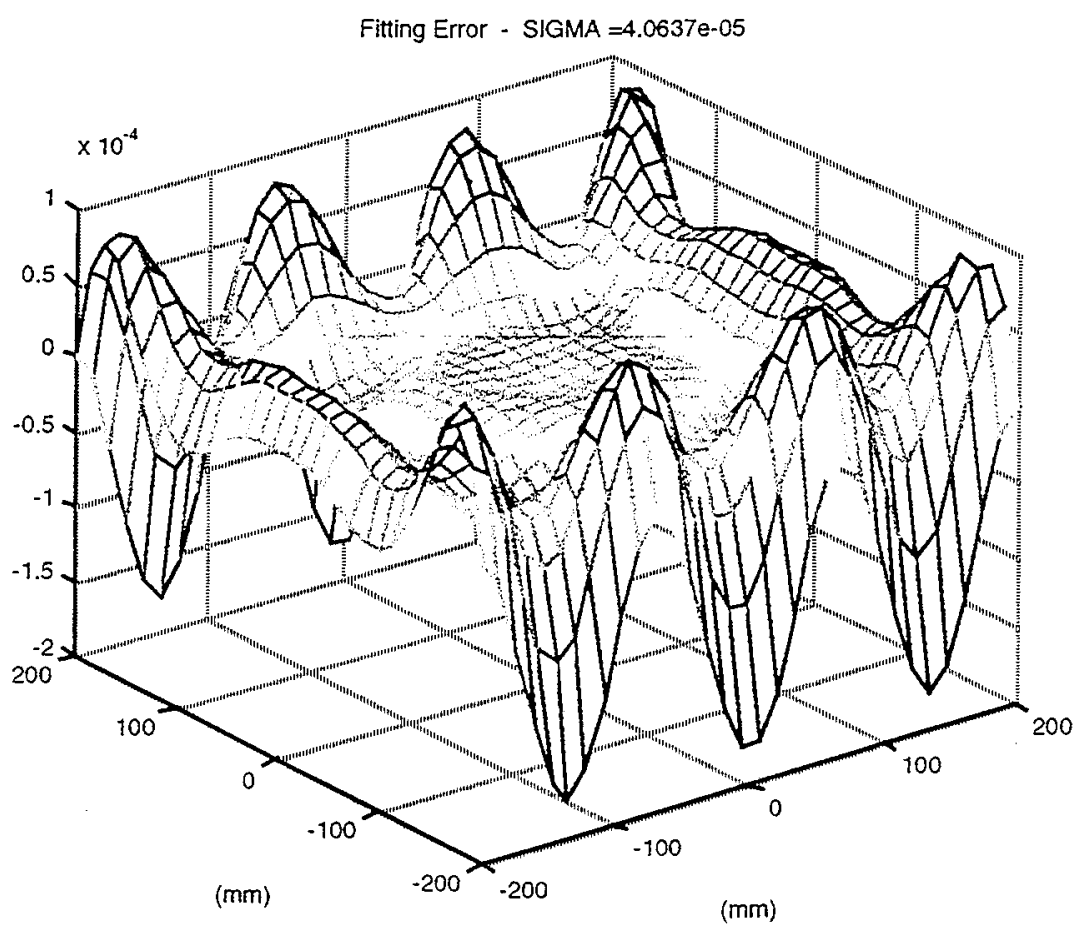

Figure 4. Correction of a piston aberration with Gaussian model 


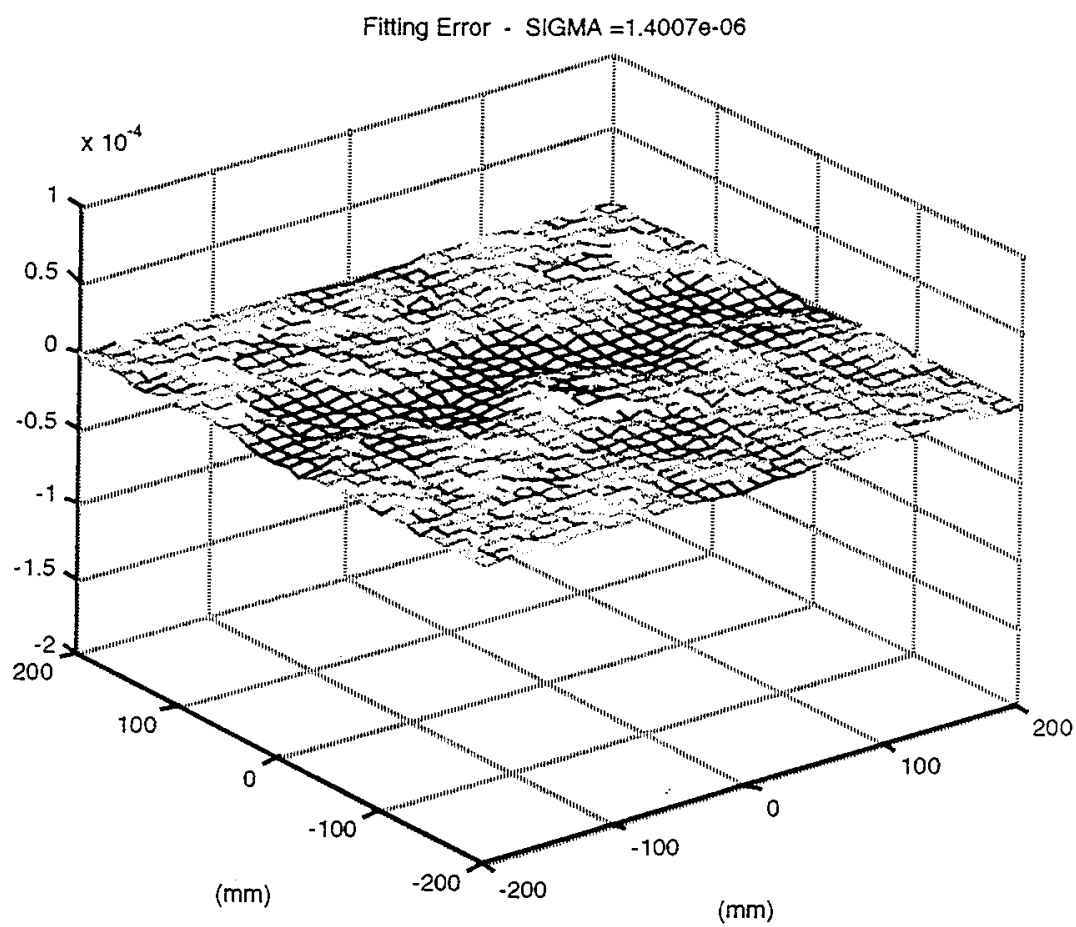

Figure 5. Correction of a piston aberration with FE model

\begin{tabular}{|c|c|c|}
\hline Test & RMS Result & Peak-to-Valley Result \\
\hline Accuracy & $0.034 \mu \mathrm{m}(1.3 \mu \mathrm{in})$ & $0.2 \mu \mathrm{m}(7.9 \mu \mathrm{in})$ \\
\hline Repeatability & $0.005 \mu \mathrm{m}(0.20 \mu \mathrm{in})$ & $0.028 \mu \mathrm{m}(1.1 \mu \mathrm{in})$ \\
\hline Reproducibility & $0.008 \mu \mathrm{m}(0.32 \mu \mathrm{in})$ & $0.057 \mu \mathrm{m}(2.2 \mu \mathrm{in})$ \\
\hline
\end{tabular}

Table 1. Summary of interferometer characterization

\begin{tabular}{|c|c|c|c|}
\hline $\begin{array}{c}\text { Actuator } \\
\text { Number }\end{array}$ & $\begin{array}{c}\text { Maximum } \\
\text { Displacement }(\mu \mathrm{m})\end{array}$ & $\begin{array}{c}\text { Max. Difference } \\
\text { between Experiment } \\
\text { and Model Data }(\mu \mathrm{m})\end{array}$ & $\begin{array}{c}\text { Percent } \\
\text { Difference } \\
(\%)\end{array}$ \\
\hline 3 & 1.0 & 0.06 & $+/-6$ \\
\hline 4 & 1.0 & 0.03 & $+/-3$ \\
\hline 20 & 1.0 & 0.05 & $+/-5$ \\
\hline
\end{tabular}

Table 2. Percent difference between model and experimental data 


\begin{tabular}{|c|c|c|c|c|c|c|}
\hline Aberration & Form & $\begin{array}{c}\text { P-V } \\
\text { displacemen } \\
\mathrm{t}(\mu \mathrm{m})\end{array}$ & $\begin{array}{c}\text { Gaussian } \\
\text { residual } \\
(\mathrm{rms})(\mathrm{nm})\end{array}$ & $\begin{array}{c}\text { Finite Element } \\
\text { residual }(\mathrm{rms}) \\
(\mathrm{nm})\end{array}$ & $\begin{array}{c}\text { Percent } \\
\text { Difference } \\
(\%)\end{array}$ & $\begin{array}{c}\text { Multipl } \\
\text { e Factor }\end{array}$ \\
\hline Piston & $\mathrm{a}$ & 1 & 40.1 & 1.40 & 2800 & 29 \\
\hline 2nd order & $\mathrm{by}^{2}$ & 4 & 150 & 26.9 & 457 & 5.6 \\
\hline 4th order & $\mathrm{cy}^{4}$ & 1 & 65.1 & 27.2 & 140 & 2.4 \\
\hline
\end{tabular}

Table 3. Comparison between Gaussian and finite element plant models 


\section{Chapter 2 - An Adaptive Optic for Correcting Low-Order Wavefront Aberrations}

\begin{tabular}{|c|}
\hline By: Julia Wilhelmsen \\
Charles Thompson \\
Lawrence Livermore National Laboratory \\
August 1999
\end{tabular}




\section{Abstract:}

Adaptive Optics used for correcting low-order wavefront aberrations were tested and compared using interferometry, beam propagation, and a far-field test. Results confirm that the design and manufacturing specifications were met. Expermental data also confirms theoretical performance expectations, indicating the usefulness of these optics (especially in a laser-beam processing system), and identifying the resulting differences between the two fabrication methods used to make the optics. 


\section{Table of Contents}

Introduction

Materials and Methodology

4

Presentation and Discussion of Data 12

Summary and Conclusion $\quad 21$

References $\quad 22$

Appendix 23 


\section{List of Figures}

Figure 1 Translation of optical elements produces astigmatism

Figure 2 Example of photolithography mask used in fabrication

Figure 3 Standard photolithography steps for binary diffractive optics

Figure 4 Set-up for interferometry testing

Figure 5 Set-up for beam propagation and far field testing

Figure 6 Inteferometry image from Phase-plate part with no shear

Figure 7 inteferometry image from Raytheon part with no shear

Figure 8 Inteferometry image from Phase-plate part with $10 \mathrm{~mm}$ shear on the $x$-axis

Figure 9 Inteferometry image from Raytheon part with $10 \mathrm{~mm}$ shear on the $y$-axis

Figure 10 Graph of PV and Residual RMS on x-axis, Raytheon part

Figure 11 Graph of PV and Residual RMS on y-axis, Raytheon part

Figure $12 \mathrm{Sag}$ on the $\mathrm{x}$-axis, Raytheon part

Figure $13 \mathrm{Sag}$ on the $y$-axis, Raytheon part

Figure 14 Graph of PV and Residual RMS on x-axis, Phase-plate part

Figure 15 Graph of PV and Residual RMS on y-axis, Phase-plate part

Figure $16 \mathrm{Sag}$ on the $\mathrm{x}$-axis, Phase-plate part

Figure 17 Sag on the $y$-axis, Phase-plate part

Figure 18 Experimental beam propagation of $-200 \mathrm{~m}$ through center of Raytheon part

Figure 19 Experimental beam propagation of $-200 \mathrm{~m}$ through center of Phaseplate part

Figure 20 Theoretical beam propagation of $-200 \mathrm{~m}$ through center of Raytheon part

Figure 21 Theoretical beam propagation of $-200 \mathrm{~m}$ through center of Phaseplate part

Figure 22 Experimental beam propagation of $-200 \mathrm{~m}$ through corner $(20,-25)$ of Raytheon part

Figure 23 Theoretical beam propagation of $-200 \mathrm{~m}$ through corner $(20,-25)$ of Raytheon part

Figure 24 Far-field image with no optic (Rectangular diffraction pattern)

Figure 25 Far-field image through center of Raytheon part

Figure 26 Far-field image through center of Phase-plate part 


\section{Introduction:}

In this project we characterized two adaptive optics used for correcting low-order wavefront aberrations. In his book Principles of Adaptive Optics, Robert Tyson gives a simple definition, Adaptive optics is "a method of automatically keeping the light focused when it gets out of focus"[1]. An adaptive optic system can be as natural as the human eye, or as technological as the laser guide star system used in astronomy. When it is recognized that a laser is a light source, the simple definition above can encompass even high-power laser systems. Adaptive optics are needed in all of these systems because optical systems are not ideal; aberrations do exist. Aberrations can reduce system efficiency, or prevent a system from fulfilling its design requirements. Deformable mirrors are a widely known example of an adaptive optic.

The adaptive optics used in this project address the correction of primarily astigmatism. Other types of aberrations could include: spherical, coma, chromatic, and distortion. In an astigmatic wavefront two focal planes appear, orthogonal to one another, at different longitudinal points.

Each adaptive optic in this project is comprised of two elements. Both elements are shaped with a cubic curve such that the two elements are complimentary. Intuitively we see that in this position (neutral) the optic is like a plano piece of glass. A collimated wavefront passing through it would not be affected. When the elements are translated
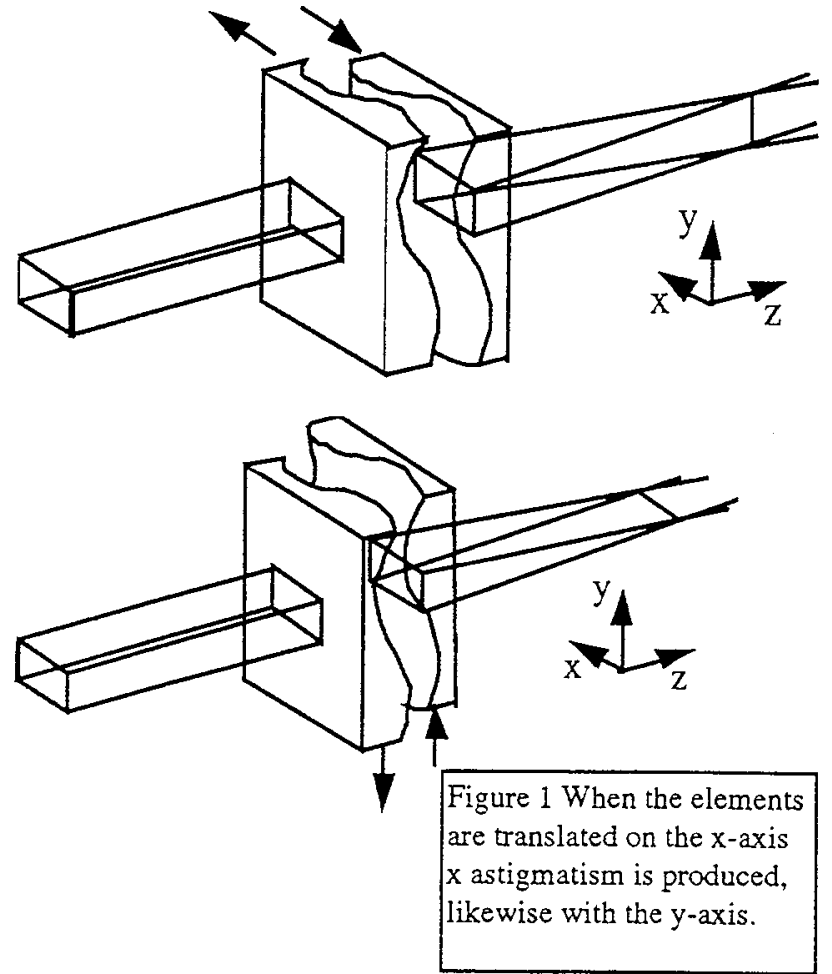
their profiles no longer cancel each other. The result is a quadratic phase variation deriving from the difference of the profiles. The curve used on these optics produced an astigmatic wavefront. Simply put, sliding the elements along $x$-axis produces astigmatism in the $\mathrm{x}$-axis, and likewise sliding the elements on the $y$-axis produces astigmatism in the $y$-axis (Figure 1). The correct curve for this kind of system was developed by Luis Alvarez [2][3][4].

There are different kinds of adaptive optics, each with advantages and disadvantages. A feature of this optic that is both an advantage and a disadvantage is its simplicity. This optic is primarily for correcting astigmatism, other optics such as deformable mirrors can correct higherorder aberrations. The computing power required to run an adaptive optic is directly related to how complex the optic is. It follows that the optic discussed here requires less computing power than many more complicated systems. Another advantage of this more simplistic system is its continuity. A deformable mirror, while 
able to correct more aberrations can only do so discreetly based on the number of actuators used [5]. The disadvantage is the optics lack of ability to correct other aberrations.

An issue that optics must accommodate in high-power laser systems is the effects of thermal energy in the optical transport system. Excess thermal energy can cause the optics of a system to produce more aberrations, which are primarily astigmatic in nature. If this energy cannot dissipate before the system is used again, the resulting aberrations must be corrected using adaptive optics. The adaptive optic presented in this paper can be used to correct large, low-order aberrations leaving smaller higher-order aberrations for more sophisticated optics, like deformable mirrors. In some thermal situations the corrections necessary would be too large for a deformable mirror to correct alone. 


\section{Materials and Methodology:}

\section{Design specifications}

Each optic is composed of two elements, each of which has a cubic profile determined by the following cubic equation:

$$
Z=a \cdot x^{3}-b \cdot x-c \cdot y^{3}+d \cdot y
$$

Where the coefficients are determined to be:

$$
\begin{aligned}
& \text { Plate } 1 \\
& a=1.63399 \cdot 10^{-7} \mathrm{~mm}^{-2} \\
& b=2.5931 \cdot 10^{-4} \\
& c=4.08497 \cdot 10^{-8} \mathrm{~mm}^{-2} \\
& d=1.3346 \cdot 10^{-4} \\
& \text { Plate 2 } \\
& a=1.63399 \cdot 10^{-7} \mathrm{~mm}^{-2} \\
& b=2.5931 \cdot 10^{-4} \\
& c=-4.08497 \cdot 10^{-8} \mathrm{~mm}^{-2} \\
& d=-1.3346 \cdot 10^{-4}
\end{aligned}
$$


Once manufactured plate $\# 2$ is rotated $180^{\circ}$ about the $y$-axis so that the profiles of the two lens will face each other. The a and c coefficients were chosen such that $10 \mathrm{~mm}$ of shift on each plate (in opposite directions) results in a wavefront Optical Path Difference (OPD) of $3 \lambda$, where $\lambda$ is the operating wavelength of $600 \mathrm{~nm}$ with beam size of $40 \times 80$ $\mathrm{mm}$. The resulting OPD is :

$$
\mathrm{OPD}=6 \cdot(\mathrm{n}-1) \cdot\left(\mathrm{a} \cdot \mathrm{x}^{2} \cdot \xi+c \cdot \mathrm{y}^{2} \cdot \eta\right),
$$

where $\mathrm{n}$ is the index of refraction of the plate and $\xi$ and $\eta$ are the plate displacements. Note the independence of OPD on the $b$ and $d$ coefficients. These coefficients were chosen to minimize the amount of material that needed to be removed from the substrate during fabrication of the optic.

This optic was designed for a beam size of $40 \mathrm{~mm}$ by $80 \mathrm{~mm}$, with a total translation of $\pm 25 \mathrm{~mm}$, resulting in the following lens apertures:

Used aperture area: $90 \mathrm{~mm}$ by $130 \mathrm{~mm}$

Area figured: $92 \mathrm{~mm}$ by $132 \mathrm{~mm}$

Edge aperture: $100 \mathrm{~mm}$ by $140 \mathrm{~mm}$ These values indicate that while only $90 \mathrm{~mm}$ by $130 \mathrm{~mm}$ of the optic will actually be used when the elements are fully translated $92 \mathrm{~mm}$ by $132 \mathrm{~mm}$ will have the profile figured on them.

\section{Fabrication}

Due to the non-rotational symmetric profile required in these adaptive optics nontraditional optical fabrication techniques were explored. Two fabrication methods were attempted. Raytheon Optical Systems Inc. used small tool polishing and the Laser Science and Technology group at Lawrence Livermore National Laboratory used photolithography. 


\section{Small-Tool Polishing}

With this method of fabrication a lens with a continuous curve is produced. The fabrication begins with an optical glass that has been polished flat. A small rotating tool passes over the optic grinding out the desired surface profile. One of the challenges in fabricating these optics was the combination of their size (nearly 6 " on a side) and the steep incline of the curve required at the edges. The steep curvature of the optical surface was too great to measure accurately with a conventional interferometer, and the size of the optics prevented several other methods including a commonly used null corrector. Raytheon proposed an alternative method that tested the optics in transmission by immersing them in a near-index matching solution. This allowed a large surface departure to appear smaller to the measuring interferometer, making the metrology possible. A computer took this metrology information and subtracted the equation of the curve to determine how long the polishing tool would have to be kept at each location on the optic to achieve the desired curve on the next polishing cycle. Great care and several polishing runs had to be used to achieve a curve as smooth as desired by the designers. Any curve deviations, such as tooling marks, will introduce higher-order beam aberrations, or diffraction.

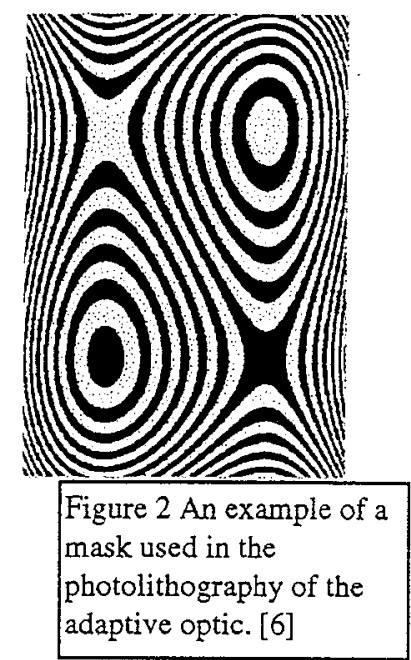

\section{Photolithography}

The photolithography method used by Laser Science and Technology produced 16-level phase-plate optics. In these optics the cubic curve required by the design is not continuous. Instead it is made up of different levels, created using standard multi-level photolithography techniques used in fabricating binary diffractive optics. The first step in the process is to break the profile into discreet phase levels corresponding to $1 \lambda$ path difference, where $\lambda$ is the design wavelength ( $600 \mathrm{~nm}$ in this case).Four masks are fabricated to create this profile on the optic. The first step in the manufacturing process is to apply a photoresist to the substrate. Then the first mask from the previous process (Figure 2) is placed on top of the coated substrate and the optic is exposed to UV

illumination (Figure 3) After the pattern has been exposed onto the photoresist and the photoresist has been chemically developed the part is ready to be chemically etched. The etch rate of fused silica can be controlled so that the silica is removed at a constant pre-determined rate.

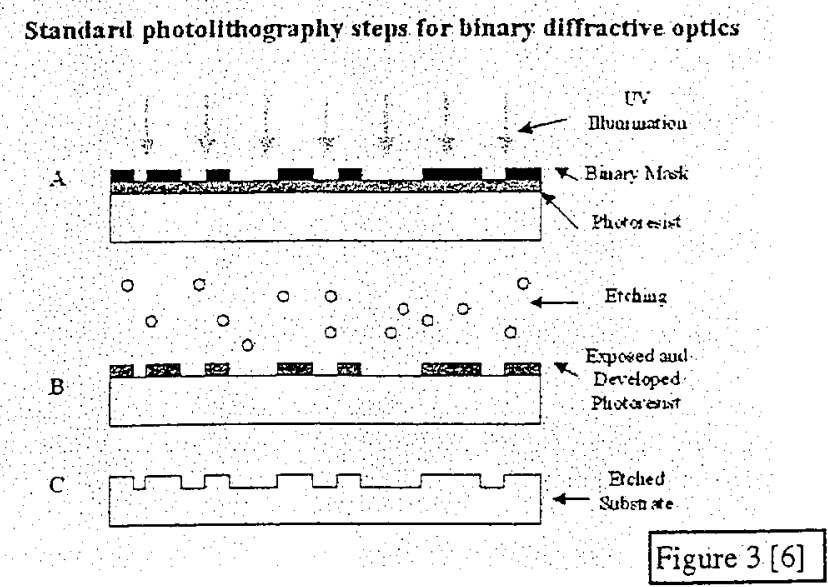


The photoresist prevents the substrate from being etched as deeply as the areas where the photoresist has been developed. To achieve a multi-level phase plate, this process must be repeated. The equation for determining the number of masks required for an $\mathrm{N}$ level phase plate is $2^{\mathrm{n}}=\mathrm{N}$, where $\mathrm{n}$ is the number of masks. It follows that 4 masks are required for each 16 level phase plate for a total of 8 masks for the optic [7]. During fabrication each successive mask must be aligned nearly perfectly to the previous etch. Misalignment results in increased diffraction. When the optic is completed the Anti-Reflection (AR) coating could be applied. The optic as tested, did not have an AR coating.

\section{Tests}

Three tests were run to characterize these optics: Interferometry, Beam Propagation and Far Field. The data collected for all of these tests can be returned as images. In the interferometry test, peak to valley (PV), RMS, and sagitta (sag) numerical values were also tabulated. The set-up for each test is slightly different, but the positioning of the adaptive optic is constant for all three. The set-up for the Raytheon optic has the opposite $y$-axis as the set-up for the Phase-plate because the position of the stepper motor was switched.

\section{Interferometry}

This test is used to analyze the wavefront after the beam passes through the optic. From the measurements we expect to see astigmatic wavefronts caused by the translation of the optical elements. Interferometric data should confirm this

\section{Set-up for Interferometry testing}

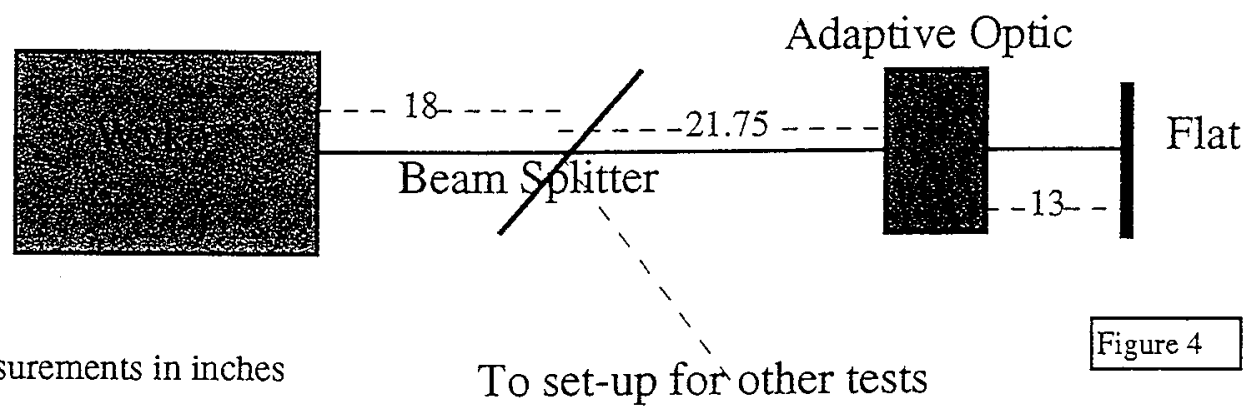

All measurements in inches

To set-up for other tests

along with showing the higher-order residual error due to fabrication. A Wyko 6000

Laser Interferometer was used to measure that distortion.

Figure 4 is the set-up for this test. The beam travels from the Wyko through the optic to a flat. The flat reflects it back through the optic and then back into the Wyko, where the wavefront is measured. The results are displayed on a connected computer using Vision software [8]. The raw data can be exported, as was done in this case, to other programs for further analysis. 


\section{Beam Propagation}

Beam Propagation is used to determine the appearance of the beam at various longitudinal locations. Three positions were used for each measurement: a re-imaged lens plane, and a forward and backward propagation approximately equivalent to $200 \mathrm{~m}$ in free space. The images generated by this test indicate the intensity uniformity at various positions on the beam, with the ideal being uniform.

The set-up for Beam Propagation is in Figure 5. The Wyko was used to provide the collimated laser source. After passing through the optic twice the beam hits a beam splitter which reflects some of the beam through an optical system, which includes a telescope. The end point of this system is a CCD camera, mounted on a metal rail. The various positions required for the three measurements were achieved by moving the camera along the rail. A frame

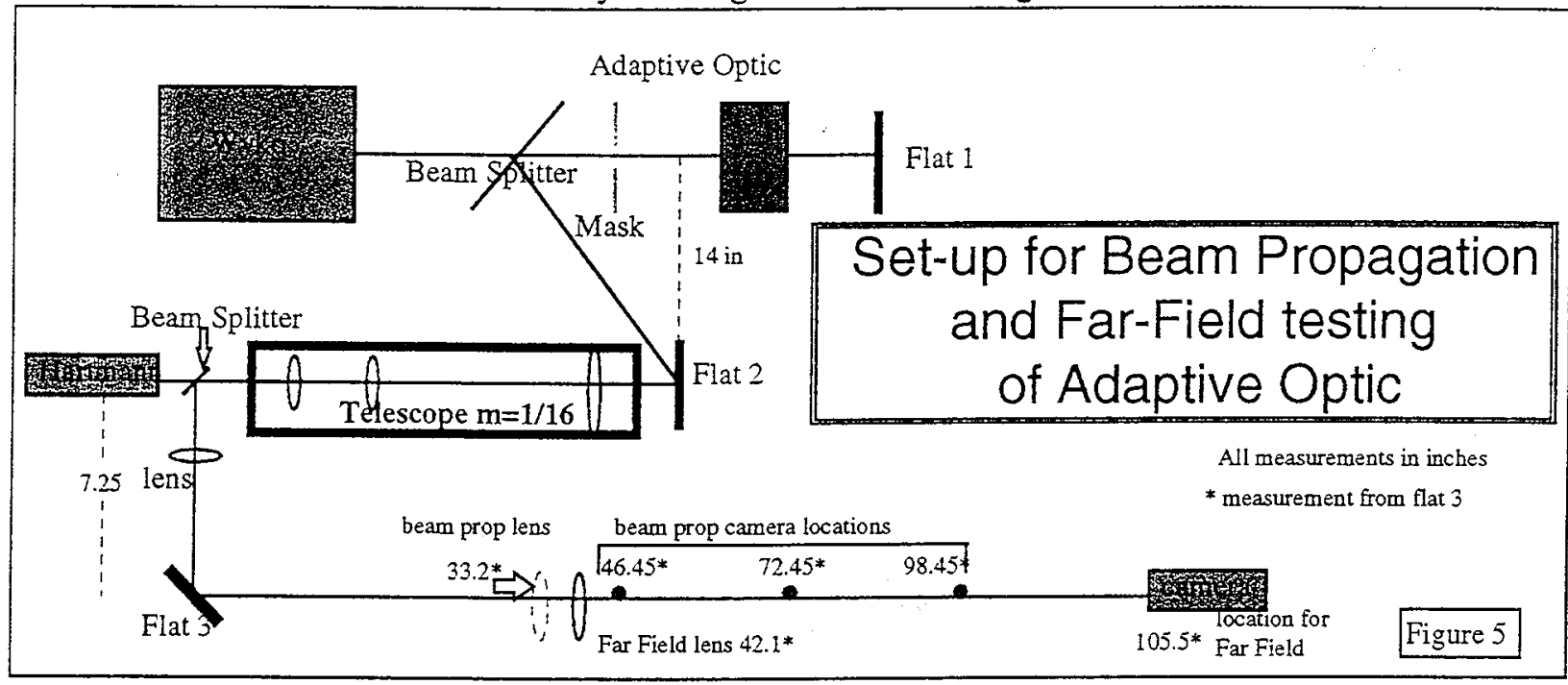

grabber was used to capture the images for this test.

\section{Far-field}

The far field test measures the beam intensity profile at a propagation distance equivalent to infinity. The set-up for this test is very similar to beam propagation. The mirror indicated and the camera are moved such that the image of the beam front is focused onto the camera (Figure 5). This test is useful in determining the effects of diffraction from the optical surface. The tooling marks on Raytheon optics and the phase plate edges contribute to diffraction effects. It is important to minimize those effects. The program used to collect these images was Scion Image [9]. 


\title{
Presentation and Discussion of Data:
}

\author{
Introduction
}

Due to the volume of data collected, only a selection from each test is presented in this section. Some further data can be found in the appendix, including the excel worksheets used to generate the graphs of this section. Unfortunately the large number of images taken from the three tests prevents more than the sampling shown here from being included. The generalizations presented here apply to all data, shown or not.

\section{Interferometry}

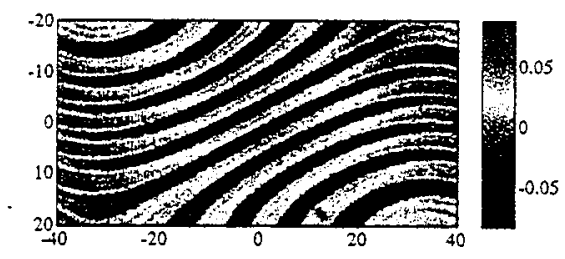

Figure 6 Interferometry image from Phase-plate part with no shear (neutral position).
The wavefront images generated by this test must be processed to obtain numerical data, but alone they still contribute to our understanding of the optics. In the ideal situation optics in their neutral position would produce an unaffected wavefront. Figure 6 is the wavefront

image of the Phase-plate part in its neutral position. The lines in the image are caused by the profile of the optic. These lines will most likely cause the diffraction effects we see in later tests. The Raytheon part is not without its own imperfections. The image from its neutral position (Figure 7), while flatter than the image from the Phase-plate part, has an almost circular pattern. This pattern is most likely caused by tooling marks from fabrication. Attempts were made to minimize the tooling marks. The resulting figure is within specified tolerance.

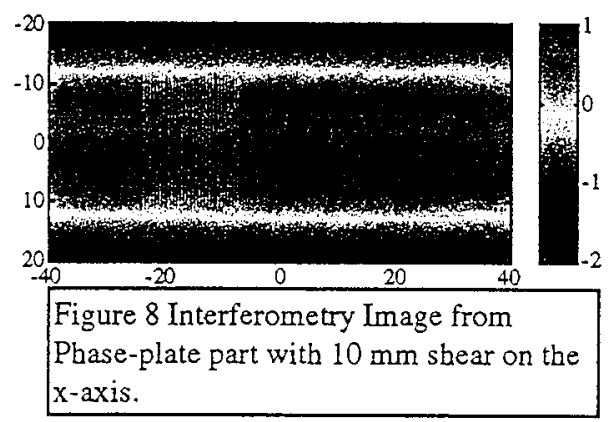

When the optical elements are translated, the astigmatism produced is apparent in the images of the wavefront. When the elements are shifted on the $\mathrm{x}$-axis a horizontal pattern is produced(Figure 8 ), indicating astigmatism in the $\mathrm{x}$-axis, as predicted. The same holds true of shifts along the $y-$ axis(Figure 9). In the phase-plate images

(Figure $\mathrm{X}$ ) the profile of the optics seen at no shear are still evident. These profile lines will most likely cause diffraction effects. They also blur the edges of the pattern shown and contribute to the error in the PV, RMS and sag readings. The lines themselves can be partly attributed to

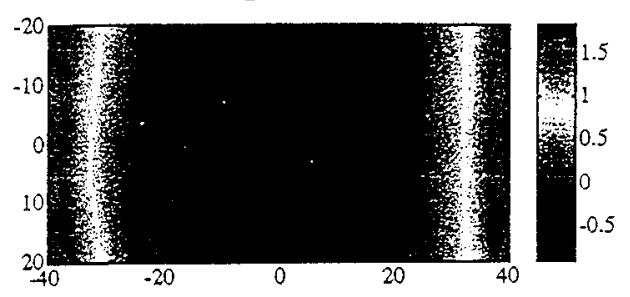

Figure 9 Interferometry Image from Raytheon part with $10 \mathrm{~mm}$ shear on the $y$ axis. 
the wavelength of the laser used for testing. The optics were designed for a $600-\mathrm{nm}$ laser and a 633-nm laser was used.

The numerical data from this test can be summarized in four graphs for each optic. Each measurement produced an image of the wavefront, from which Peak to Valley (PV) and RMS values are calculated. Using the Vision software [8] to remove Tilt, Power and Astigmatism we can find the residual data for PV and RMS. The difference between PV and sag is the residual error of the optics.

The graphs of PV and Residual RMS compare the experimental PV (at $600 \mathrm{~nm}$ ), with the ideal based on the design specifications. The equation to determine the ideal PV or sag is: $\lambda=0.3 \cdot S$, where $\lambda$ is the number of waves and $S$ is the shear on either axis. To calculate the ideal value for the translations off axis (shear in both $\mathrm{x}$ and $\mathrm{y}$ ) calculate the waves for each axis and add them together. The experimental measurements are accurate, however, they do not show exactly was of importance(Figure 10,11,14,15). To examine this more closely a sag measurements were taken. These measurements will more accurately represent the performance of the optic. The sag measurements were performed by fitting a second order polynomial to the interferometric data. The coefficients of the fit were used to determine sag over a given beam width. This information (Figure $X$ ) confirms expectations of performance. The sag measurements for the Raytheon optic have opposite slope from those for the Phase-plate optic because of the difference in optical placement described in the Methodology section.

Another interesting development can be deduced form the PV graph for the Raytheon optic for displacement along the $y$-axis (Figure 11). The PV graph appears shifted to the right of the ideal. Closer examination of the vertex of the graph indicates that the zero on the $y$-axis is not at the center of the optic, but rather at $0.5 \mathrm{~mm}$ in the positive y-direction. Further readings were taken assuming the zero at this new position.

\section{Measurements from Raytheon Optic}

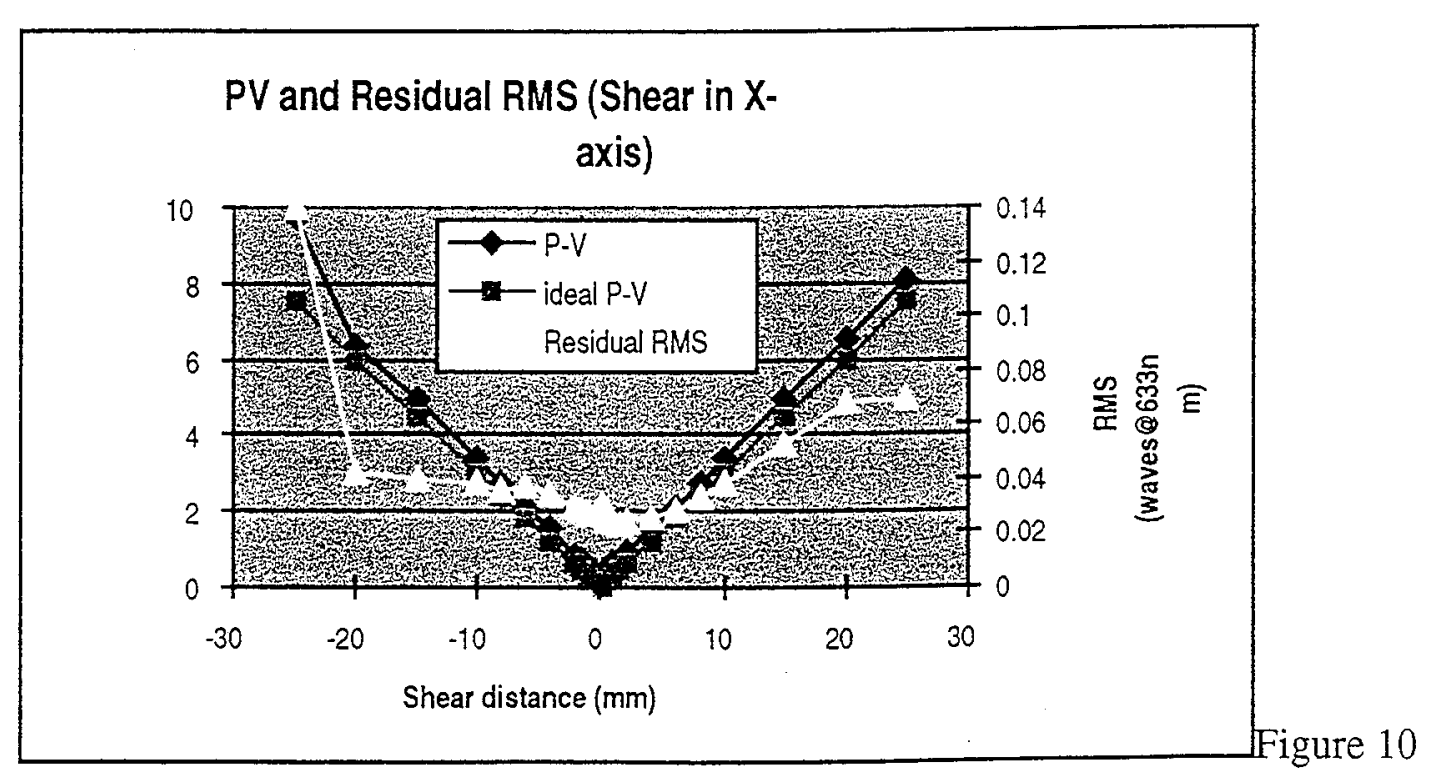




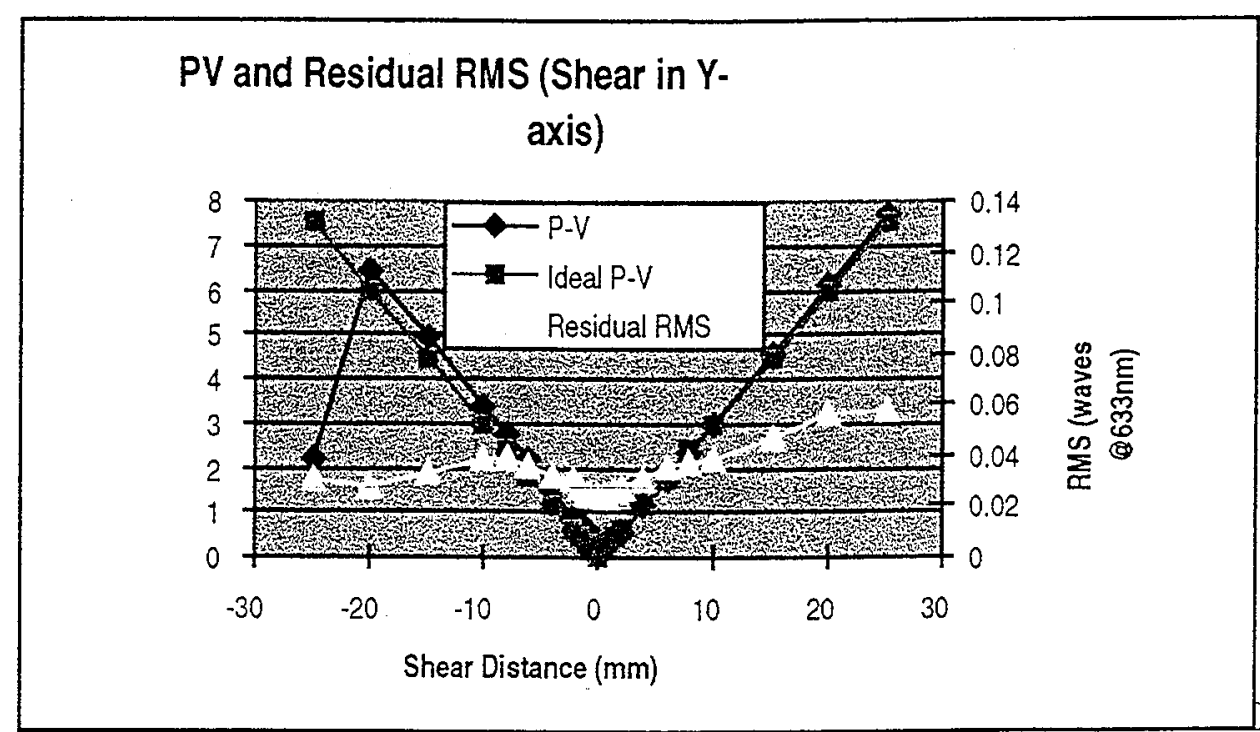

Figure 11

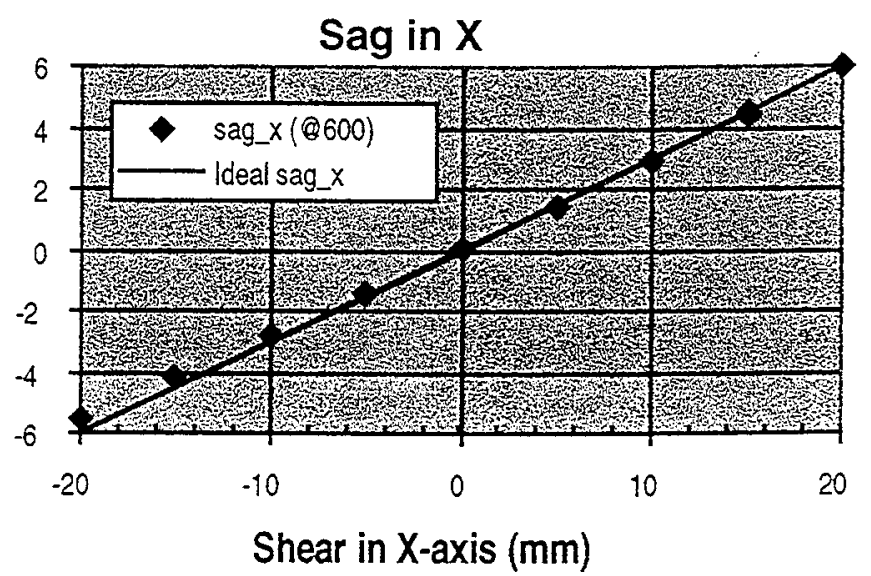

Figure 12

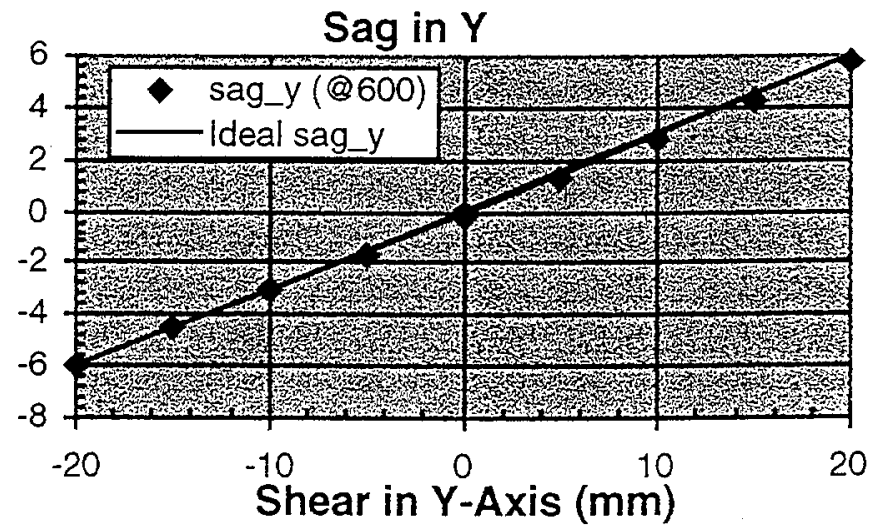

Figure 13 


\section{PV and Residual RMS (Shear in X-Axis)}

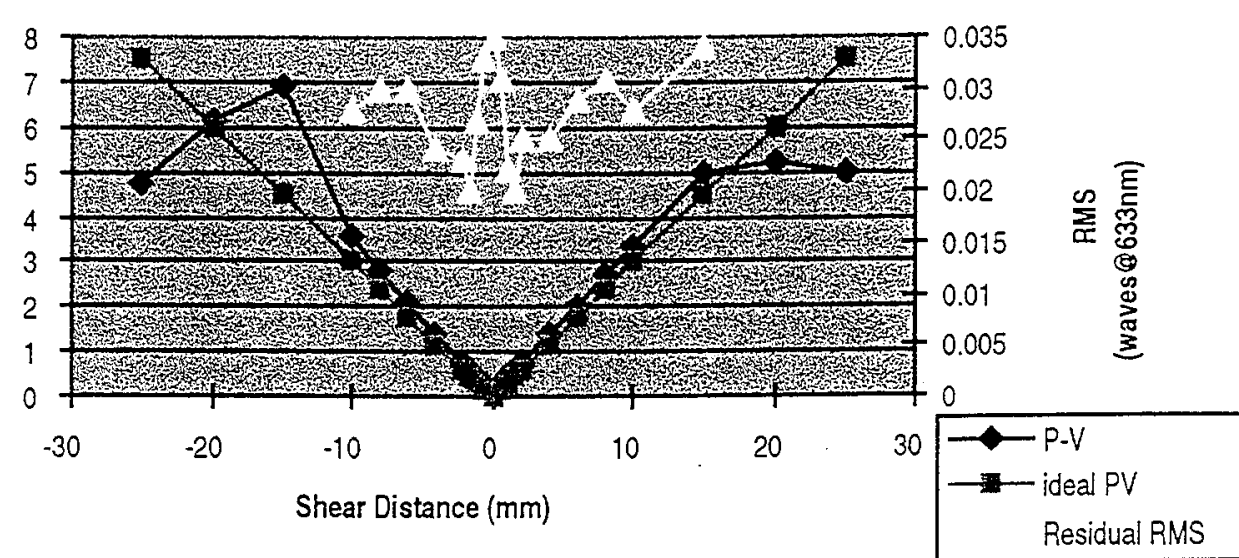

\section{PV and Residual RMS (Shear in Y-axis)}

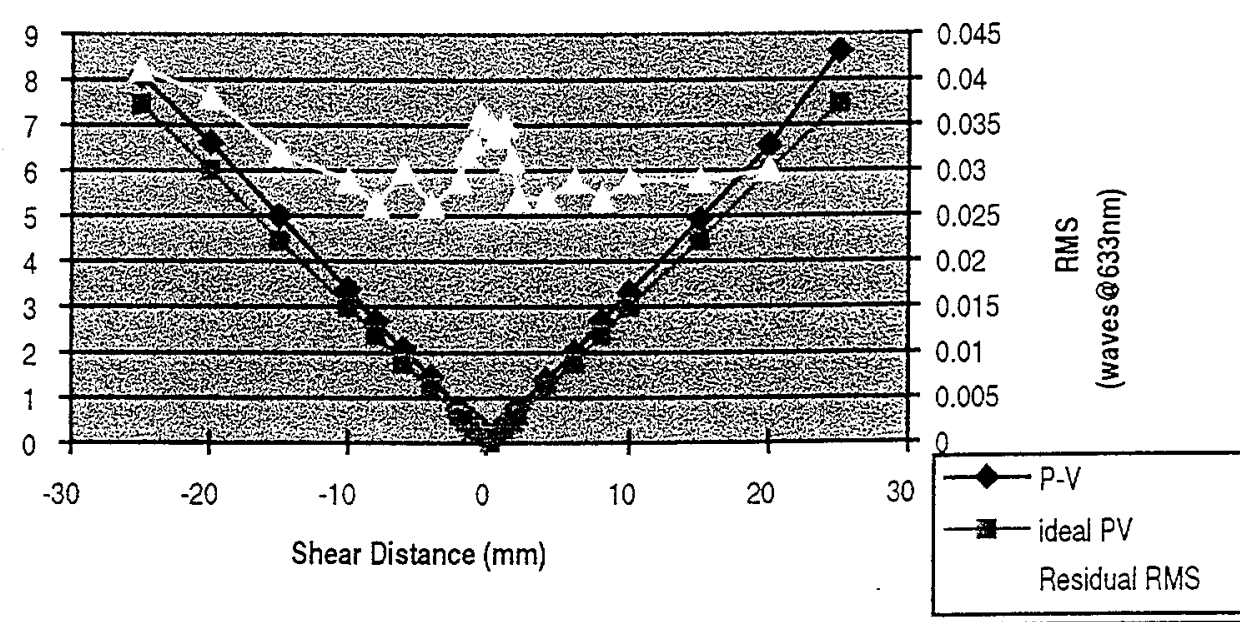

Figure 15 


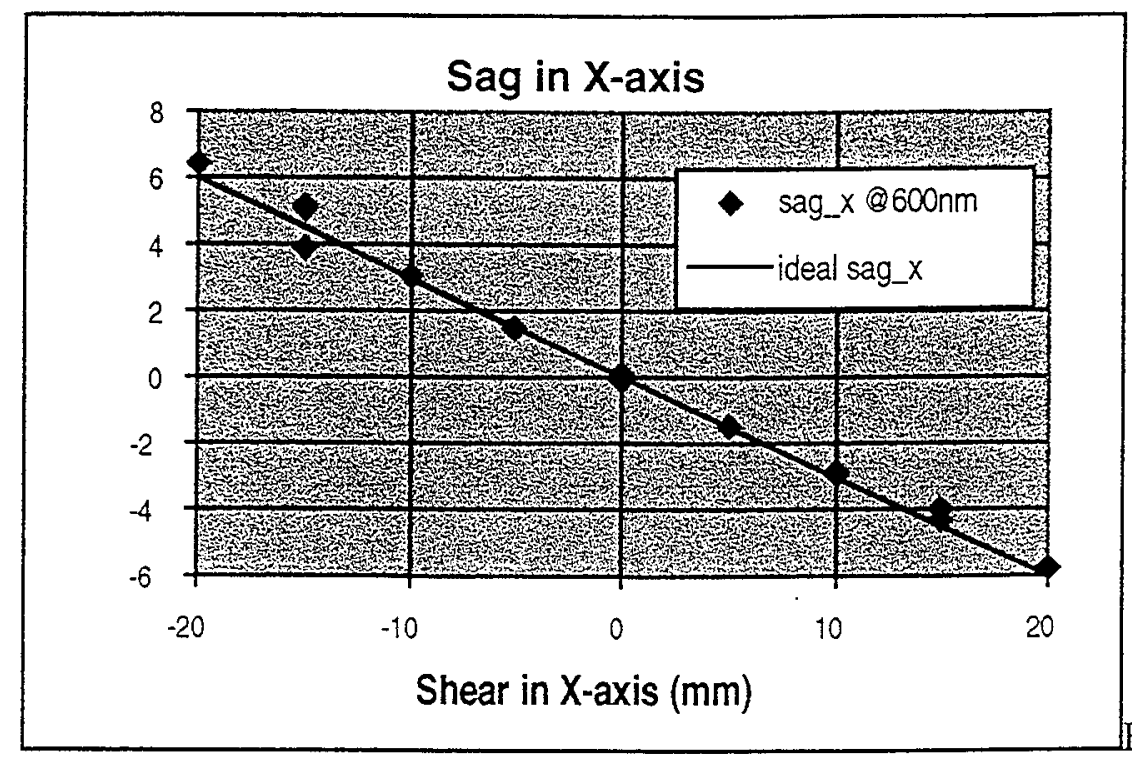

Figure 16

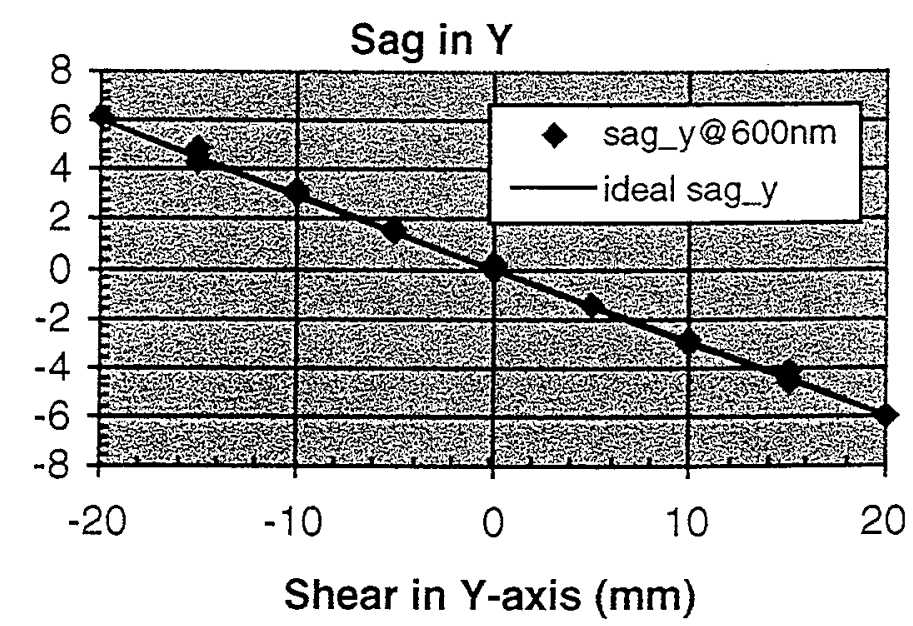

Figure 17

Because the impact of this new "zero" point did not produce significant changes when the optics are translated with relationship to each other, the interferometer data was not retaken. The new zero was used for the beam propagation test.

The data points generated with higher shear values do not appear as accurate as those closer to the neutral position. The Wyko is limited by the slope of the wavefront. When the wavefront has a high slope value the Wyko is unable to get an accurate reading. Greater shear on the optics produces more astigmatism and therefore more slope on the wavefront. These high-shear data points may be inaccurate as a result of the limitations of the Wyko rather than the optic itself. Also contributing may be the edge of the optics and the mounting devices. These would increase diffraction effects should the wavefront come in contact with them during the test. It is also possible that the quality of the optic is better at the center than at the edges. These two features would only minimally affect the readings in comparison to the limitations of the Wyko. 


\section{Beam Propagation}

Beam propagation is used to test the beam intensity uniformity at several longitudinal positions relating to a relayed image of the optic. Ideally when the optic is in its neutral position the beam should have uniform intensity. The Igor program[10] was used to analyze the captured images from beam propagation. It calculated the percent of the image falling into each of two categories: greater than twice the mean intensity and less than half the mean intensity. This is a quantifiable measurement of beam uniformity. A comparison of the beam uniformity from the Raytheon optics and the Phase-plate optics is also useful. The beam was propagated through the center of each optic in the neutral position, and the four corners (still in the neutral position). Using the corresponding Wyko information a theoretical beam propagation was done for comparison with experimental data.

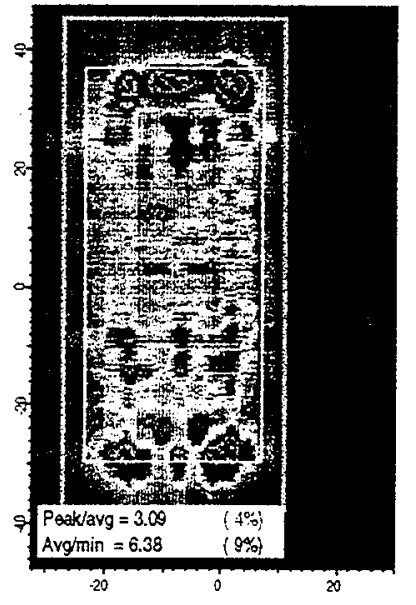

Figure 18 Experimental beam propagation of $-200 \mathrm{~m}$ through center of Raytheon optic.

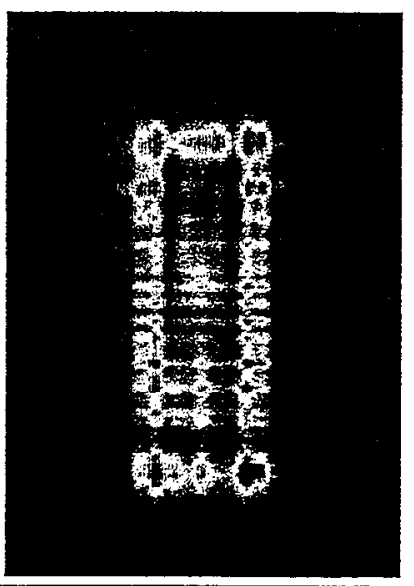

Figure 20 Theoretical beam propagation of $-200 \mathrm{~m}$ through Raytheon optic.
General information about the optics can be deduced by examining the propagation through the center of each optic (Figure 18, 19). The corresponding theoretical propagation (Figure 20,21) agrees well with the data collected. The Phase-plate image is much wider, and more segmented than the Raytheon image indicating diffraction effects as evidenced in the other tests. As in interferometry images, the lines of the Phase-plate are visible due to the diffraction of the beam. A more numerical examination of the intensity can be accomplished by examining the numerical data at the bottom of the image. The percentage in red (top) is the percent of the area of the beam that is greater than twice the mean intensity of the beam. The percentage in blue (bottom) is the percent of the area of the beam that is less than half the mean intensity of the beam. More generally the top number indicates the percentage of the beam much more intense, and the bottom number much less intense, than the mean intensity. A uniform beam (for example with no

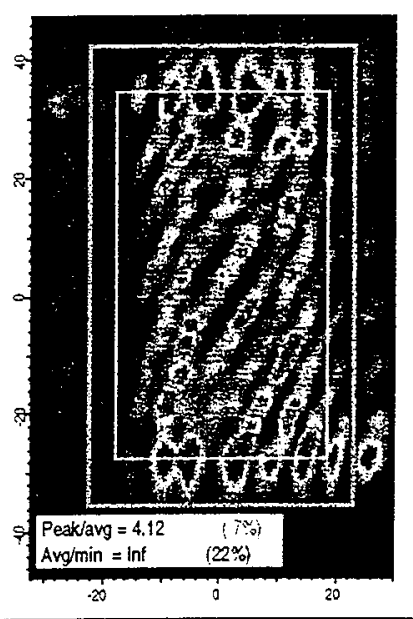

Figure 19 Experimental beam propagation of $-200 \mathrm{~m}$ through center of Phase-plate optic.

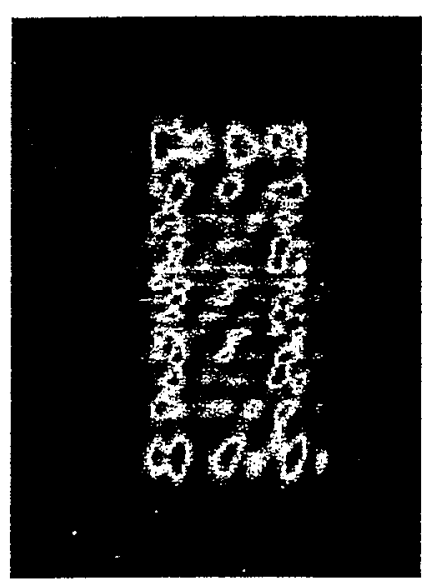

Figure 21 Theoretical beam propagation of $-200 \mathrm{~m}$ through Phase-plate optic.

optic) would indicate zero percent of the beam being more intense or less intense than the mean. Clearly the Raytheon beam is more uniform than the Phase-plate beam. The two 
figures shown are at an equivalent propagation of $-200 \mathrm{~m}$ from a relayed image of the optic, but the data collected from the other two longitudinal positions show similar information.

Another interesting feature pointed out by the beam propagation test is the quality of the corners of the optic. Figure 22 is the experimental beam propagation through the corner corresponding to a shift (in both optics ) of ( $20 \mathrm{~mm},-25 \mathrm{~mm})$. In this position there is no optical power just a shift in the optical axis. The image appears to be skewed to one side. To further examine this feature, the theoretical beam propagation was calculated (Figure 23). The skew in the image is less obvious, but still present, revealing that further aberrations are introduced when the edges of the optic are used. The center of the optic is of higher quality than the edges. This further confirms the seemingly
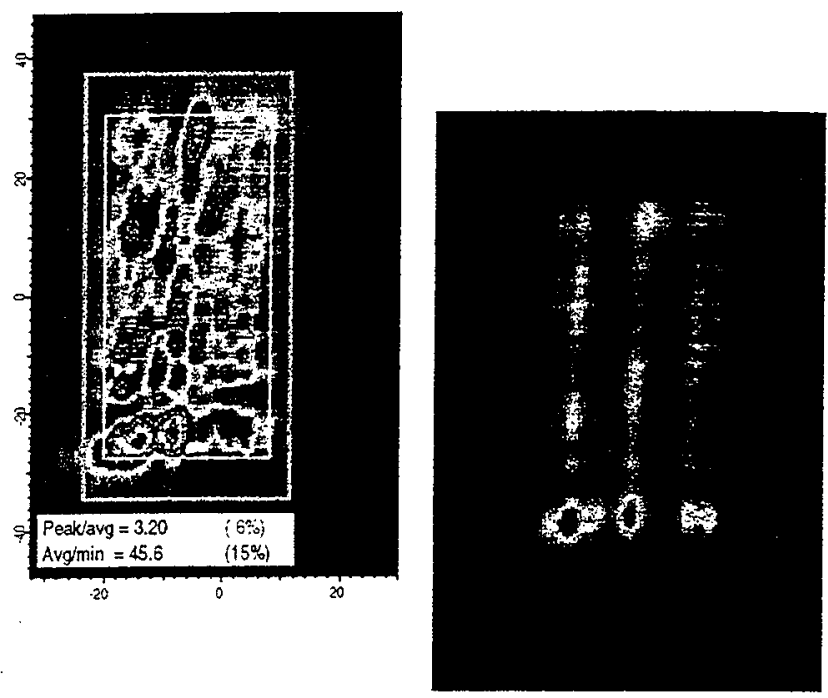

Figure 22 Experimental beam propagation of $-200 \mathrm{~m}$ through corner $(20,-25)$ of Raytheon optic. Notice the slight skew of the image.
Figure 23 Theoretical beam propagation of $-200 \mathrm{~m}$ through corner $(20,-25)$ compare skew with figure to left. misplaced data points in the interferometry data graphs.

\section{Far Field}

No numerical data has been calculated from the farfield test, however a comparison of the far-field images

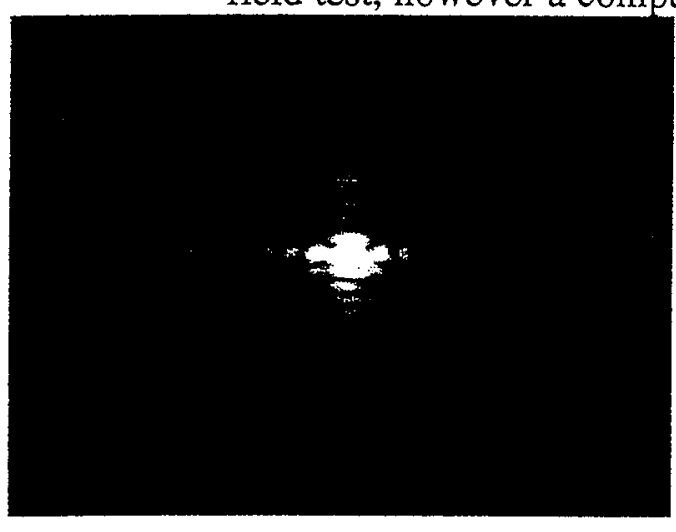

Figure 25 Far-Field image through center of Raytheon optic. Notice the rectangular aperture diffraction pattern.

from the Raytheon parts and the Phase-plate parts can confirm the suggestion from the other tests that there are significant diffraction effects in the Phase-plate optics. A rectangular aperture was used in this test, meaning that if no optic is in place the system should return the diffraction

\section{pattern of a}

rectangular aperture (Figure 24). Ideally when the adaptive optics are in their neutral position they should not affect the diffraction pattern. Figure 25 is the Raytheon-part far-field image with the optics in the neutral position. The image is just as expected, indicating very little diffraction is occurring in the optic, only through the aperture. The phase-plate

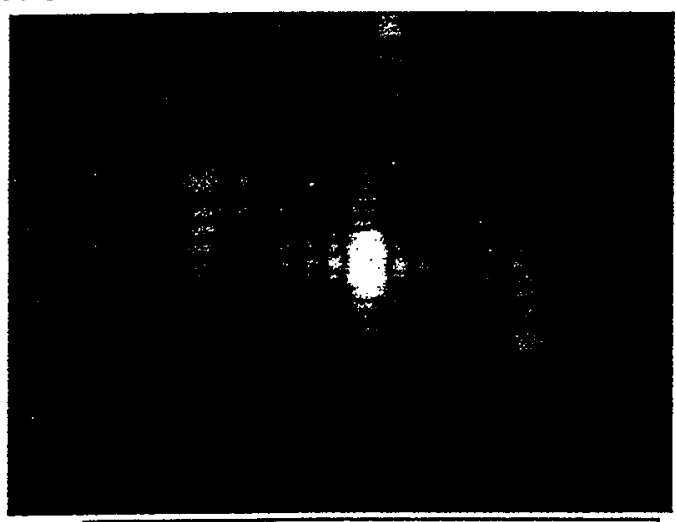

Figure 26 Far-field image through center of phase plate optic. Notice the difference between this Figure and Figure 25. 
far-field image (Figure 26) shows distinct differences from the diffraction pattern of a rectangular-aperture, indicating diffraction in the optic itself. Some of the diffraction of the Phase-plate optic can be attributed to the $633 \mathrm{~nm}$ wavelength it was tested at, versus the design at $600 \mathrm{~nm}$. Other causes of diffraction are binary mask misalignments. Farfield images through the four corners of each optic were also taken. The image of Raytheon parts is not as clean as through the center, however the difference between the Raytheon image and the corresponding phase-plate image is still significant. This again confirms the higher quality of the center of the optic. 


\section{Summary and Conclusion:}

Two adaptive optics were characterized using interferometry, beam propagation and far field tests to determine theoretical versus experimental performance and to compare the two methods of fabrication used to produce the optics.

The optics performed as required, correcting/creating low-order astigmatic wavefronts. Tests also reveal that the center of both optics is of higher quality than the edges. The PV, RMS and sag measurements conform closely to expected values, except close to these edges.

The performance of both optics confirms the Raytheon parts were fabricated to a tighter tolerance, and therefore, the Raytheon PV, RMS and sag measurements conform more closely to expected values than the Phase-plate values. The Raytheon wavefront also had more uniform beam intensity and less diffraction, and is usable over a great band of wavelengths. Despite the better performance of the Raytheon parts, the Phaseplate optic may still be effective for some uses, at significant financial benefit, particularly in a laser system that can tolerate scatter losses.

The adaptive optical elements detailed within this report show great promise and future uses should be investigated, especially in the areas of beam processing. Using the

optics in conjunction with higher-order correctors to induce larger corrections than could be achieved singly should also be considered. 


\section{References}

[1] R. K. Tyson Principles of Adaptive Optics, Second Edition, p. 3, Academic Press, San Diego, 1998.

[2] L.W. Alvarez, 'Two-element variable-power spherical lens,' U.S. Patent \#3305294 (1967).

[3] L.W. Alvarez and W. E. Humphrey, 'Variable-power lens and system,' U.S. Patent \#3507565 (1970).

[4]L.W. Alvarez, 'Development of variable-focus lenses and a new refractor,' J. Am. Optom. Assoc. 49 (1) p. $24-29$ (1978).

[5] R. K. Tyson Principles of Adaptive Optics, Second Edition, p. 210-219, Academic Press, San Diego, 1998.

[6] Image courtesy of Ian Barton

[7] I.M. Barton, S.N. Dixit, L.J. Summers, C.Thompson, and K.Avicola, 'A diffractive Alvarez lens, to be submitted to Optics letters, 1999.

[8] Wyko Vision for optical testing, Wyko Corp., version 1.800 Copyright 1996.

[9] Scion Image, an adaptation of $\mathrm{NiH}$ image by Wayne Rasband, National Institute of Health.

[10] Igor Pro, WaveMetrics Inc., version 3.13, copyright 1998-1998 


\section{Appendix}

Interferometry data for Raytheon optic

Coordinates in table are for one element the other element would be moved just the opposite.

\begin{tabular}{|c|c|c|c|c|c|}
\hline $\begin{array}{l}\text { Axis } \\
(\mathrm{mm})\end{array}$ & $\begin{array}{l}\text { Y Axis } \\
(\mathrm{mm})\end{array}$ & $P-V(633)$ & P-V $(600 n m)$ & $\begin{array}{l}\text { Ideal } \\
V\end{array}$ & Residual RMS \\
\hline 0 & -25 & 2.116 & 2.23238 & 7.5 & 0.03 \\
\hline 0 & -20 & 6.118 & 6.45449 & 6 & 0.027 \\
\hline 0 & -15 & 4.649 & 4.904695 & 4.5 & 0.033 \\
\hline 0 & -10 & 3.181 & 3.355955 & 3 & 0.039 \\
\hline 0 & -8 & 2.607 & 2.750385 & 2.4 & 0.038 \\
\hline 0 & -6 & 2.051 & 2.163805 & 1.8 & 0.035 \\
\hline 0 & -4 & 1.443 & 1.522365 & 1.2 & 0.032 \\
\hline 0 & -2 & 0.839 & 0.885145 & 0.6 & 0.03 \\
\hline 0 & -1.5 & 0.712 & 0.75116 & 0.45 & 0.027 \\
\hline 0 & -1 & 0.561 & 0.591855 & 0.3 & 0.025 \\
\hline 0 & -0.5 & 0.429 & 0.452595 & 0.15 & 0.025 \\
\hline 이 & 0 & 0.289 & 0.304895 & 0 & 0.025 \\
\hline of & 0.5 & 0.208 & 0.21944 & 0.15 & 0.025 \\
\hline of & 1 & 0.305 & 0.321775 & 0.3 & 0.024 \\
\hline 0 & 1.5 & 0.422 & 0.44521 & 0.45 & 0.026 \\
\hline 0 & 2 & 0.548 & 0.57814 & 0.6 & 0.027 \\
\hline 0 & 4 & 1.092 & 1.15206 & 1.2 & 0.03 \\
\hline 0 & 6] & 1.651 & 1.741805 & 1.8 & 0.035 \\
\hline 0 & 8 & 2.219 & 2.341045 & 2.4 & 0.037 \\
\hline 0 & 10 & 2.848 & 3.00464 & 3 & 0.039 \\
\hline 0 & 15 & 4.339 & 4.577645 & 4.5 & 0.047 \\
\hline 0 & 20 & 5.807 & 6.126385 & 6 & 0.056 \\
\hline 0 & 25 & 7.302 & 7.70361 & 7.5 & 0.057 \\
\hline 25 & 0 & 7.632 & 8.05176 & 7.5 & 0.07 \\
\hline 20 & 0 & 6.208 & 6.54944 & 6 & 0.068 \\
\hline 15 & 인 & 4.735 & 4.995425 & 4.5 & 0.053 \\
\hline 10 & 0 & 3.194 & 3.36967 & 3 & 0.039 \\
\hline 8 & 0 & 2.636 & 2.78098 & 2.4 & 0.033 \\
\hline 6 & of & 2.023 & 2.134265 & 1.8 & 0.028 \\
\hline 4 & 0 & 1.47 & 1.55085 & 1.2 & 0.026 \\
\hline 2 & 이 & 0.876 & 0.92418 & 0.6 & 0.024 \\
\hline 1.5 & 0 & 0.716 & 0.75538 & 0.45 & 0.023 \\
\hline 1 & 0 & 0.579 & 0.610845 & 0.3 & 0.025 \\
\hline 0.5 & 0 & 0.434 & 0.45787 & 0.15 & 0.025 \\
\hline 0 & 0 & 0.304 & 0.32072 & 0 & 0.031 \\
\hline-0.5 & 0 & 0.352 & 0.37136 & 0.15 & 0.027 \\
\hline-1 & 0 & 0.54 & 0.5697 & 0.3 & 0.027 \\
\hline-1.5 & of & 0.676 & 0.71318 & 0.45 & 0.028 \\
\hline-2 & 0 & 0.826 & 0.87143 & 0.6 & 0.03 \\
\hline-4 & 0 & 1.437 & 1.516035 & 1.2 & 0.035 \\
\hline-6 & o & 2.028 & 2.13954 & 1.8 & 0.037 \\
\hline-8 & 0 & 2.629 & 2.773595 & 2.4 & 0.036 \\
\hline-10 & 0 & 3.225 & 3.402375 & 3 & 0.039 \\
\hline-15 & 0 & 4.672 & 4.92896 & 4.5 & 0.04 \\
\hline
\end{tabular}




\begin{tabular}{|c|c|c|c|c|c|}
\hline-20 & 이 & 6.036 & 6.36798 & 6 & 0.042 \\
\hline-25 & 0 & 9.246 & 9.75453 & 7.5 & 0.14 \\
\hline 1 & 1 & 0.591 & 0.623505 & 0.6 & 0.025 \\
\hline-1 & 1 & 0.466 & 0.49163 & 0.6 & 0.028 \\
\hline-1 & -1 & 0.78 & 0.8229 & 0.6 & 0.029 \\
\hline 1 & -1 & 0.79 & 0.83345 & 0.6 & 0.026 \\
\hline 5 & 5 & 2.8 & 2.954 & 3 & 0.034 \\
\hline-5 & 5 & 2.751 & 2.902305 & 3 & 0.035 \\
\hline-5 & -5 & 3.17 & 3.34435 & 3 & 0.04 \\
\hline 5 & -5 & 3.083 & 3.252565 & 3 & 0.031 \\
\hline 10 & 10 & 5.752 & 6.06836 & 6 & 0.053 \\
\hline-10 & 10 & 5.591 & 5.898505 & 6 & 0.21 \\
\hline-10 & -10 & 6.04 & 6.3722 & 6 & 0.044 \\
\hline 10 & -10 & 5.937 & 6.263535 & 6 & 0.04 \\
\hline 15 & 15 & 8.887 & 9.375785 & 9 & 0.054 \\
\hline-15 & 15 & 8.435 & 8.898925 & 9 & 0.032 \\
\hline-15 & -15 & 9.016 & 9.51188 & 9 & 0.042 \\
\hline 15 & -15 & 8.815 & 9.299825 & 9 & 0.041 \\
\hline \multicolumn{6}{|c|}{ Using .5 in $Y$ as zero } \\
\hline 0 & 10 & 2.825 & 2.980375 & 3 & 0.041 \\
\hline 0 & -10 & 3.078 & 3.24729 & 3 & 0.039 \\
\hline
\end{tabular}




\section{Sag measurements for Raytheon Optic}

\begin{tabular}{|c|c|c|c|c|c|c|c|}
\hline$x$ & $y$ & $\operatorname{sag} x$ & sag_y & $\begin{array}{l}\operatorname{sag}_{(@ 600)} x \\
(@)\end{array}$ & $\begin{array}{l}\text { sag_y } \\
(@ 600)\end{array}$ & \begin{tabular}{|l|} 
deal \\
sag_ $x$
\end{tabular} & $\begin{array}{l}\text { Ideal } \\
\text { sag_y }\end{array}$ \\
\hline 0 & 0 & 0.039 & -0.1318 & 0.041132 & -0.139005 & 0 & 0 \\
\hline 0) & 10 & 0.049 & 2.6235 & 0.0516787 & 2.766918 & 0 & 3 \\
\hline 0 & 15 & 0.0545 & 3.99 & 0.0574793 & 4.20812 & 0 & 4.5 \\
\hline 의 & 20 & 0.053 & 5.391 & 0.0558973 & 5.685708 & 0 & 6 \\
\hline 5 & 5 & 1.395 & 1.242 & 1.47126 & 1.309896 & 1.5 & 1.5 \\
\hline 5 & -5 & 1.386 & -1.542 & 1.461768 & -1.626296 & 1.5 & -1.5 \\
\hline-5 & 5 & $-1,315$ & 1.252 & -1.386887 & 1.3204427 & -1.5 & 1.5 \\
\hline-5 & -5 & -1.318 & -1.541 & -1.390051 & -1.625241 & -1.5 & -1.5 \\
\hline 10 & 10 & 2.788 & 2.647 & 2.9404107 & 2.7917027 & 3 & 3 \\
\hline-10 & 10 & -2.664 & 2.616 & -2.809632 & 2.759008 & -3 & 3 \\
\hline 10 & 0 & 2.764 & -0.01327 & 2.9150987 & -0.013995 & 3 & 0 \\
\hline-10 & 0 & -2.666 & -0.142 & -2.811741 & -0.149763 & -3 & 0 \\
\hline 10 & -10 & 2.773 & -2.903 & 2.9245907 & -3.061697 & 3 & -3 \\
\hline 15 & 15 & 4.235 & 4.093 & 4.4665133 & 4.3167507 & 4.5 & 4.5 \\
\hline-15 & 15 & -3.957 & 4.005 & -4.173316 & 4.22394 & -4.5 & 4.5 \\
\hline 15 & 이 & 4.2 & -0.129 & 4.4296 & -0.136052 & 4.5 & 0 \\
\hline-15 & 0 & -3.985 & -0.174 & -4.202847 & -0.183512 & -4.5 & 0 \\
\hline 15 & -15 & 4.175 & -4.25 & 4.4032333 & -4.482333 & 4.5 & -4.5 \\
\hline-15 & -15 & -3.983 & -4.308 & -4.200737 & -4.543504 & -4.5 & -4.5 \\
\hline 20 & 0 & 5.668 & -0.127 & 5.9778507 & -0.133943 & 6 & 0 \\
\hline-20 & 0) & -5.291 & -0.19 & -5.580241 & -0.200387 & -6 & 0 \\
\hline 0 & -20 & 0.0407 & -5.6641 & 0.0429249 & -5.973737 & 이 & -6 \\
\hline 0 & -15 & 0.0407 & -4.2894 & 0.0429249 & -4.523887 & 아 & -4.5 \\
\hline 0 & -10 & 0.0369 & -2.9202 & 0.0389172 & -3.079838 & 이 & -3 \\
\hline
\end{tabular}




\section{Interferometry data for Phase-plate optic}

Coordinates in table are for one element the other element would be moved just the opposite. Blanks indicate the Wyko was unable to get an accurate measurement for that position.

\begin{tabular}{|c|c|c|c|c|c|}
\hline 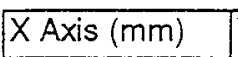 & $Y$ Axis $(\mathrm{mm})$ & $P-V(633 \mathrm{~nm})$ & $P-V(600 \mathrm{~nm})$ & ideal PV & Residual RMS \\
\hline 0 & -25 & 7.691 & 8.114005 & 7.5 & 0.041 \\
\hline 0 & -20 & 6.28 & 6.6254 & 6 & 0.038 \\
\hline 0 & -15 & 4.711 & 4.970105 & 4.5 & 0.032 \\
\hline 0 & -10 & 3.216 & 3.39288 & 3 & 0.029 \\
\hline 0 & -8 & 2.559 & 2.699745 & 2.4 & 0.026 \\
\hline 0 & $\overline{-6}$ & 1.948 & 2.05514 & 1.8 & 0.03 \\
\hline 0 & -4 & 1.369 & 1.444295 & 1.2 & 0.026 \\
\hline 0 & -2 & 0.76 & 0.8018 & 0.6 & 0.029 \\
\hline 0 & -1.5 & 0.586 & 0.61823 & 0.45 & 0.032 \\
\hline 0 & -1 & 0.457 & 0.482135 & 0.3 & 0.033 \\
\hline 0 & -0.5 & 0.365 & 0.385075 & 0.15 & 0.036 \\
\hline 0 & 0 & 0.148 & 0.15614 & 0 & 0.035 \\
\hline 0 & 0.5 & 0.243 & 0.256365 & 0.15 & 0.034 \\
\hline 0 & 1 & 0.373 & 0.393515 & 0.3 & 0.035 \\
\hline 0 & 1.5 & 0.512 & 0.54016 & 0.45 & 0.031 \\
\hline 0 & 2 & 0.7 & 0.7385 & 0.6 & 0.027 \\
\hline 0 & 4 & 1.295 & 1.366225 & 1.2 & 0.027 \\
\hline 0 & 6 & 1.86 & 1.9623 & 1.8 & 0.029 \\
\hline 0 & 8 & 2.555 & 2.695525 & 2.4 & 0.027 \\
\hline 0 & 10 & 3.154 & 3.32747 & 3 & 0.029 \\
\hline 0 & 15 & 4.658 & 4.91419 & 4.5 & 0.029 \\
\hline 0 & 20 & 6.18 & 6.5199 & 6 & 0.03 \\
\hline 0 & 25 & 8.148 & 8.59614 & 7.5 & \\
\hline 25 & o & 4.709 & 4.967995 & 7.5 & \\
\hline 20 & 0 & 4.949 & 5.221195 & 6 & \\
\hline 15 & 0 & 4.738 & 4.99859 & 4.5 & 0.034 \\
\hline 10 & 0 & 3.158 & 3.33169 & 3 & 0.028 \\
\hline 8 & 0 & 2.563 & 2.703965 & 2.4 & 0.031 \\
\hline 6 & 0 & 1.933 & 2.039315 & 1.8 & 0.029 \\
\hline 4 & 0 & 1.308 & 1.37994 & 1.2 & 0.025 \\
\hline 2 & 0 & 0.731 & 0.771205 & 0.6 & 0.025 \\
\hline 1.5 & o & 0.611 & 0.644605 & 0.45 & 0.02 \\
\hline 1 & 0 & 0.405 & 0.427275 & 0.3 & 0.022 \\
\hline 0.5 & 0 & 0.248 & 0.26164 & 0.15 & 0.031 \\
\hline 0 & 0 & 0.172 & 0.18146 & 0 & 0.035 \\
\hline-0.5 & 0 & 0.293 & 0.309115 & 0.15 & 0.033 \\
\hline-1 & 0 & 0.412 & 0.43466 & 0.3 & 0.027 \\
\hline-1.5 & 0) & 0.587 & 0.619285 & 0.45 & 0.02 \\
\hline-2 & 이 & 0.746 & 0.78703 & 0.6 & 0.023 \\
\hline-4 & 0 & 1.357 & 1.431635 & 1.2 & 0.024 \\
\hline
\end{tabular}




\begin{tabular}{|r|r|r|r|r|r|}
\hline-6 & 0 & 1.995 & 2.104725 & 1.8 & 0.03 \\
\hline-8 & 0 & 2.646 & 2.79153 & 2.4 & 0.03 \\
\hline-10 & 0 & 3.401 & 3.588055 & 3 & 0.028 \\
\hline-15 & 0 & 6.516 & 6.87438 & 4.5 & \\
\hline-20 & 0 & 5.794 & 6.11267 & 6 & \\
\hline-25 & 0 & 4.465 & 4.710575 & 7.5 & \\
\hline 1 & 1 & 0.621 & 0.655155 & 0.6 & 0.019 \\
\hline-1 & 1 & 0.629 & 0.663595 & 0.6 & 0.031 \\
\hline-1 & -1 & 0.67 & 0.70685 & 0.6 & 0.022 \\
\hline 1 & -1 & 0.677 & 0.714235 & 0.6 & 0.028 \\
\hline 5 & 5 & 2.963 & 3.125965 & 3 & 0.03 \\
\hline-5 & 5 & 3.03 & 3.19665 & 3 & 0.029 \\
\hline-5 & -5 & 3.082 & 3.25151 & 3 & 0.029 \\
\hline 5 & -5 & 3.047 & 3.214585 & 3 & 0.029 \\
\hline 10 & 10 & 5.934 & 6.26037 & 6 & 0.031 \\
\hline-10 & 10 & 6.01 & 6.34055 & 6 & 0.029 \\
\hline-10 & -10 & 9.336 & 9.84948 & 6 & \\
\hline 10 & -10 & 5.955 & 6.282525 & 6 & 0.032 \\
\hline 15 & 15 & 9.458 & 9.97819 & 9 & \\
\hline & & & & & \\
\hline
\end{tabular}




\section{Sag data for Phase-plate optic}

\begin{tabular}{|r|r|r|r|r|r|r|r|}
\hline$x$ & $y$ & sag_x & \multicolumn{1}{l}{ sag_y } & \multicolumn{1}{l}{$\begin{array}{l}\text { sag_x } \\
@ 600 n m\end{array}$} & $\begin{array}{l}\text { sag_y } \\
@ 600 n m\end{array}$ & $\begin{array}{l}\text { ideal } \\
\text { sag_x }\end{array}$ & ideal sag_y \\
\hline & 0 & $4.93 E-04$ & 0.0509 & 0.000520115 & 0.0536995 & 0 & 0 \\
\hline 20 & 0 & -5.4428 & -0.0662 & -5.742154 & -0.069841 & -6 & 0 \\
\hline 15 & 15 & -4.1132 & -4.4212 & -4.339426 & -4.664366 & -4.5 & -4.5 \\
\hline 15 & -15 & -3.766 & 4.1569 & -3.97313 & 4.3855295 & -4.5 & 4.5 \\
\hline 15 & 0 & -4.1155 & -0.0954 & -4.3418525 & -0.100647 & -4.5 & 0 \\
\hline 10 & 10 & -2.7344 & -2.9332 & -2.884792 & -3.094526 & -3 & -3 \\
\hline 10 & -10 & -2.8118 & 2.8466 & -2.966449 & 3.003163 & -3 & 3 \\
\hline 10 & 0 & -2.7806 & -0.0649 & -2.933533 & -0.0684695 & -3 & 0 \\
\hline 5 & 5 & -1.374 & -1.4441 & -1.44957 & -1.5235255 & -1.5 & -1.5 \\
\hline 5 & -5 & -1.4094 & 1.4541 & -1.486917 & 1.5340755 & -1.5 & 1.5 \\
\hline 0 & 20 & 0.0793 & -5.7181 & 0.0836615 & -6.0325955 & 0 & -6 \\
\hline 0 & 15 & 0.0661 & -4.2768 & 0.0697355 & -4.512024 & 0 & -4.5 \\
\hline 0 & 10 & 0.0432 & -2.8513 & 0.045576 & -3.0081215 & 0 & -3 \\
\hline 0 & -20 & -0.071 & 5.8736 & -0.074905 & 6.196648 & 0 & 6 \\
\hline 0 & -15 & -0.0505 & 4.3868 & -0.0532775 & 4.628074 & 0 & 4.5 \\
\hline 0 & -10 & -0.0322 & 2.9472 & -0.033971 & 3.109296 & 0 & 3 \\
\hline-20 & 0 & 6.1058 & 0.2477 & 6.441619 & 0.2613235 & 6 & 0 \\
\hline-15 & 15 & 3.664 & -3.9215 & 3.86552 & -4.1371825 & 4.5 & -4.5 \\
\hline-15 & -15 & 4.8758 & 4.6085 & 5.143969 & 4.8619675 & 4.5 & 4.5 \\
\hline-15 & 0 & 4.7447 & 0.0826 & 5.0056585 & 0.087143 & 4.5 & 0 \\
\hline-10 & 10 & 2.8855 & -2.7668 & 3.0442025 & -2.918974 & 3 & -3 \\
\hline-5 & 5 & 1.4303 & -1.3484 & 1.5089665 & -1.422562 & 1.5 & -1.5 \\
\hline-5 & -5 & 1.3956 & 1.5099 & 1.472358 & 1.5929445 & 1.5 & 1.5 \\
\hline-10 & -10 & 2.8686 & 3.0746 & 3.026373 & 3.243703 & 3 & 3 \\
\hline-10 & 0 & 2.8459 & 0.1326 & 3.0024245 & 0.139893 & 3 & 0 \\
\hline
\end{tabular}




\section{Chapter 3 - Heterodyne Wavefront Sensor Report}

\section{Rob Hartley}

1. General Description. Current wavefront control systems use a Hartmann sensor as the wavefront sensor. The Hartmann sensor consists of a lenslet array in front of a CCD camera. The lenslets spatially sample the wavefront, with each lenslet forming a spot on the CCD. Changes in the wavefront slope impinging on the lenslet are transformed into spot centroid motion on the CCD. The wavefront phase errors corresponding to the wavefront slope measurements must be reconstructed in the computer processing the camera data. To accomplish this, all of the Hartmann spot centroids must be calculated, and a vector matrix multiply applied to each frame of the camera data.

A heterodyne sensor figure 1. would use a lenslet array coupled to an array of photodiodes. The process mean to be corrected is mixed with a frequency shifted reference beam derived from the master oscillator in the process beam train, and falls on the photodiode array. The difference frequency of the two beams appears at the - photodiode output, with the phase of the electronic signal directly proportional to the phase of the process beam with respect to the reference beam. The phase error signals can be directly applied to the wavefront corrector. The motivation of developing this wavefront control approach is that the costly control computer of the traditional approach can potentially be eliminated. A second feature of this wavefront sensor technique is that it is much higher speed than camera based sensors. This may be advantageous for AVLIS, NIF, and DOD applications. figure 2. 


\section{Conventional (Hartmann) WFS measures local wavefront tilt, heterodyne WFS measures phase}

\section{Hartmann sensor}

Incident

Wavefront

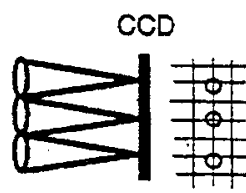

Heterodyne sensor

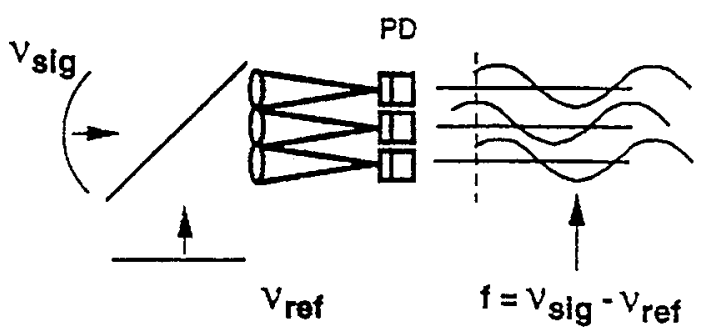

- Output is sine wave electrical signal, of trequency $f$

- Relative phase between sampled wavefront positions measured directly

- Large dynamic range, phase information independent of intensity

- Wavefront reference can be derlved from process beam

- Wavefront reference source (not shown), also needed to callbrate

figure 1. Hartmann Sensor vs. Heterodyne Sensor 


\section{Heterodyne WFC approach has potentlal for significant complexity and cost reduction}

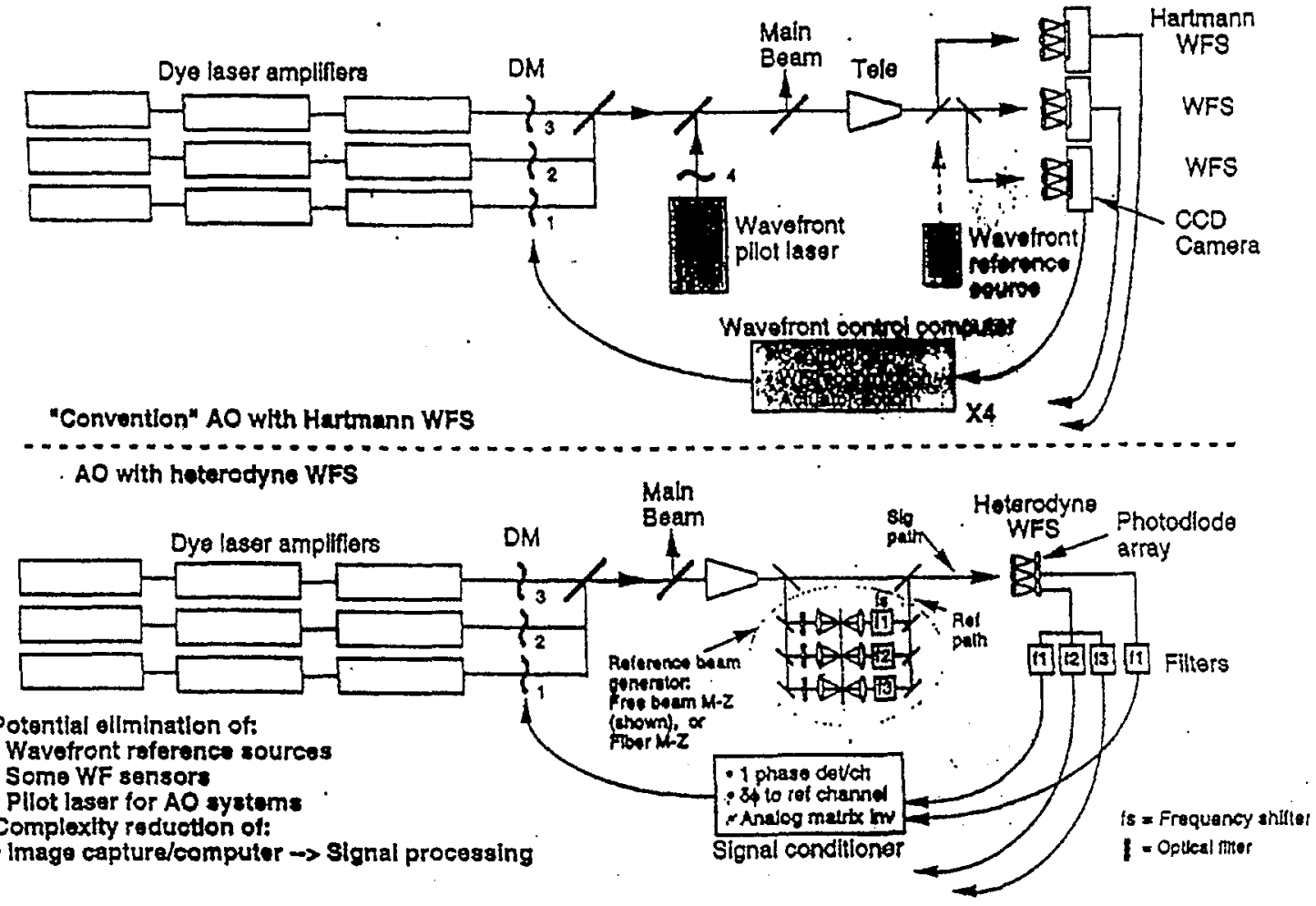

(A) 071897-4

figure 2.

Current B490 Hartmann sensor application (top) and proposed 'plant' heterodyne sensor application (bottom). 
2. Approach. The vision for a heterodyne adaptive optic sensor was to start with a simple one or two channel photodetector system and build a closed loop control system around this sensor to demonstrate proof of concept. The next step foreseen was to increase beam size and the number of channels in our control loop.

Heterodyne sensor development has since sequenced through four areas. First, basic RF work was initiated to confirm the heterodyne idea. Secondly, this RF work graduated to the use of a single line HeNe and simple optical circuit of splitters and combiners for further heterodyne and phase detection development. Next, investigation into heterodyning and phase detection using B490 Dye Master Oscillator (DMO) light was conducted to confirm applicability to plant. Lastly design an array of photo detectors for a multi-channel application. A summary of accomplishments and results follows.

-3. Accomplishments and results. Using and RF signal generator. figure 3. a 40Mhz phase shift circuit using phase detector built from a pair of mixers was built. Eventually a commercial IQ modulator / phase detector was used. By using a RF trombone, the phase between two $40 \mathrm{Mhz}$ signals was observed figure 4 .

Het Sensor RF Lab Set Up 3/3/98

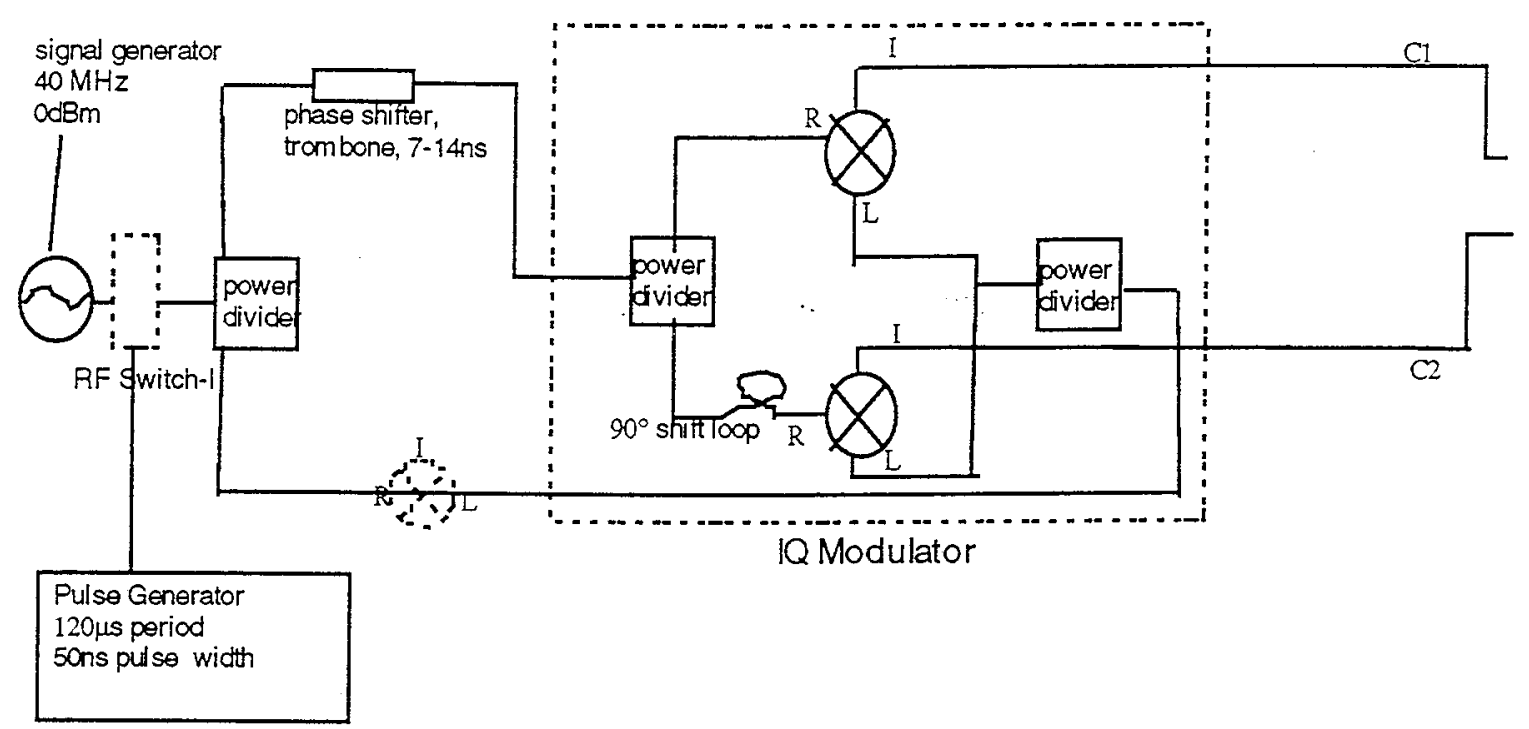

figure 3. 

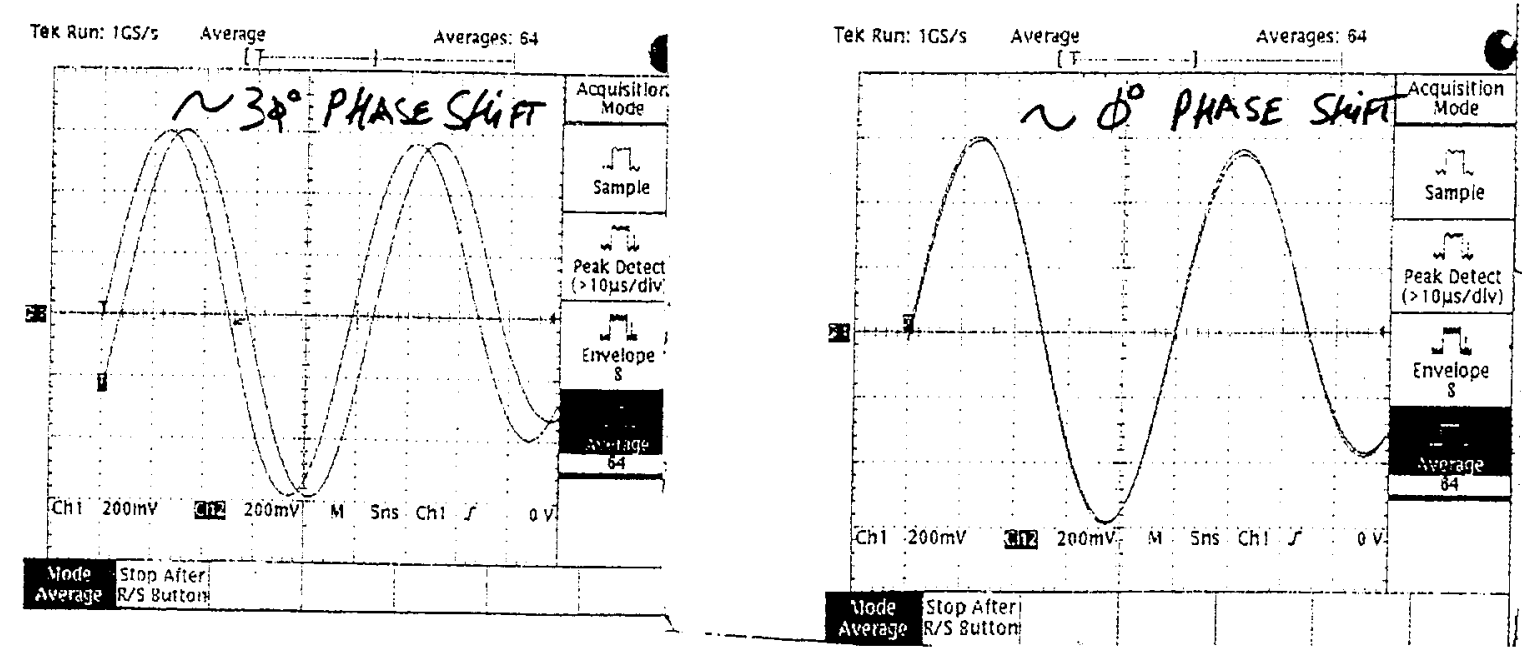

figure 4.

Adding phase between two signals. By moving the phase shifter, a trombone waveguide, phase shift can be created.

The above signals are fed into a phase shifter / IQ modulator. Below in figure 5, one voltage is at an apparent minimum, while the sin has reaching a maximum.

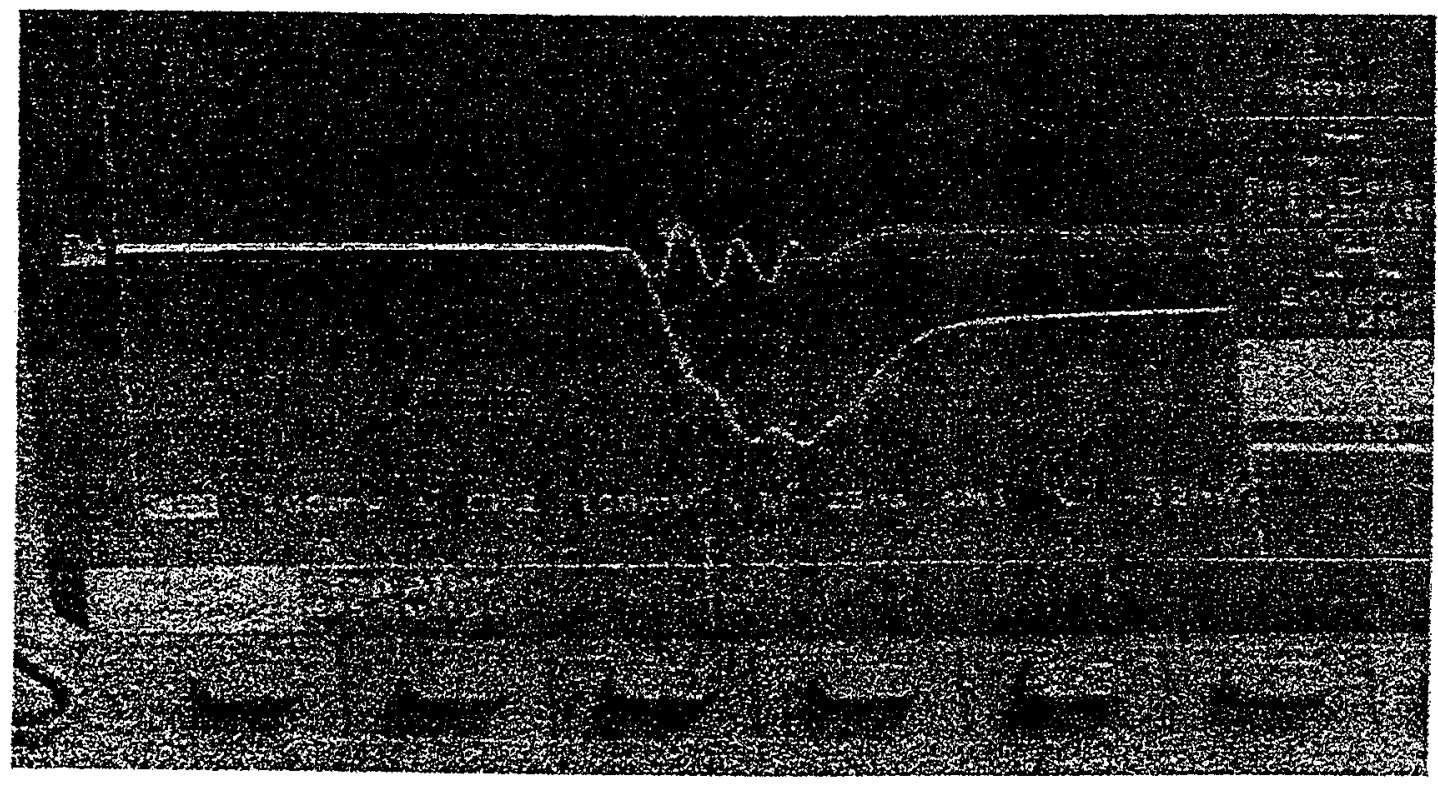

figure 5 .

I and Q DC voltages change linearly to the amount of phase seen in figure 4. 
I and Q DC voltages from the phase detector / IQ modulator were plotted, figure 6., using the relationship:

$$
\tan ^{-1}(I / Q)=\theta .
$$

\section{Dynamic Range}

\section{IQ Modulator}

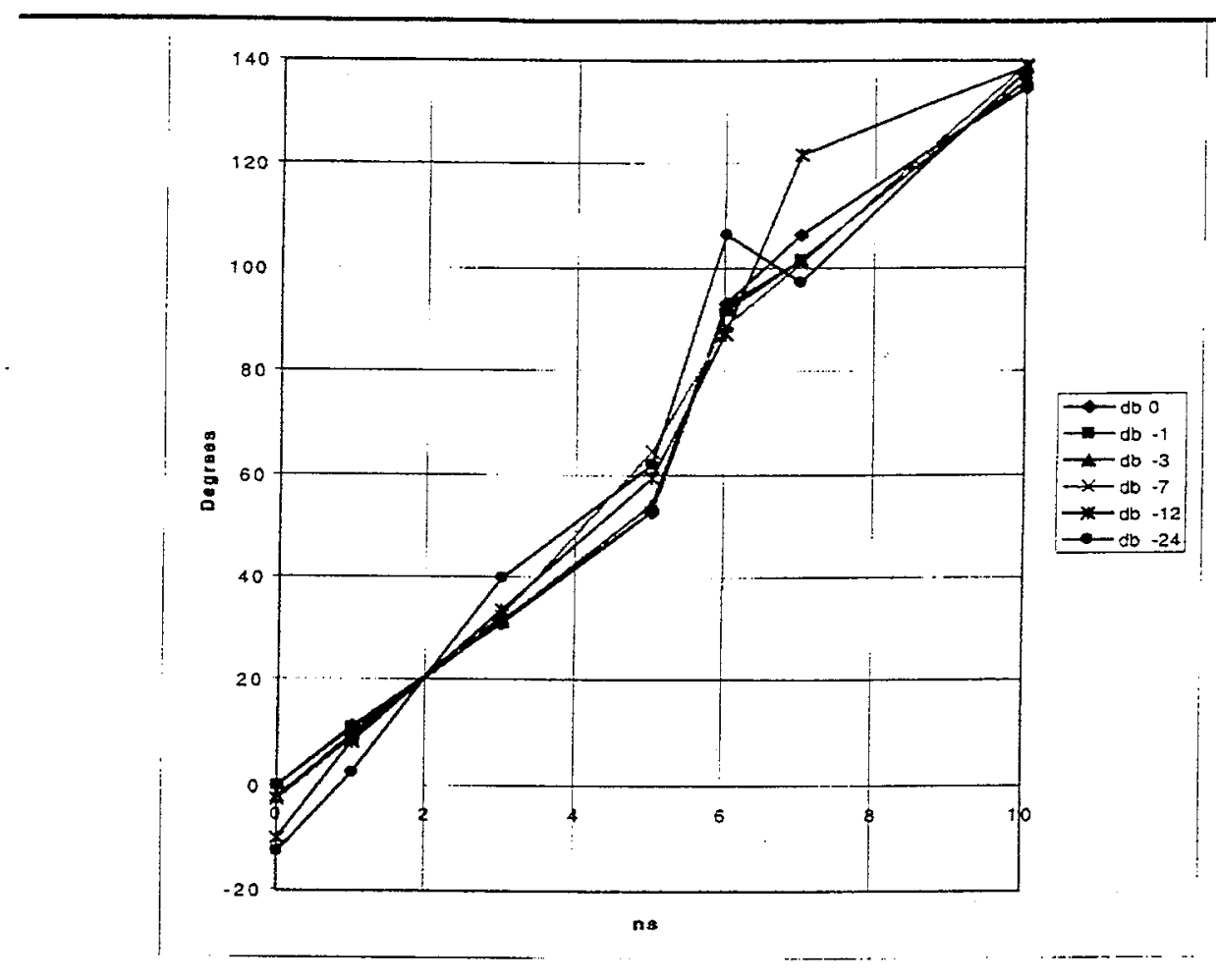

figure 6.

A fairly linear plot of Ns phase delays fed into the phase detector versus calculated phase delays using IQ modulator output voltages.

$40 \mathrm{Mhz}$ work ended and an optical heterodyne circuit was built using a single line HeNe. figure 4 . The circuit was set up to include (CW) laser source, a 200Mhz acousto optic modulator (AOM) / phase shifter, beam splitters, combiners and two photodetectors with preamplifiers. These photodetectors were placed at a location to observe the two 
heterodyned optical paths, one of which was shifted by 200Mhz. A 13 actuator deformable mirror (DFM) was installed into one of the optical paths.

A closed loop control was built to lock the two optical paths together in the CW mode using one actuator of the 13 actuator DFM. Later the circuit was modified to operate in the 'plant typical' pulsed mode by using a second acousto optic modulator as a chopper. 200Mhz signals produced by heterodyning were observed in the pulsed mode and $\mathrm{CW}$ mode.

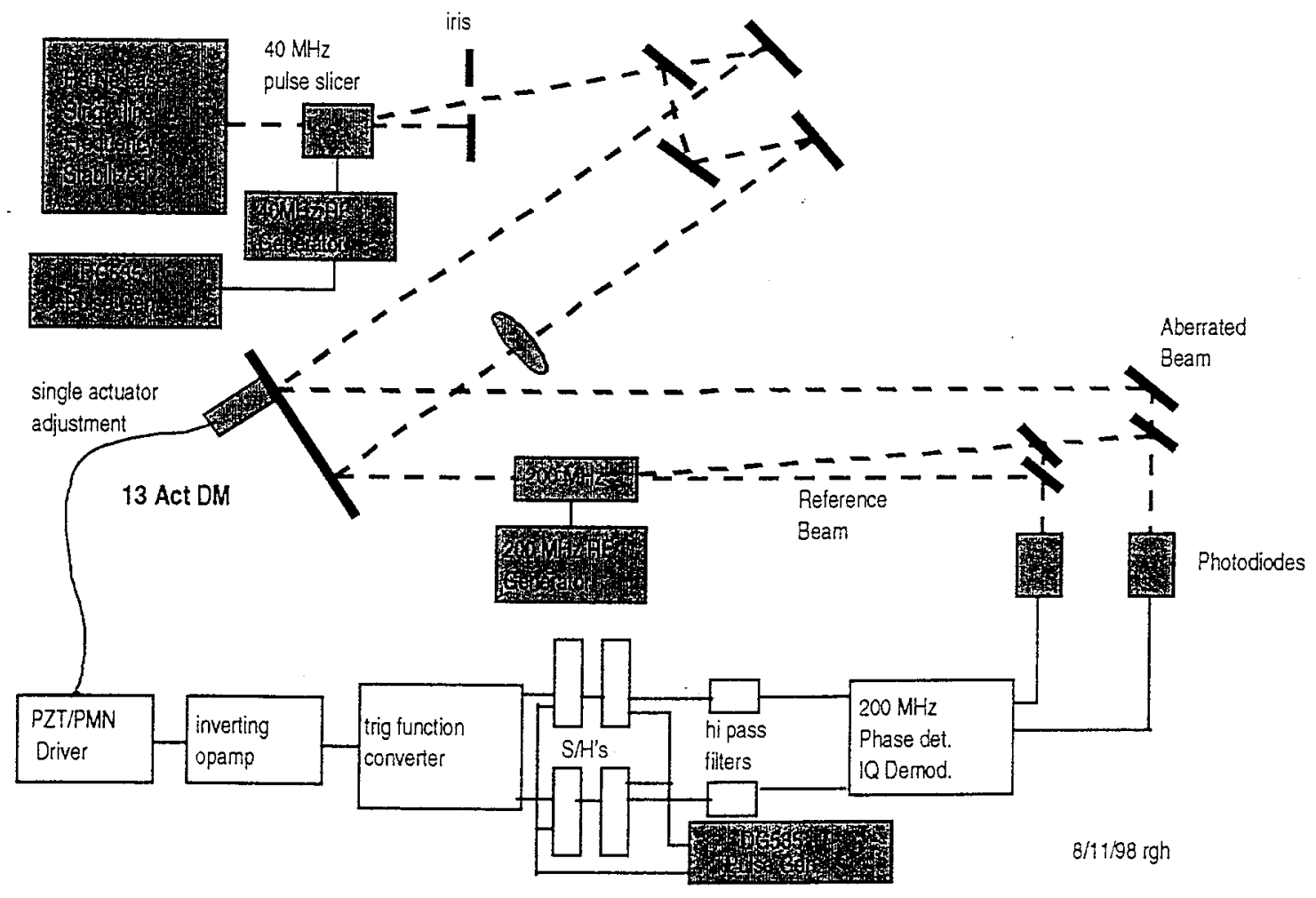

figure 4. Single Line HeNe Heterodyne Experiment

The following 350ns envelope was observed with our HeNe heterodyne experiment indicating a $200 \mathrm{Mhz}$ modulation on top of the light pulse. figure 5 . 


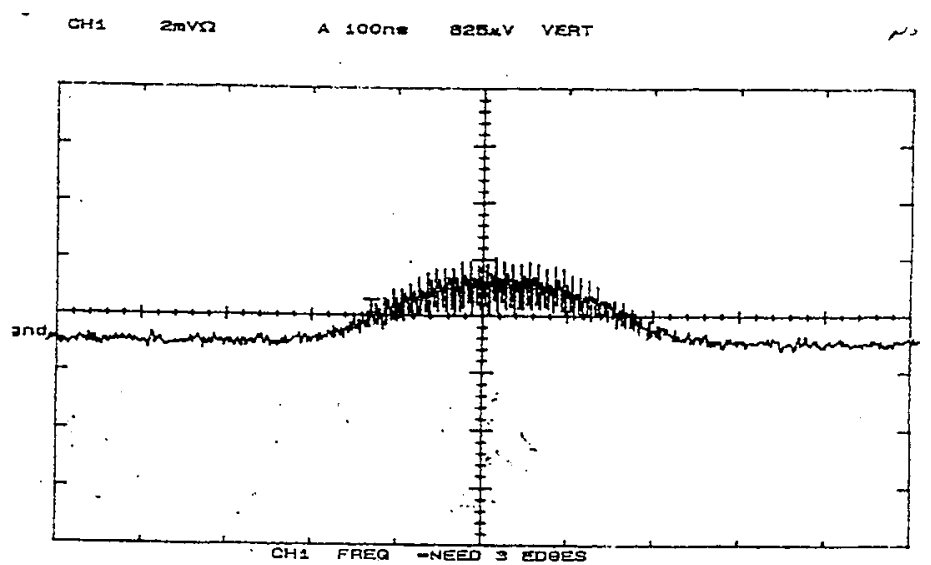

figure 5.

After passing the signal above through a high pass filter, the $200 \mathrm{Mhz}$ modulation centered about ground. figure 6.

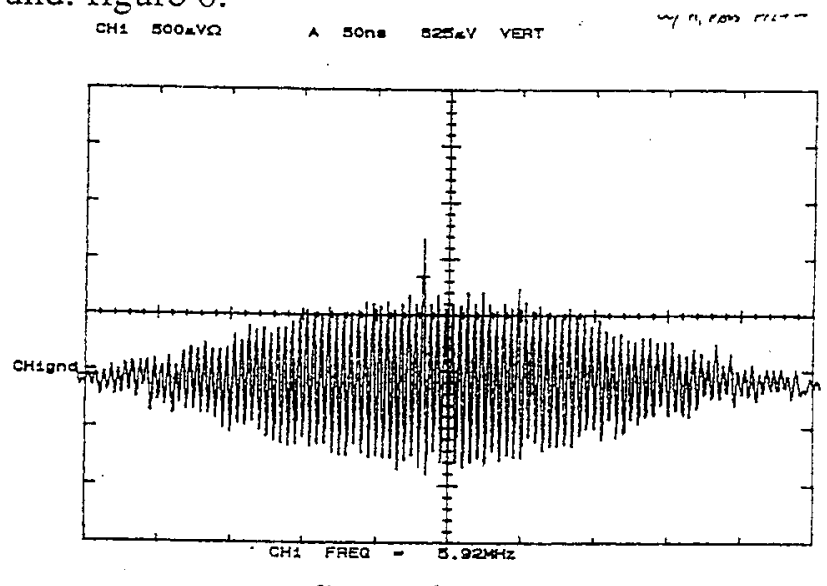

figure 6.

Using a $100 \mathrm{Mhz}$ acousto optic modulator similar results were observed. figure 7 . The $100 \mathrm{Mhz}$ AOM was set up to investigate whether heterodyning could be achieved to use cheaper, lower bandwidth photodetectors. A sacrifice observed is that with a given pulse width, $100 \mathrm{Mhz}$ has fewer periods for the IQ modulator to observe. 

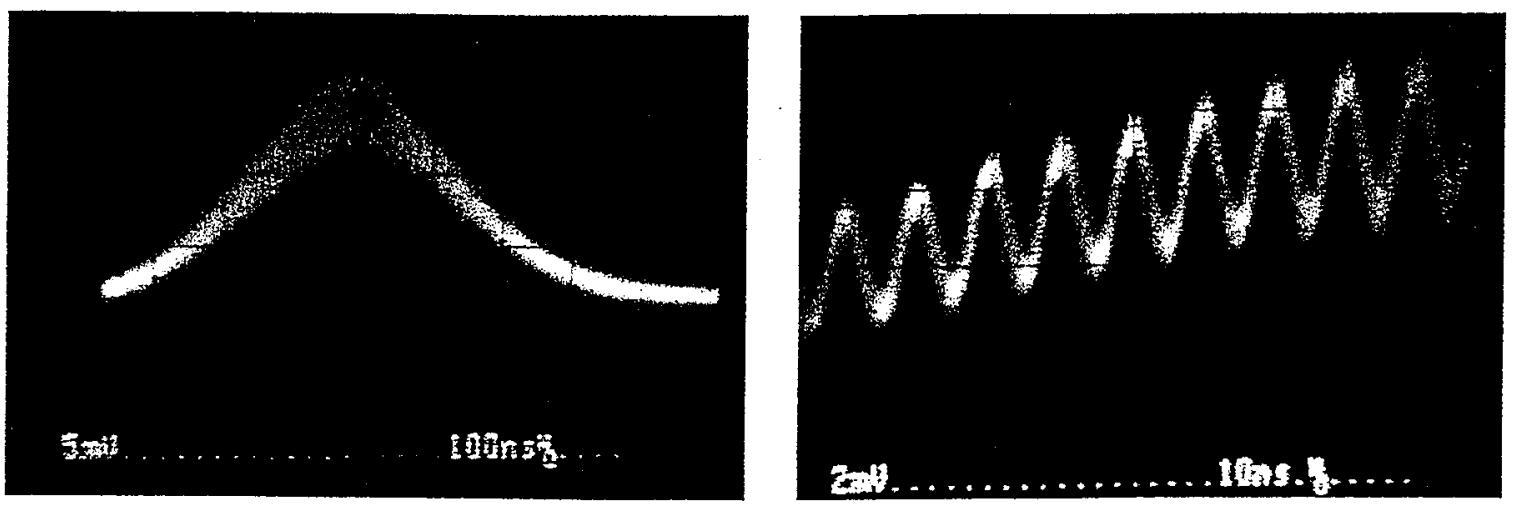

figure 7.

heterodyne pulse with modulation revealing $10 \mathrm{~ns}$ period

We confirmed our heterodyned derived $200 \mathrm{Mhz}$ signal with a spectrum analyzer plot shown. figure 8.

worse Cositis

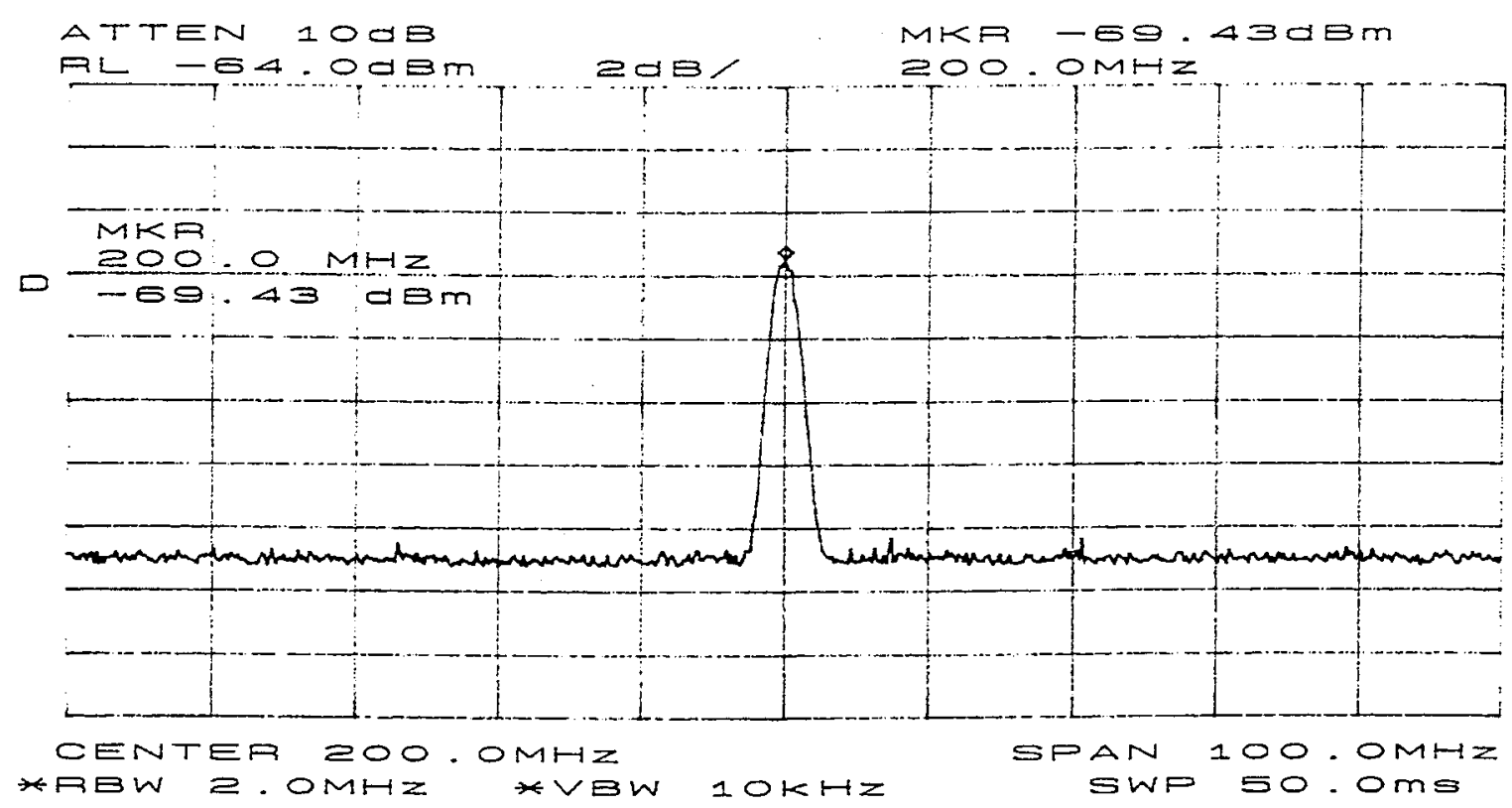

figure 8 .

A closed loop controller was built. The following scope traces taken. When the controller held the two optical paths locked, better than $1 / 10$ wave resolution was observed. figure 9. 
CW unlocked photodiodes'

output

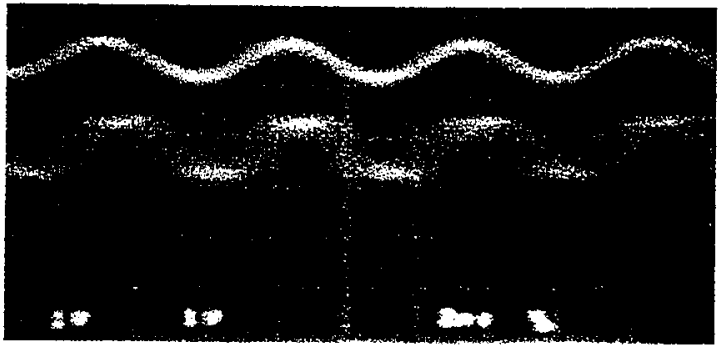

controller in open loop. two $\mathrm{CW}$ beams not locked.

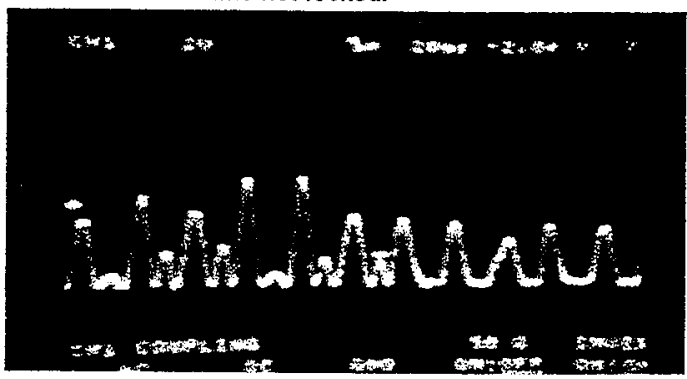

CW locked photodiodes'

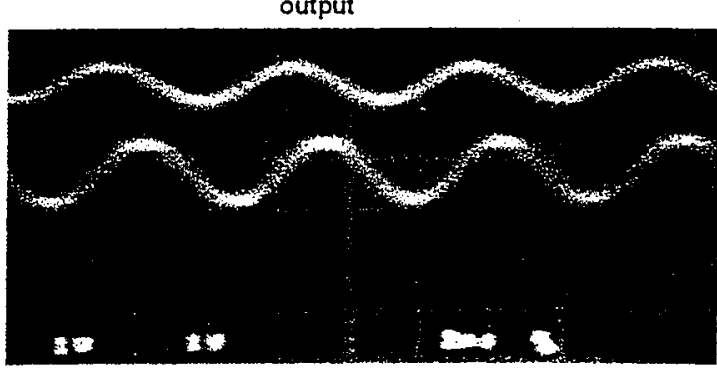

controller in closed loop locking two $\mathrm{CW}$ beams of light together

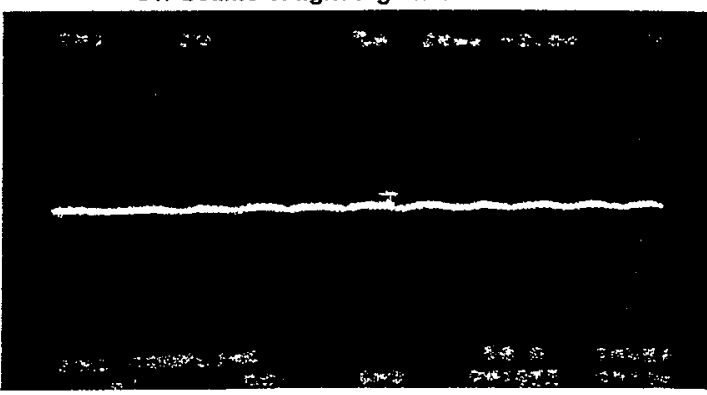

figure 9.

The experiment was repeated using pulsed HeNe light. We observed the following results. figure 10 . 


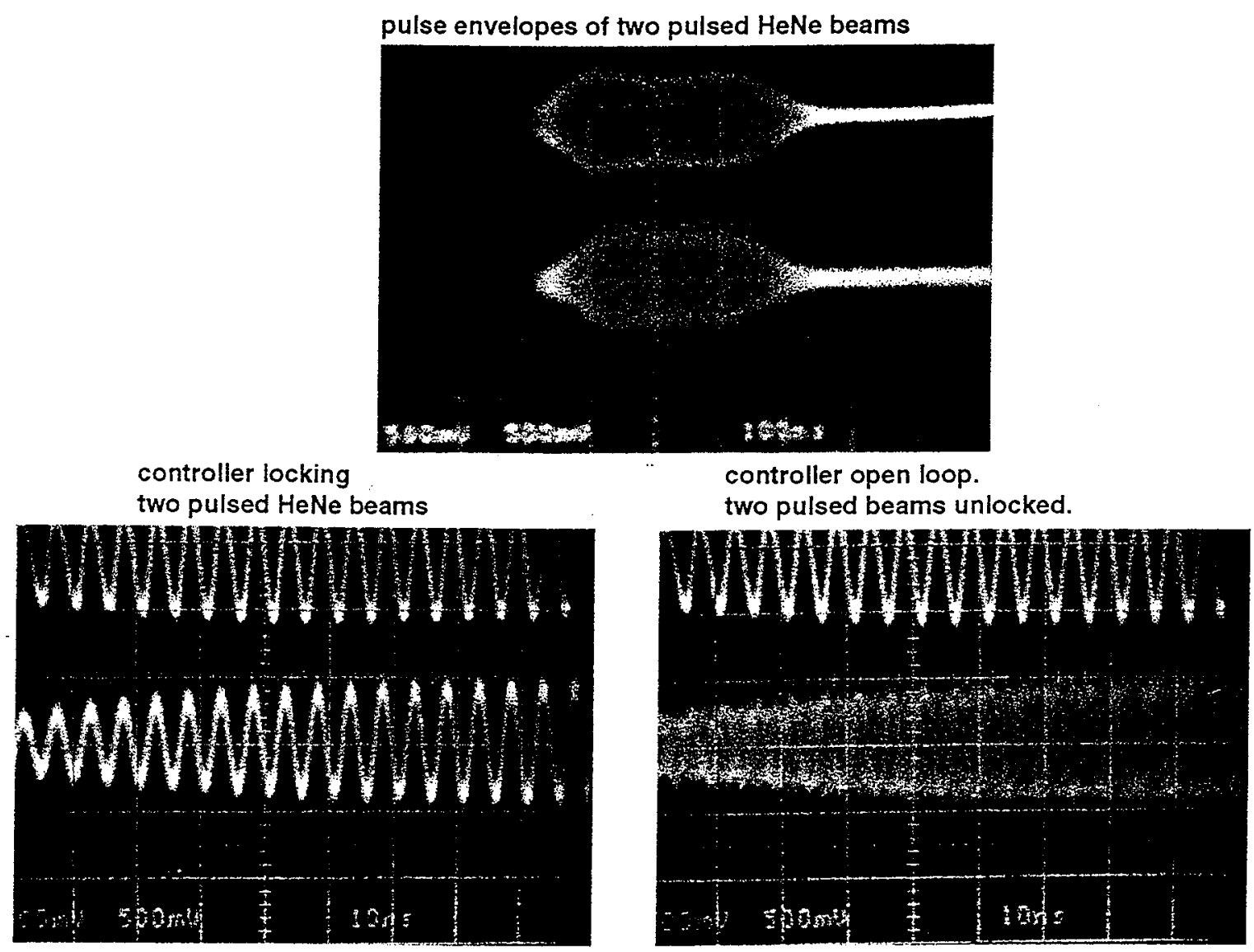

figure 10.

The experiment was now set up using Dye Master Oscillator (DMO). Using fibredelivered, modulated and unmodulated B490 Dye Master Oscillator Light (DMO). figure 11. Using pulsed DMO light, $200 \mathrm{Mhz}$ signals with and without modulation were observed. The circuit below, figure 10 , allowed us to determine whether a bandpass filter was needed. Paths matched to $.01 \mathrm{~ns} / .1$ " to the combiner plate negated the need for a bandpass filter however several commercial combinations of low pass and high pass filters were initially tested. Figure 12. demonstrates that the better match paths are to the combiner plate, the less frequencies are observed on the spectrum plot. 
het sensor DMO drawing

26 Jan 99

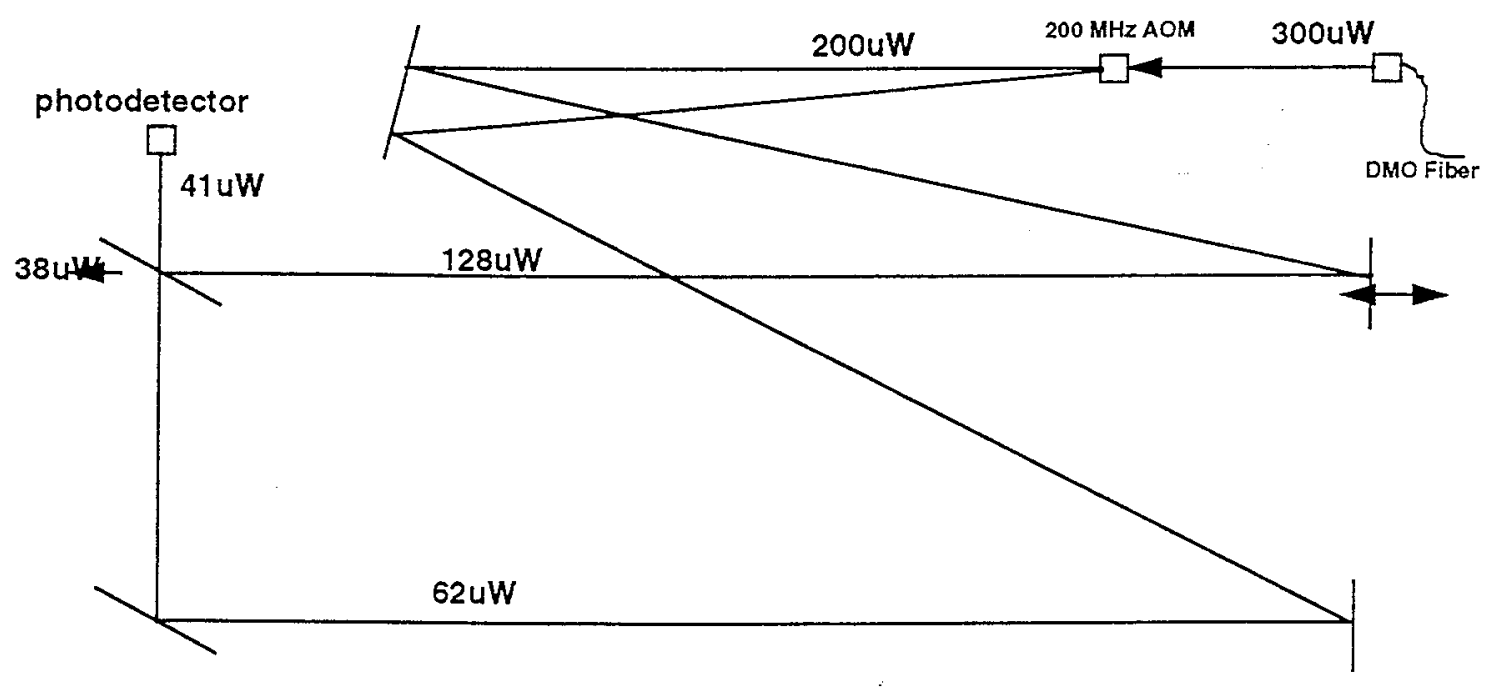

figure 11. DMO experiment layout 

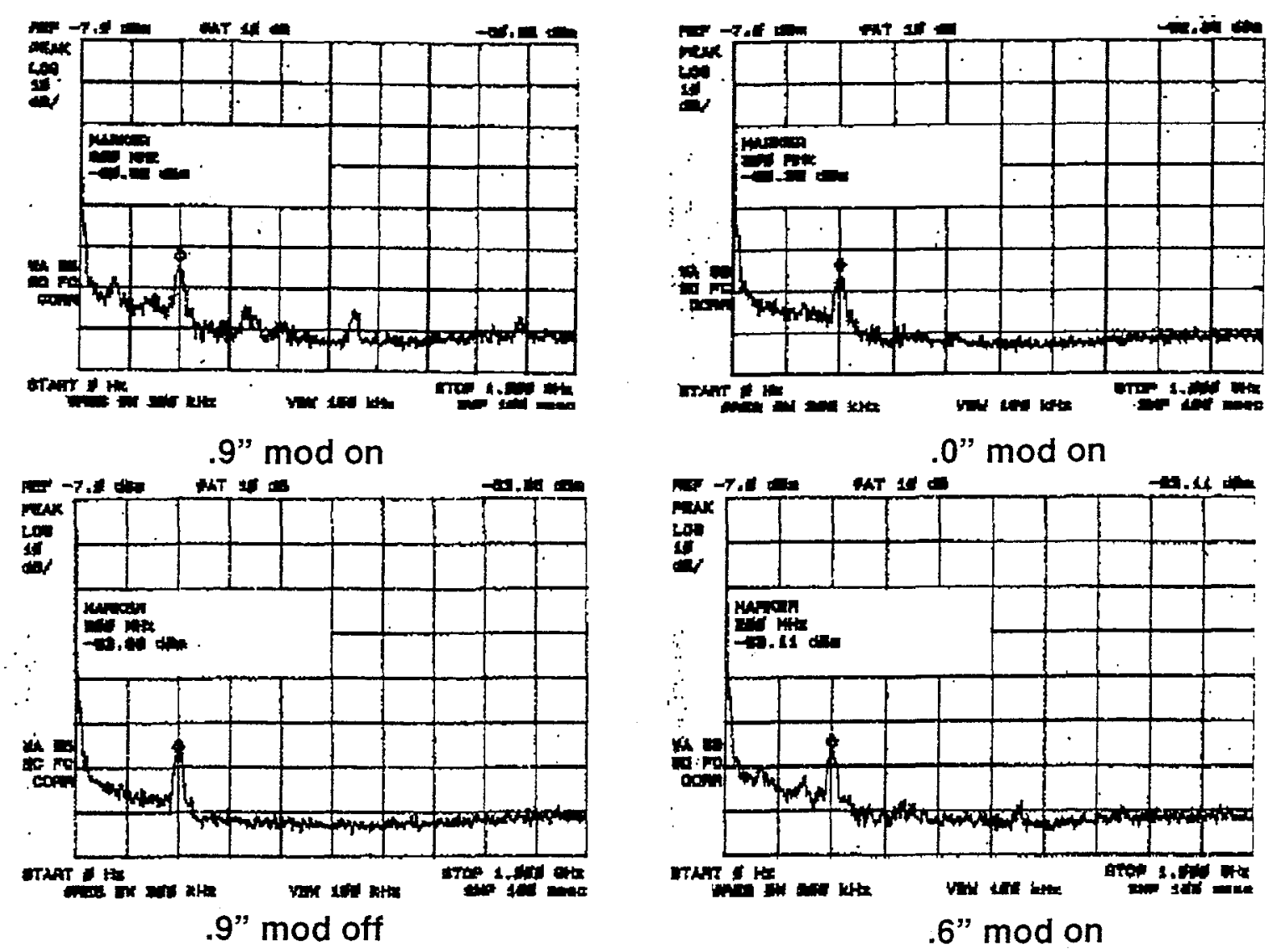

figure 12.

Effects of mismatched path differences

We are planning to conduct a full beam het sensor experiment in the AW4 diagnostics. figure 13 . 


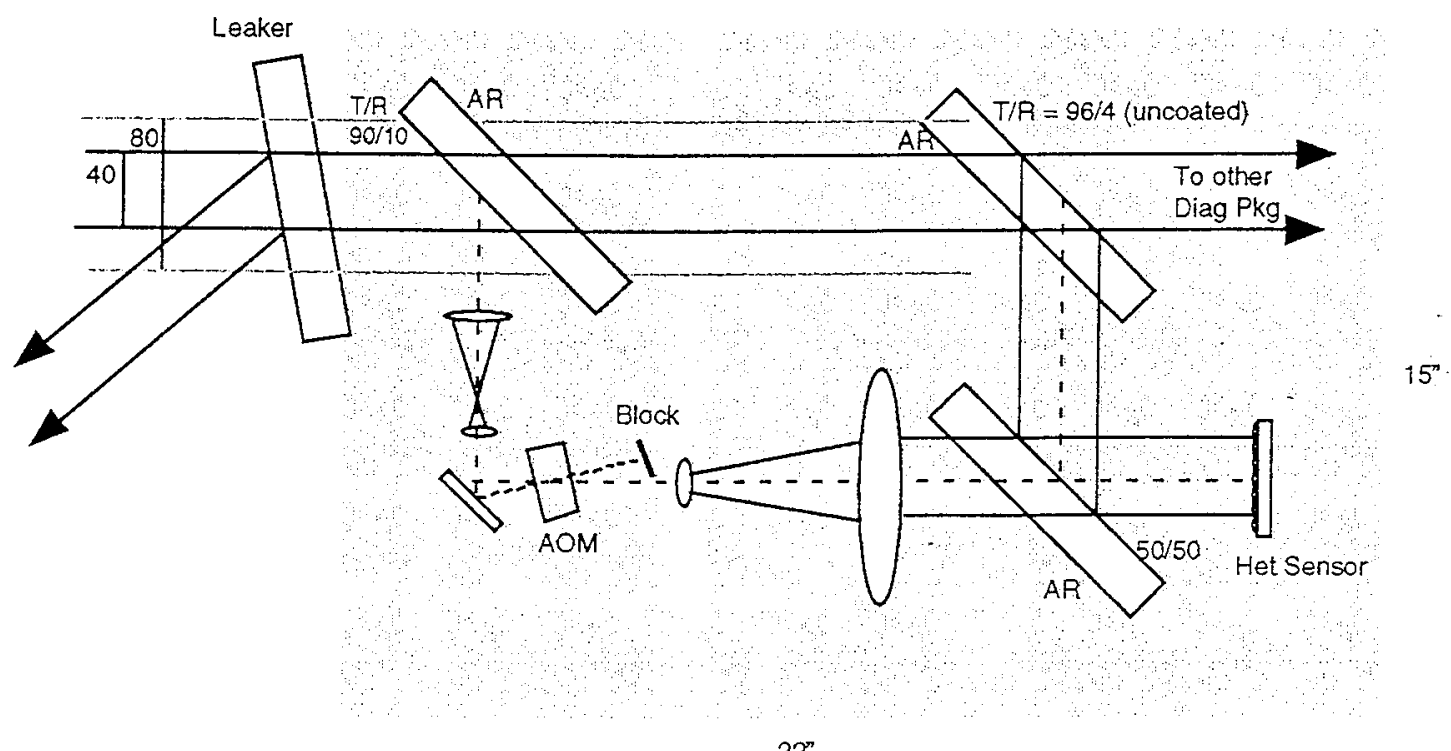

$22^{n}$

figure 13.

Full beam het sensor experiment

Desiring a high modulation index, it was determined that the power in both beams, the shifted reference and the non shifted beam should be as equal as possible. The spreadsheet below, fig 14. was used as a model to predict how well we could match power of the the two beams, while retaining suitable intensity on our photodetectors and depth of modulation .

\section{L1-L2-L3-lens-photodetector leg}

\begin{tabular}{|c|c|c|c|}
\hline 1 & into $L 1$ we put & 3000 & $\mathrm{~mW}$ \\
\hline 2 & if $L 1$ leaks to us & $50 \%$ & \\
\hline 3 & into $L 2$ we see & 1500 & $\mathrm{~mW}$ \\
\hline 4 & into AW4 diag we see & 1500 & $\mathrm{~mW}$ \\
\hline 5 & if $L 2$ leaks to $L 3$ & $10 \%$ & \\
\hline 6 & into L3 we see & 150 & $\mathrm{~mW}$ \\
\hline 7 & if $L 3$ is a beam splitter of & $50 \%$ & \\
\hline 8 & into $3200 \mathrm{~mm}^{\wedge} 2$ array we see & 75 & $\mathrm{~mW}$ \\
\hline 9 & $\begin{array}{l}\text { with } 100: 1 \text { reduction onto } 32 \mathrm{~mm}^{\wedge} 2 \text { lens, the lens } \\
\text { sees }\end{array}$ & 0.75 & $\mathrm{~mW}$ \\
\hline 10 & the active region of photodetector then sees & 0.75 & $\mathrm{~mW}$ \\
\hline 11 & at $.5 \mathrm{AW}$, current out of photodetector is & 0.375 & A \\
\hline 12 & and into $50 \Omega$ we see & 18.75 & $\underline{m V}$ \\
\hline
\end{tabular}




\begin{tabular}{|c|c|c|c|c|c|}
\hline \multirow{8}{*}{$\begin{array}{l}\text { from \#5 } \\
\text { above }\end{array}$} & 13 & $\begin{array}{l}\text { L1-L2-SpatialFil-AOM-L3-lens-photodet leg } \\
\text { if } L 2 \text { leaks to } L 3\end{array}$ & $10 \%$ & & \\
\hline & 14 & $\begin{array}{l}\text { from } L 2 \text { into spatial filter we get } \\
\text { or }\end{array}$ & $\begin{array}{l}90 \% \\
1350\end{array}$ & $\mathrm{~mW}$ & across $3200 \mathrm{~mm}^{\wedge} 2$ \\
\hline & 15 & aperature only & 6.3 & $\mathrm{~mm} \varphi$ & \\
\hline & 16 & area aperatured is & 31.15665 & $\mathrm{~mm}^{\wedge} \mathrm{2}$ & \\
\hline & 17 & now we only get & 13.14421172 & $\mathrm{~mW}$ & into a cOTs telescope \\
\hline & 18 & if telescope is a reduce by & 7 & $x$ & \\
\hline & 19 & $\begin{array}{l}\text { collimated telescope output beam size is } \\
\text { which retains } \\
\text { (area is) }\end{array}$ & $\begin{array}{c}0.9 \\
0.63585 \\
13.14421172\end{array}$ & $\begin{array}{l}\mathrm{mm \varphi} \\
\mathrm{mm} \mathrm{m}^{\wedge} 2 \\
\mathrm{~mW}\end{array}$ & \\
\hline & 21 & if we spatial filter with a pinhole of & 20 & $\mu m$ & \\
\hline
\end{tabular}

figure 14. 


\title{
Chapter 4 - High-resolution wavefront control using liquid crystal spatial light modulators
}

\author{
Scot S. Olivier*, Michael W. Kartz, Brian J. Bauman, \\ James M. Brase, Curtis G. Brown, Jeffrey B. Cooke, \\ Deanna M. Pennington, Dennis A. Silva \\ Lawrence Livermore National Laboratory, PO Box 808, Livermore, CA 94551
}

\begin{abstract}
Liquid crystal spatial light modulator technology appropriate for high-resolution wavefront control has recently become commercially available. Some of these devices have several hundred thousand controllable degrees of freedom, more than two orders of magnitude greater than the largest conventional deformable mirror. We will present results of experiments to characterize the optical properties of these devices and to utilize them to correct aberrations in an optical system. We will also present application scenarios for these devices in high-power laser systems.
\end{abstract}

Keywords: adaptive optics, lasers, spatial light modulators, liquid crystals

\section{INTRODUCTION}

Precise wavefront control is critical for many high-power laser applications including inertial confinement fusion, laser isotope separation, and laser machining. There are many ways that laser wavefront quality can be degraded. Thermal effects due to the interaction of high-power laser or pump light with the internal optical components or with the ambient gas are common causes of wavefront degradation. For many years, adaptive optics based on thin deformable glass mirrors with piezoelectric or electrostrictive actuators have been used to remove the low-order wavefront errors from high-power laser systems. These adaptive optics systems have successfully improved laser beam quality, but have also often revealed additional high-spatial-frequency errors, both because the low-order errors have been reduced and because deformable mirrors have often introduced some high-spatial-frequency aberrations due to manufacturing errors. Many current laser applications could benefit from correction of high spatial frequency aberrations that can only be provided with correctors with thousands of degrees of freedom. This level of correction is beyond the state of the art of deformable mirror technology.

The largest deformable mirrors currently available have $~ 1000$ degrees of freedom at a cost of $\sim \$ 1 \mathrm{M}$. Even if the deformable mirror technology could be extended to more actuators, the cost would likely be prohibitive. Therefore, a new approach using a different wavefront control technology may be required. One new wavefront control approach is the use of liquid crystal (LC) spatial light modulator (SLM) technology for the control of the phase of linearly polarized light. Current LC SLM technology provides high-spatial-resolution wavefront control, with hundreds of thousands of degrees of freedom, more than two orders of magnitude greater than the largest deformable mirrors. Furthermore, even with the increased spatial resolution, the cost of these devices is nearly two orders of magnitude less than the cost of the largest deformable mirror.

\section{SLM TECHNOLOGY}

The LC SLM devices utilized in the investigations described in this paper are a variety of optically addressed (OA) nematic LC SLM currently available from both Jenoptik and Hammamatsu. These devices are capable of phase correction of greater than one wave at visible and near-infra-red wavelengths. An OA LC SLM consists of a thin-film sandwich structure, shown in Figure 1, with an amorphous silicon ( $\alpha$-Si) photo-semiconductor, a parallel aligned nematic liquid crystal, a dielectric mirror, and a pair of transparent electrodes on glass substrates. The voltage applied to the electrodes is divided between

\footnotetext{
* Correspondence: email: olivier1 @llnl.gov; phone: 925-423-6483; fax: 925-422-1796
} 
photo-semiconductor and liquid crystal layers, depending on the illumination intensity, thereby enabling modulation of the refractive index of the $L C$ by a $2 \mathrm{D}$ image.

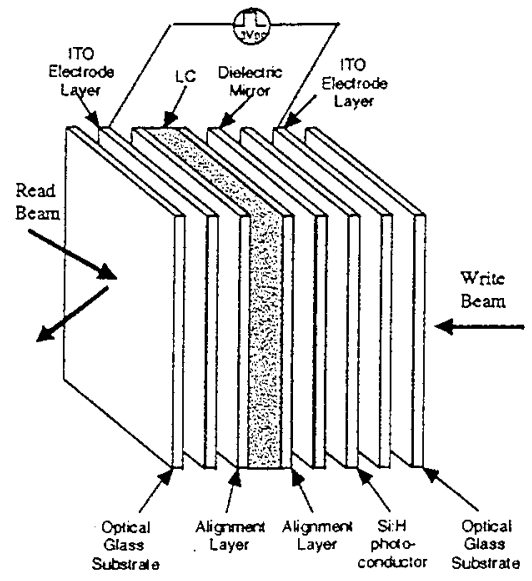

Figure 1. Schematic of optically addressed liquid crystal spatial light modulator

To activate the OA LC SLM the $\alpha$-Si layer must be exposed to an image pattern. The resolution of the SLM is directly proportional to the resolution of the image pattern projected onto the $\alpha$-Si layer up to some limit. In the state where no image is incident on the device, the impedance of the $\alpha$-Si layer is very high, with or without the voltage applied across the electrodes. When an image pattern is projected onto the $\alpha$-Si layer, the impedance of the $\alpha$-Si is reduced in proportion to the light intensity in that region, and the voltage applied to the liquid crystal is increased. This increased voltage causes an increase in the index of refraction in the liquid crystal layer due to reorientation of the liquid crystal molecules.

The resolution of the image projection system (which in this case is based on a liquid crystal display or LCD) determines at the resolution at which the SLM will effect the read beam. The currently available devices have the capability of providing control of up to 50 line-pairs/mm. The device from Jenoptik comes with a $832 \times 624$ LCD and Hamamatsu provides a $640 \times$ 480 LCD. The operational configuration is shown in Figure 2.

These devices work with polarized light. The maximum phase shift is obtained when the polarization of readout light is parallel to the axis of the liquid crystal molecules. When the polarization of readout light is perpendicular to the axis of the liquid crystal molecules, the phase shift is negligible.

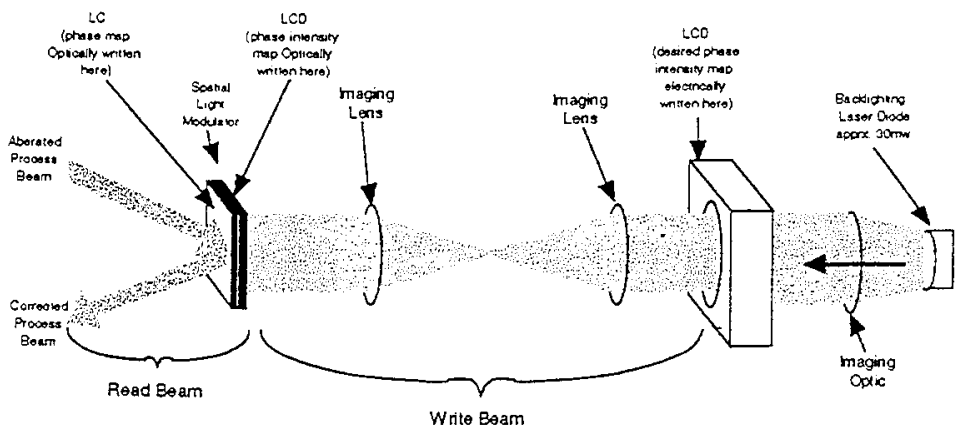

Figure 2. Operational configuration for optically addressed liquid crystal spatial light modulator 


\section{PHASE RECONSTRUCTION METHODS}

Accurately controlling wavefront phase with a high-spatial-resolution wavefront correction device requires a high-spatialresolution wavefront measurement capability. This can be provided with a standard Shack-Hartmann wavefront sensor using available high-density lenslet arrays. However, computational requirements for traditional matrix-vector multiply wavefront reconstruction algorithms using slope data from a Shack-Hartmann (or other wavefront slope) sensor scale as $\mathrm{N}^{2}$ where $\mathrm{N}$ is the number of phase points. For large $\mathrm{N}$, greater than $\sim 10^{3}$ this begins to become impractical. Since the slope measurement of the Shack-Hartmann sensor can be represented by a spatial filtering operation, an inverse filter can be designed to directly recover the wavefront from the slope data. ${ }^{12}$ This allows the reconstruction process to be implemented as a $2 D$ convolution operation using FFT's, which scale as $\mathrm{Nlog}_{2} \mathrm{~N}$. We have used computer simulations to demonstrate that for 4096 phase points the FFT-based algorithm is $\sim 30$ times faster than a matrix multiply (Figure 3 ). A similar approach has been utilized by Chanteloup et al with a shearing interferometer as the wavefront slope sensor.

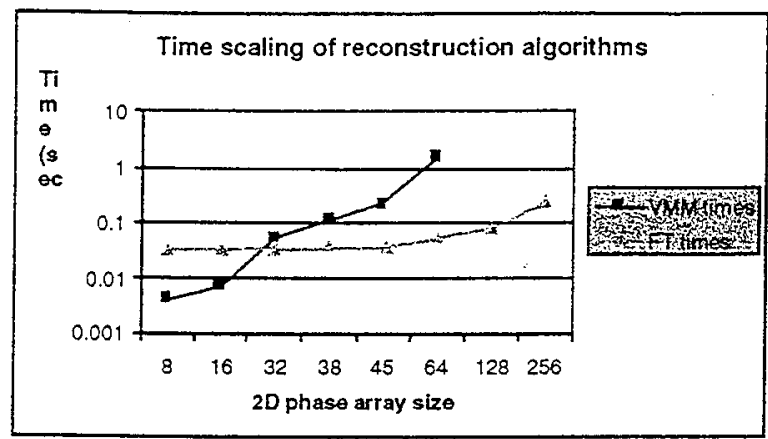

Times for single R10000 CPU in View

Figure 3. Computational scaling for phase reconstruction algorithms

We have used the FFT-based reconstruction method in lab experiments using the test-bed described in Figure 5 with a ShackHartmann sensor with $>2500$ subapertures and using a $1 \mathrm{Kx} 1 \mathrm{~K}$-pixel camera. To verify that the algorithm would accurately represent the pattern generated, a checkered phase pattern was produced on the LC SLM and Shack-Hartmann image data was recorded (Figure 4a). The wavefront phase was then reconstructed using the FFT-based method (Figure 4b). The resulting phase reconstruction accurately depicted the applied checkered phase pattern. Additional tests were performed using different phase patterns with each reconstruction accurately representing the applied pattern.

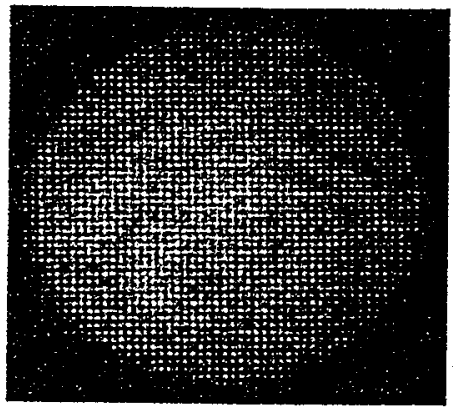

Figure 4a High-Resolution Hartmann Sensor

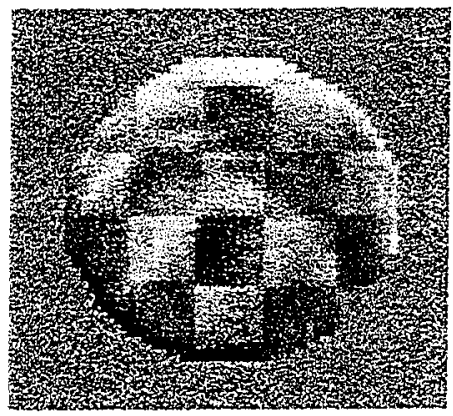

Figure 4b Reconstructed Wavefront using FFT's

\section{SLM EXPERIMENTAL TEST BED}

A test-bed, shown schematically in Figure 5, has been assembled to allow the evaluation of SLM devices for high-spatialresolution spatial wavefront control. This test-bed provides the capability to place both low-order and high-order aberrations 
onto a beam, to detect these aberrations with a bigh-spatial-resolution Shack-Hartmann sensor, and to correct these phase aberrations using the OA LC SLM. This process is currently implemented using multiple computer systems in a human-inthe-loop closed-loop operation.

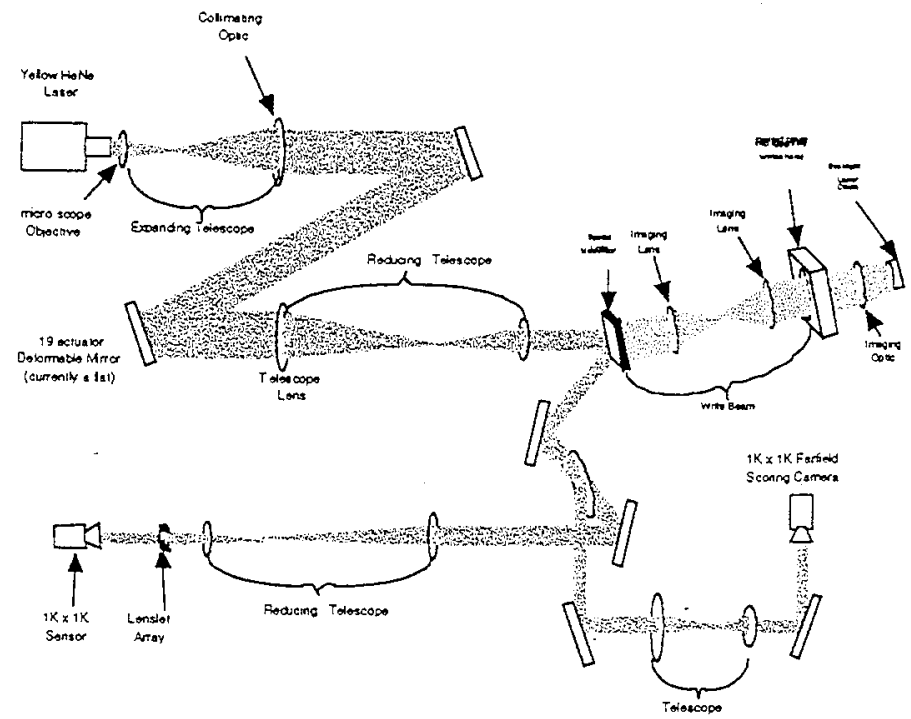

Figure 5. High-resolution wavefront control test-bed schematic

\section{EXPERIMENTAL RESULTS}

An initial set of experiments has been completed using the Jenoptik OA LC SLM. In these experiments, an aberration was placed into the main beam path (Figure 6a) and the resulting high-resolution Shack-Hartmann image was processed using the FFT-based method to reconstruct the phase (Figure $6 \mathrm{~b}$ ). From the reconstructed phase an appropriate correction image was generated and applied via the SLM. The applied correction produced a significantly improved far field spot (Figure $6 \mathrm{c}$ ). The corrected wavefront was reanalyzed with the same methods and a significant improvement was observed in the reconstructed phase image (Figure 6d).

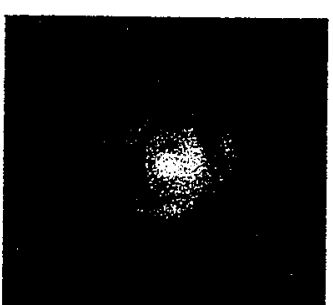

Figure 6a Aberrated far-field

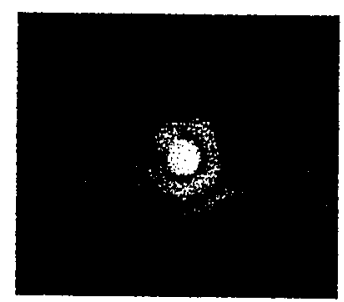

Flgure 6e Corrected far-field

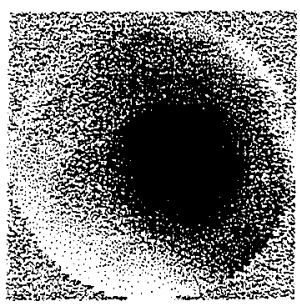

Figure 6b Aberrated Wavetront

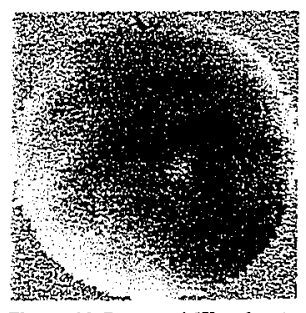

Figure 6d Corrected Wavefront

Figure 6. Initial High-resolution closed-loop wavefront control experimental results. 
Similar experiments utilizing the Hamamatsu AO_LC SLM are currently underway in the SLM test-bed with some modifications to improve the collimation of the write-beam from the back-light diode and thereby improve the correlation between the image pattern generated on the LCD and the phase correction produced by the SLM.

\section{FUTURE PLANS}

Design for the introduction of an OA LC SLM into a high-powered short-pulse laser is also currently in progress. The OA LC SLM will be used to correct high-spatial-frequency phase errors in the front end of the Petawatt Short-Pulsed Laser. ${ }^{\text {ts }}$ In this experiment, we will be testing the ability of the SLM to correct for high-spatial-frequency errors and the effects on transport of the correction through the spatial filters of the system. The OA LC SLM will also be inserted into the system in two additional locations that have a higher beam fluence to evaluate the performance in high-power conditions and to determine the damage threshold.

\section{ACKNOWLEDGEMENTS}

The authors would like to acknowledge the technical assistance of J. An, C. Carrano, R. Hurd, and R. Sawvel. Work performed under the auspices of the U.S. Department of Energy by the Lawrence Livermore National Laboratory under Contract number W 7405-Eng-48.

\section{REFERENCES}

1. K. Freischland and C. L. Koliopoulos, "Wavefront Reconstruction from Noisy Slope or Difference Data Using the Discrete Fourier Transform", Proc. SPIE 551, 1985.

2. D. C. Ghiglia and L. A. Romero, "Robust two-dimensional weighted and unweighted phase unwrapping that uses fast transforms and iterative methods", J. Opt. Soc. Am, A 11, pp. 107-117, 1994.

3. J. C. Chanteloup, F. Druon, M. Nantel, A. Maksimchuk, G. Mourou, "Single-shot wave-front measurements of highintensity ultrashort laser pulses with a three-wave interferometer", Opt. Lett., 23, pp. 621-623, 1998.

4. M. D. Perry, D. Pennington, B. C. Stuart, G. Tietbohl, J. A. Britten, C. Brown, S. Herman, B. Golick, M. Kartz, J. Miller, H. T. Powell, M. Vergino, and V. Yanovsky, "Petawatt laser pulses", Opt. Lett., 24, pp. 160-162, 1999.

5. D. M. Pennington, C. G. Brown, M. Kartz, M. Landon, M. D. Perry, G. Tietbohl, "Production of high intesity laser pulses with adaptive optics", International Workshop on Adaptive Optics for Industry and Medicine, Durham, England, July 12-16, in press, 1999. 


\title{
Chapter 5 - Fast Fourier and Wavelet Transforms for Wavefront Reconstruction in Adaptive Optics
}

\author{
Farid U. Dowla, James M. Brase, Scot S. Olivier, and Charles A. Thompson \\ Lawrence Livermore National Laboratory, Livermore, California 94551
}

\begin{abstract}
Wavefront reconstruction techniques using the least-squares estimators are computationally quite expensive. We compare wavelet and Fourier transforms techniques in addressing the computation issues of wavefront reconstruction in adaptive optics. It is shown that because the Fourier approach is not simply a numerical approximation technique unlike the wavelet method, the Fourier approach might have advantages in terms of numerical accuracy. To optimize the wavelet method, a statistical study might be necessary to use the best basis functions or "approximation tree."
\end{abstract}

Keywords: Novel algorithms and architectures, adaptive optics, wavefront reconstruction, fast algorithms, real-time imaging, wavefront control, phase reconstruction, FFT methods, wavelets, MEMS.

\section{INTRODUCTION}

Wavefront reconstruction techniques for real-time imaging applications using adaptive optics are computationally intensive. As we approach implementation of adaptive optics systems with thousands of actuators employing micro-electromechanical systems (MEMS), the use of efficient high-performance algorithms for wavefront reconstruction will be of significant importance. In this study we have evaluated various fast wavelet and Fourier methods in order to determine both the speed and robustness of wavefront reconstruction algorithms for real-time imaging in large scale adaptive optics systems.

We developed fast signal processing algorithms for wavefront reconstruction. In particular, we have studied both fast discrete wavelet (DWT) and fast Fourier methods (FFT) to determine the performance of these algorithms in wavefront phase reconstruction. The wavelet method is of particular interest because the order of the computation for the 1-D DWT is only $\mathrm{O}(\mathrm{N})$ as opposed to $\mathrm{O}(\mathrm{N} \log \mathrm{N})$ for the 1-D FFT. The usefulness of the wavelets in terms of reduced number of computations is enhanced by the fact that the transform coefficients can be truncated significantly; i.e. transformed into sparse expansions. This energy compaction property of the 2-D wavelet transforms can be exploited fully in the wavefront reconstruction algorithm in adaptive optics. Since there are many "good wavelets" or basis functions, in this study we compare the performance for wavelets for fast and robust wavefront reconstruction.

In section 2 we first derive the Fourier approach and show that this approach is really an analytical alternative to the true solution. On the other hand, in section 3 we show that the wavelet method is strictly a numerical approximation to the least square technique. We illustrate our results with the wavelet scheme, and summarize our conclusions in section 4.

\section{WAVEFRONT RECONSTRUCTION USING THE FFT}

If we sample a wavefront on a regular $2 \mathrm{D}$ grid we have an array of phase values $\varphi_{m, n}$ where $0 \leq m \leq M-1$ and $0 \leq n \leq N-1$. The phase differences $\Delta_{m, n}^{x}$ and $\Delta_{m, n}^{y}$ are then given by

$$
\begin{aligned}
\Delta_{m, n}^{x} & =\varphi_{m+1, n}-\varphi_{m, n} \\
\Delta_{m, n}^{y} & =\varphi_{m, n+1}-\varphi_{m, n}
\end{aligned}
$$

We can write the phase differences as a matrix equation

$$
\vec{\Delta}=A \vec{\varphi}
$$


where $\vec{\Delta}$ is a column vector containing the $\mathrm{x}$ and $\mathrm{y}$ phase differences, $\vec{\varphi}$ is a column vector containing the phase values, and $A$ is a finite difference matrix. The least-squares solution for $\vec{\varphi}$, given a slope measurement vector $\vec{\Delta}$, is given by solving the normal equations

$$
A^{T} A \vec{\varphi}=A^{T} \vec{\Delta}
$$

In most adaptive optics systems the normal equations are solved directly using singular-value decomposition to give the phase estimate

$$
\hat{\varphi}=\left(A^{T} A\right)^{-1} A^{T} \vec{\Delta}
$$

This solution requires $(M N)^{2}$ operations to implement the real-time phase reconstructor, assuming that the matrix $\left(A^{T} A\right)^{-1} A^{T}$ is pre-computed.

For large-scale systems with thousands of phase sample points we need a more computationally efficient approach. The normal equations can be written in the form [3]:

$$
\left(\varphi_{m+1, n}-2 \varphi_{m, n}+\varphi_{m-1, n}\right)+\left(\varphi_{m, n+1}-2 \varphi_{m, n}+\varphi_{m, n-1}\right)=\rho_{m, n}
$$

where

$$
\rho_{m, n}=\left(\Delta_{m, n}^{x}-\Delta_{m-1, n}^{x}\right)+\left(\Delta_{m, n}^{y}-\Delta_{m, n-1}^{y}\right)
$$

Note that this is a discretization of Poisson's equation $\frac{\partial^{2} \varphi}{\partial x^{2}}+\frac{\partial^{2} \varphi}{\partial y^{2}}=\rho$. The phase $\varphi_{m, n}$ can be represented by its discrete Fourier coefficients as

$$
\varphi_{m, n}=\frac{1}{N} \sum_{p, q} \tilde{\varphi}_{p, q} e^{i \frac{2 \pi}{N}(p m+q n)}
$$

where we have assumed that $M=N$, i.e. the phase sample grid is square. We also have

$$
\varphi_{m+1, n}=\frac{1}{N} \sum_{p, q} \tilde{\varphi}_{p, q} e^{i \frac{2 \pi p}{N}} e^{i \frac{2 \pi}{N}(p m+q n)}
$$

Now we can write the normal equations in terms of the Fourier coefficients as

$$
\begin{aligned}
& \sum_{p, q} \tilde{\varphi}_{p, q} e^{i \frac{2 \pi}{N}(p m+q n)}\left(e^{i \frac{2 \pi p}{N}}-2+e^{-i \frac{2 \pi p}{N}}\right)+\sum_{p, q} \tilde{\varphi}_{p, q} e^{i \frac{2 \pi}{N}(p m+q n)}\left(e^{i \frac{2 \pi q}{N}}-2+e^{-i \frac{2 \pi q}{N}}\right) \\
& =\sum_{p, q} \widetilde{\rho}_{p, q} e^{i \frac{2 \pi}{N}(p m+q n)}
\end{aligned}
$$

where $\widetilde{\rho}_{p, q}$ are the Fourier coefficients of $\rho_{m, n}$. For spatial frequency $\mathrm{p}, \mathrm{q}$ we can solve directly for $\varphi_{p, q}$ as

$$
\widetilde{\varphi}_{p, q}=\frac{\tilde{\rho}_{p, q}}{2\left(\cos \frac{2 \pi p}{N}+\cos \frac{2 \pi q}{N}-2\right)}
$$


Now we need to write $\widetilde{\rho}_{p, q}$ in terms of the measurements $\Delta_{m, n}$

$$
\tilde{\rho}_{p, q}=\mathfrak{I}\left\{\Delta_{m, n}^{x}\right\}-\mathfrak{S}\left\{\Delta_{m-1, n}^{x}\right\}+\mathfrak{I}\left\{\Delta_{m, n}^{y}\right\}-\mathfrak{I}\left\{\Delta_{m, n-1}^{y}\right\}
$$

where $\mathfrak{I}\{f\}$ is the Fourier transform of $f$. Since $\mathfrak{I}\left\{\Delta_{m-1, n}^{x}\right\}=e^{i \frac{2 \pi p}{N}} \widetilde{\Delta}_{p, q}^{x}$ we have

$$
\tilde{\rho}_{p, q}=\left(1-e^{-i \frac{2 \pi p}{N}}\right) \tilde{\Delta}_{p, q}^{x}+\left(1-e^{-i \frac{2 \pi q}{N}}\right) \tilde{\Delta}_{p, q}^{y}
$$

Then we can write the Fourier coefficients of the phase estimate as the sum of two spatial filter operations

$$
\tilde{\varphi}_{p, q}=H_{p, q}^{x} \tilde{\Delta}_{p, q}^{x}+H_{p, q}^{y} \widetilde{\Delta}_{p, q}^{y}
$$

where the filters are given by

$$
\begin{aligned}
& H_{p, q}^{x}=\frac{e^{-i \frac{2 \pi p}{N}}-1}{4\left(\sin ^{2} \frac{\pi p}{N}+\sin ^{2} \frac{\pi q}{N}\right)} \\
& H_{p, q}^{y}=\frac{e^{-i \frac{2 \pi q}{N}}-1}{4\left(\sin ^{2} \frac{\pi p}{N}+\sin ^{2} \frac{\pi q}{N}\right)}
\end{aligned}
$$

The algorithm for the Fourier wavefront reconstructor is given by

1. Compute the Fourier transforms of the phase differences from the wavefront sensor, $\tilde{\Delta}_{p, q}^{x}, \widetilde{\Delta}_{p, q}^{y}$,

2. Apply the spatial filters $H_{p, q}^{x}, H_{p, q}^{y}$ to get $\widetilde{\varphi}_{p, q}$

3. Inverse Fourier transform to get $\varphi_{m, n}$

If $\mathrm{N}$ is a power of two the spatial filter operations can be implemented with FFT's. The computational requirements then scale as $O\left(N^{2} \log _{2} N\right)$ rather than as $O\left(N^{4}\right)$ in the direct vector-matrix multiplication approach.

\section{WAVEFRONT RECONSTRUCTION USING WAVELETS}

Although wavelets are well known for their signal and image compression properties [2,5,6], a less well-known use of the wavelet transform is in obtaining a fast approximate numerical solution [3] for a system of linear equations. As discussed in the previous section, this implies wavelets can be used in the adaptive optics wavefront reconstruction problem. The matrix operator, in solving a system of linear equations, can be thought of as a two-dimensional image. Taking advantage of the energy compaction property of the two-dimensional wavelet transform, we can expect a large fraction of the wavelet 
coefficients to be small and negligible. Hence, the linear system to be solved, in the transform domain, is a sparse system (mostly zero coefficients). To be more specific, consider solving a set of linear equations of the form:

$$
C x=b
$$

The two-dimensional wavelet transform of the control matrix, $\mathrm{C}$, and the vector $\mathrm{b}$ are denoted by:

$$
\begin{gathered}
\widetilde{C}=W C W^{T} \\
\tilde{b}=W b
\end{gathered}
$$

where $W$ represents the kernel of the wavelet transform operator. Now we can solve for

$$
\widetilde{C} \tilde{x}=\tilde{b}
$$

where $\tilde{x}$ represents the wavelet transform of the solution vector. To obtain the solution $x$, we take a final inverse of the orthogonal wavelet transform:

$$
x=W^{T} x
$$

The efficiency of the wavelet transform in solving a set of linear equations will ultimately depend both on the sparseness of the matrix $\widetilde{C}$ and on the particular wavelet transform approximation algorithm. For example, consider the 2-D wavelet transform depicted in Figure 1.

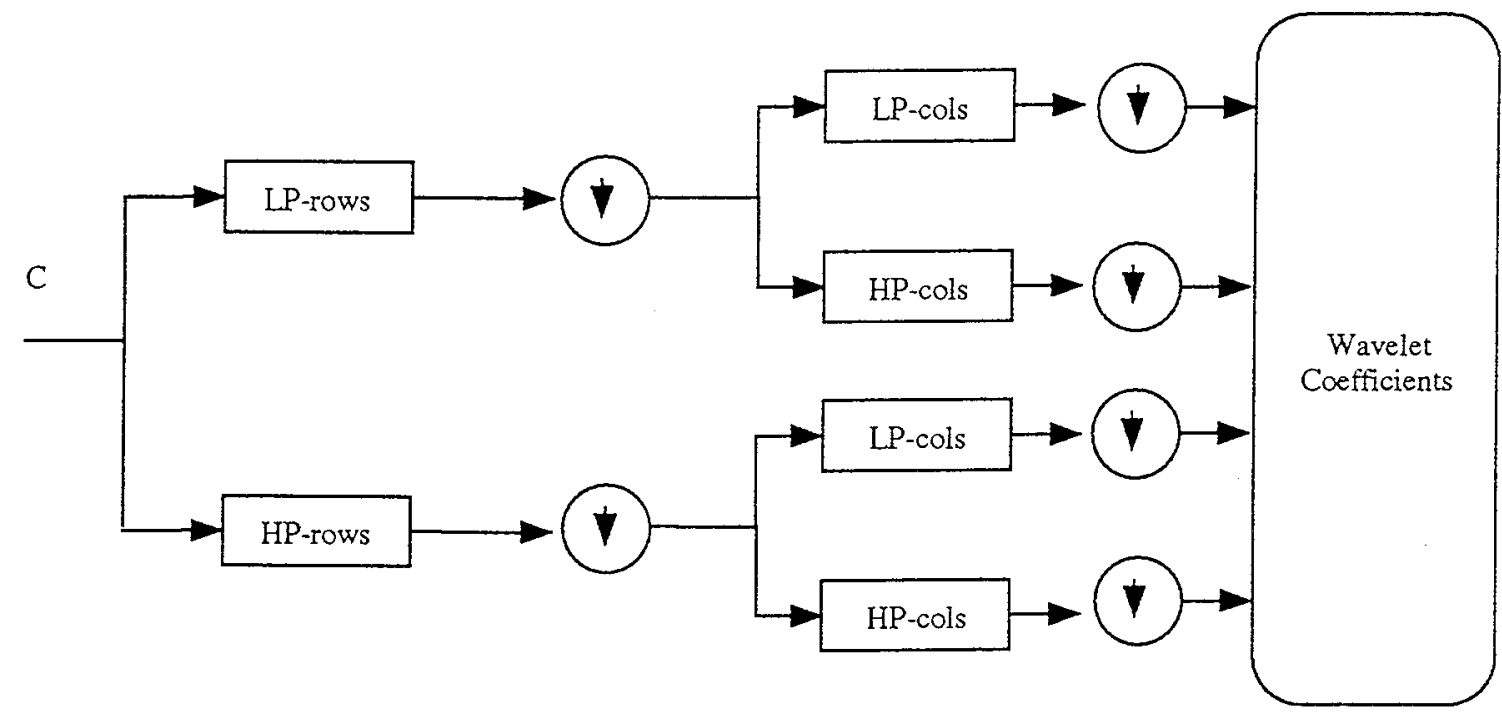

Figure 1: A block diagram of the forward wavelet transform for two-dimensional waveforms or images. Note that for the inverse transform, simply replace downsampling (down-arrows) by upsampling (up-arrows) and right-arrows by left arrows.

The accuracy of the numerical approximation and the number of computation will depend on the number of decomposition steps and will further depend on the filterbanks "tree" or path employed for the transformation [5]. There are also a number of different approximation trees that can be chosen from; i.e. the exact path of lowpass and highpass filters. We studied lowpass, highpass, and both lowpass and highpass approximations for adaptive optics wavefront reconstruction. In our implementation scheme, we found that the lowpass wavelet coefficients were generally more useful. The results in this paper are based on the double lowpass wavelet approximation coefficients. The inverse wavelet transform is very similar to the forward transform shown in Figure 1, except that the down-sampling by two (down-arrows) are replaced by up-sampling 
(placing zeros in between data samples) and the right-arrows are to be replaced by left arrows. Finally, the set of filter coefficients used to compute the wavelet transforms and the inverse wavelet transforms is shown in Figure 2.
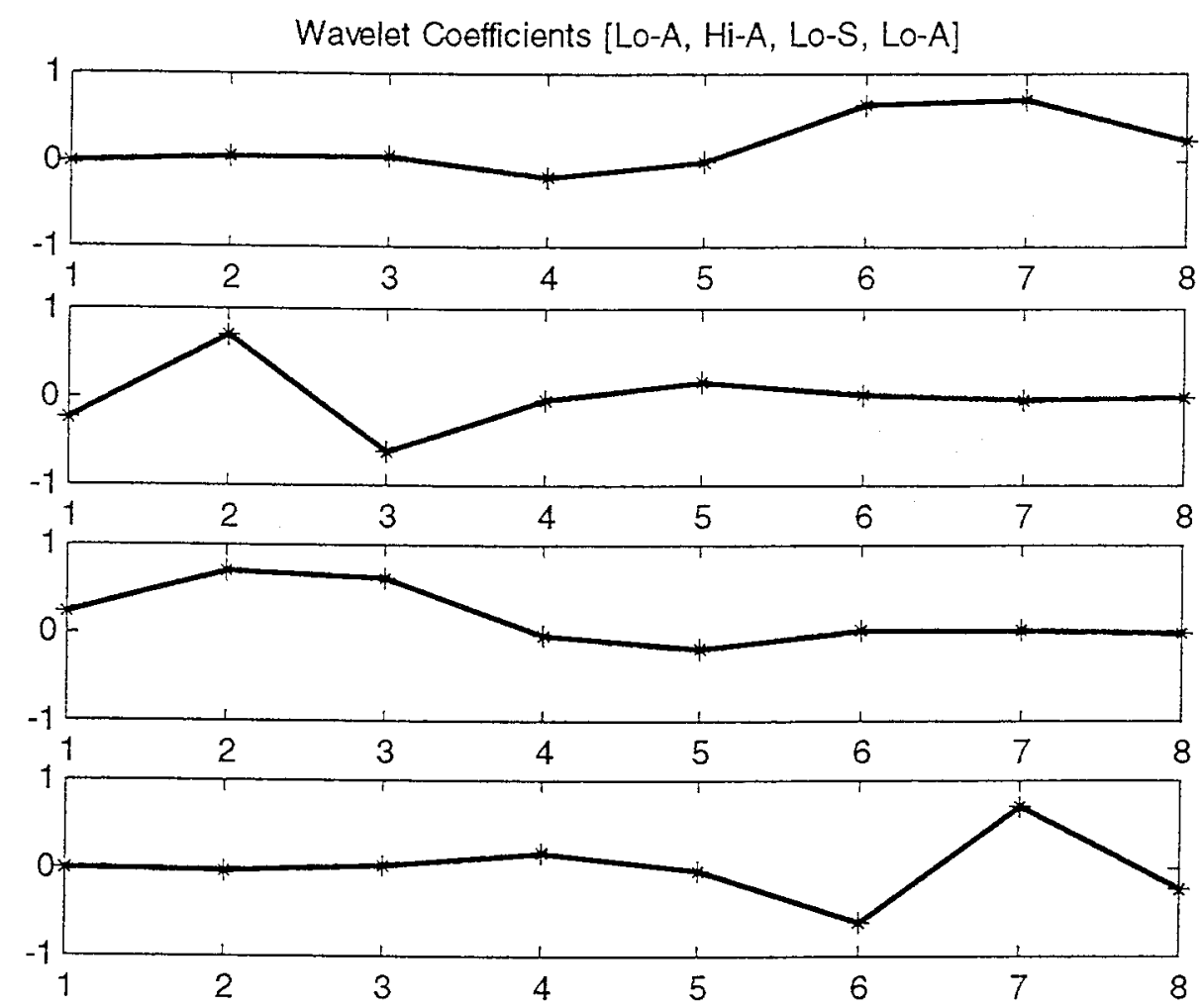

Figure 2: Plots of the typical wavelet transform filter coefficients: lowpass analysis, highpass analysis, lowpass reconstruction, and highpass resconstruction filters.

The output of the filter coefficients shown in Figure 2 is computed by:

$$
y(k)=\sum_{i=1}^{L} b(i) x(k-i),
$$

where filter coefficients, $b(i)$, are shown in the plot above, and $x(k)$ and $y(k)$ represents input and output signals, respectively.

In summary, the algorithm can be described as follows:

Step 1: Compute the wavelet matrix approximation for a single stage wavelet transform. This step is precomputed just once, and depends on the system.

Step 2: This step is repeated multiple times:

2.1 Compute the wavelet transform of the input vector;

2.2 Compute multiplication in the wavelet domain;

2.3 Reconstruct the vector approximation by taking the inverse wavelet transform.

Note that because the wavelet transform basis has only a small number of terms (localized) and because of the downsampling operations, it can be shown that the computation of the wavelet transform is $O(M)$ in 1-D and $O\left(M^{2}\right)$ in 2-D, where $\mathrm{M}$ is the dimension of the input. The computational requirements for the wavelet wavefront reconstruction approach then scales 
as $O\left(N^{3}\right)$ [2] rather than as $O\left(N^{4}\right)$ in the direct vector-matrix multiply approach. A note of caution: although the wavelet transform is numerically most attractive, it is important to point out that this approach is an approximation and its usefulness will ultimately depend on how well it does in terms of numerical accuracy. In the next section we present results from applying the wavelet reconstruction method of real wavefront data.

\section{NUMERICAL EXAMPLES USING WAVELETS}

In section 4.1 we first illustrate the wavelet approximation scheme using a simple tractable numerical example. Performance of the approximation on real data is illustrated in sections 4.2 and 4.3. Finally, comments on areas of care required for using this method are included.

\subsection{A simple numerical illustration}

For the sake of clarity we first consider simple numerical example on matrix-vector multiplication using the wavelet transform scheme. Consider a $(8 \times 8)$ matrix $C$, and a $(8 \times 1)$ column vector $x$ shown below:

$$
C=\left[\begin{array}{cccccccc}
62 & 2 & 3 & 61 & 60 & 6 & 7 & 57 \\
9 & 55 & 54 & 12 & 13 & 51 & 50 & 16 \\
17 & 47 & 46 & 20 & 21 & 43 & 42 & 24 \\
40 & 26 & 27 & 37 & 36 & 30 & 31 & 33 \\
32 & 34 & 35 & 29 & 28 & 38 & 39 & 25 \\
41 & 23 & 22 & 44 & 45 & 19 & 18 & 48 \\
49 & 15 & 14 & 52 & 53 & 11 & 10 & 56 \\
8 & 58 & 59 & 5 & 4 & 62 & 63 & 1
\end{array}\right], x=\left[\begin{array}{l}
1 \\
2 \\
3 \\
4 \\
5 \\
6 \\
7 \\
8
\end{array}\right]
$$

The numerical solutions to the direct matrix-vector multiplication and the wavelet approximation are respectively given by

$$
\begin{aligned}
& C x=[1162,1178,1178,1162,1162,1178,1178,1162]^{T} \\
& w^{-1}[\tilde{C} \tilde{x}]=[1170,1170,1170,1170,1170,1170,1170,1170]^{T}
\end{aligned}
$$

Note that in the wavelet approximation method, we used the $2^{\text {nd }}$ order Daubechies wavelet coefficients with two consecutive lowpass (or smooth approximation) filtering and downsampling of the rows and the columns. The solution therefore represents a smooth approximation of the exact solution. In general, this is an important point about the wavelet approximation method on real data: the approximation quality depends on the exact decomposition tree (i.e. the path of lowpass and highpass approximations) and on determining how well a particular tree is able to represent the matrix $\mathrm{C}$ and therefore also the solution vector $C x$.

\subsection{Numerical examples with real data}

An example of the AO control matrix is shown as an image in Figure 3. To illustrate the approximation with a discernable plot, we show part of the reconstruction results in Figure 4, and compare this wavelet approximation solution with the conventional reconstruction technique. In this particular example, we used a constant signal for $x$, the slope measurement vector. 


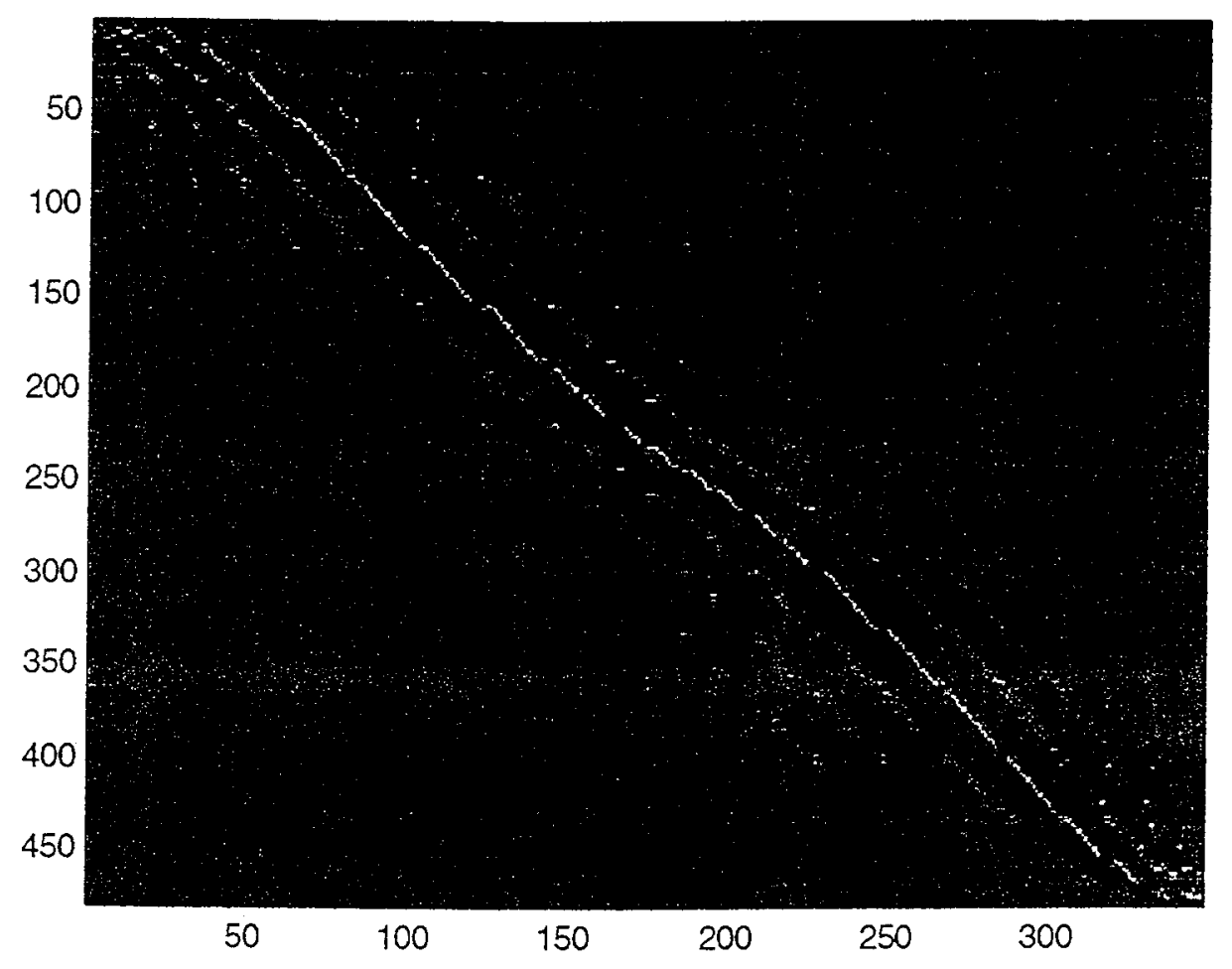

Figure 3: The AO control matrix represented as an image

Although the wavelet transform technique is computationally efficient, through the course of this study we discovered that the approximation quality does depend on the form of system matrix, $A$, and on the multiplying vector, $x$. Furthermore, the exact lowpass-highpass combinations used in the approximation algorithm is also quite important. Our conclusion is that to use the wavelet transform technique successfully, we would need to first carefully characterize the statistical properties of the distorted wavefronts and then select the best "basis" (the lowpass/highpass tree). A significant amount of work has been performed in wavelet packets [6] that could be used for optimal performance. We plan to evaluate the performance of this approximation scheme in a full adaptive optics system simulation mode.

In Figures 4-a, 4-b, and 4-c we compare the wavelet results with the least-squares results for multiplying with a constant vector, $\Delta$, for one, two, and three stage wavelet approximations. Due to the iterative smoothing effects, although a multistage wavelet approximation is more efficient numerically, there is considerable loss in the high-frequency components of the solution. Whether in practice this loss of high frequency components is desirable or not, we will not know until the method is used in a complete working system. However, we have summarized the numerical efficiency versus numerical loss in Figure 5. The numerical loss is calculated using the rms loss defined as:

$$
\text { Normalized RMS Loss }=10 \log _{10}\left[\frac{\left\|X_{\text {Wavelet }}-X_{L S}\right\|}{\left\|X_{L S}\right\|}\right]
$$

A normalized rms loss of $-3 \mathrm{~dB}$ seems apparently acceptable, while a loss of $0 \mathrm{~dB}$ entails a significant degree of smoothing. From the results summarized in Figure 5 we conclude that the wavelet approximation scheme can reduce the number of computation by an order of magnitude without severely encountering loss in numerical accuracy. 


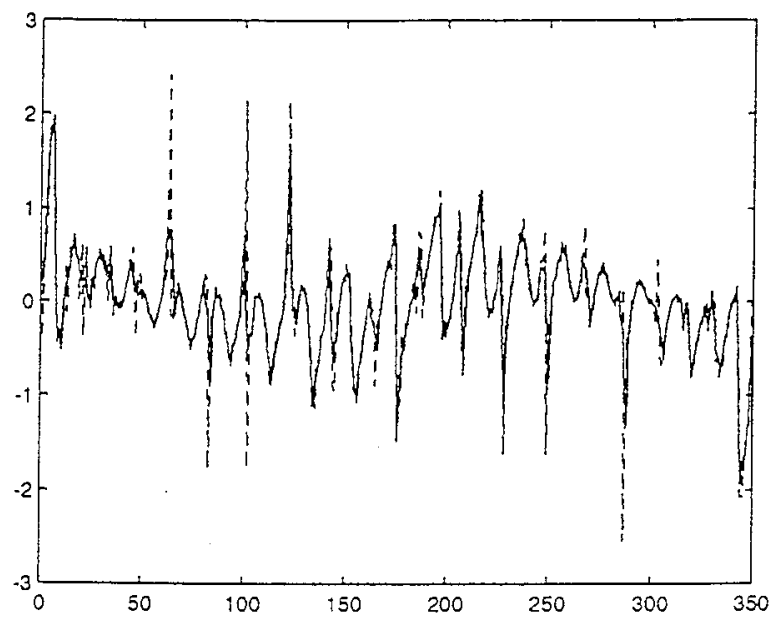

Figure 4-a: A comparison of the wavelet approximation solution (solid) with the least-squares (LS) solution (dashed). Only one-stage, a single lowpass analysis and synthesis stages were, employed in this implementation.

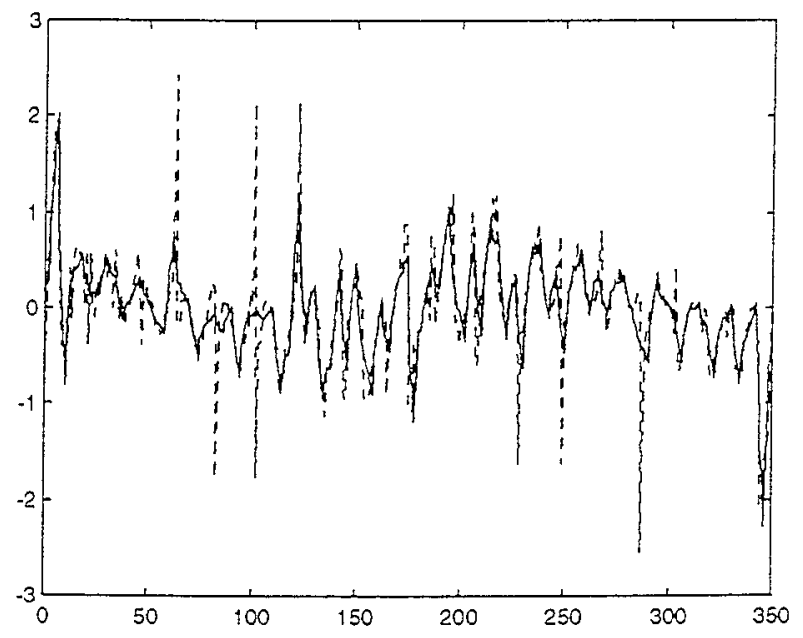

Figure 4-b: A comparison of the wavelet approximation solution (solid) with the least-squares (LS) solution (dashed). A two-stage, two lowpass analysis and synthesis stages were, employed in this implementation. 


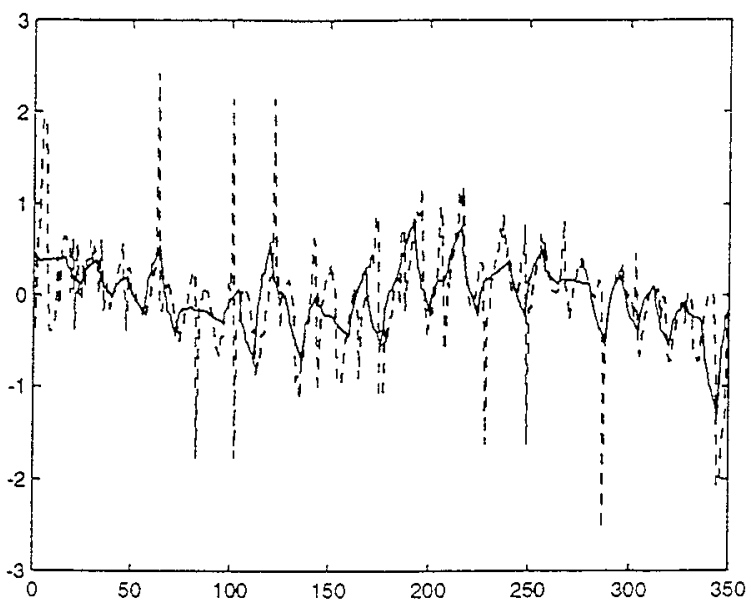

Figure 4-c: A comparison of the wavelet approximation solution (solid) with the least-squares (LS) solution (dashed). A three-stage, three lowpass analysis and synthesis stages were, employed in this implementation.

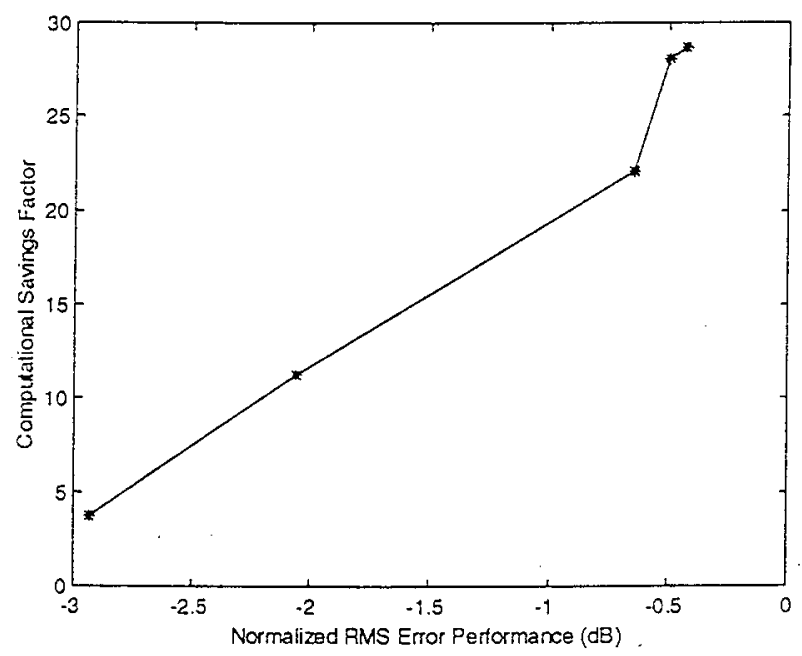

Figure 5: Results summarizing computational saving (wrt standard LS approach) versus numerical loss in using the wavelet approximation technique.

Visually a $-3 \mathrm{~dB}$ loss (see Figure 4-a) appeared acceptable. While a $0 \mathrm{~dB}$ loss introduced a significant degree of smoothing into the solution. Results of this study indicate that computation can be decreased by an order of magnitude using the wavelet scheme.

\section{SUMMARY AND CONCLUSIONS}

In this paper we discussed how the FFT and the wavelet transform methods could be used to speed up computation in adaptive optics wavefront reconstruction. We were particularly interested in comparing wavelet method with the FFI technique. As discussed in sections 2 and 3 , we find that the wavelet approximation may or may not be accurate enough, only application of the scheme on a real system can determine this; i.e. a full scale simulation study can determine the performance in terms numerical accuracy; we plan to do this in the near future. The accuracy of the approximation depends 
on the particular vector and matrix properties, and on the choice of the wavelet tree used to decompose the tree. A statistical study will be needed to select the optimal wavelet decomposition. However, preliminary results from this study indicate that we can reduce the number of computation by an order of magnitude using the wavelet approximation scheme. We also observed that the type of wavelet basis function used does not seem to make a significant difference in the numerical results. A low order basis function, like the Daubechies $2^{\text {nd }}$ order coefficients, seems to be adequate with real data. On the other hand, as discussed in section 2, the Fourier approach is much less of a numerical approximation, and more of an analytical

solution until the application of the FFT. Hence, the FFT approach might be more suitable to minimize loss in the numerical accuracy.

\section{ACKNOWLEDGEMENTS}

Work performed under the auspices of the U.S. Department of Energy by Lawrence Livermore National Laboratory under contract W-7405-ENG-48.

\section{REFERENCES}

1. Anant, K. S., F. U. Dowla, G. H. Rodrigue. "Vector quantization of ECG wavelet coefficients", EEE Signal Processing Letters, pp. 1-4, July 1995.

2. Bowman, K. and W, Rhodes, "Application of wavelets to wavefront reconstruction in optical systems", SPIE Vol. 3126, pp. 288-294, August 1997.

3. Freischlad, K and C. Koliopoulos, "Wavefront reconstruction from Noisy or Difference Data Using the Discrete Fourier Transform," SPIE Vol. 551, pp. 74-80, 1985.

4. Hunt, B. R., "Matrix formulation of the reconstruction of phase values from phase differences," J. Opt. Soc. Am., Vol. 69, No. 3, pp. 393-399, March 1979.

5. Vaidyanathan, P. P. "Multirate Systems and Filterbanks," Prentice-Hall, 1993.

6. Vetterli, M. and J. Kovacevic, "Wavelets and Subband Coding, Prentice-Hall, 1995. 


\title{
Chapter 6 - Performance of a high-resolution wavefront control system using a liquid crystal spatial light modulator
}

\author{
C. A. Thompson*, M. W. Kartz, S. S. Olivier, J. M. Brase, C. J. Carrano, \\ D. Lande, B. J. Bauman, R. M. Sawvel, D. A. Silva \\ Lawrence Livermore National Laboratory ${ }^{\circ}$ Livermore, California, 94550
}

\begin{abstract}
We have developed a high-resolution wavefront control system based on an optically addressed nematic liquid crystal spatial light modulator with several hundred thousand phase control points, a Shack-Hartmann wavefront sensor with two thousand subapertures, and an efficient reconstruction algorithm using Fourier transform techniques. We present quantitative results of experiments to characterize the performance of this system.
\end{abstract}

Keywords: Adaptive Optics, high-resolution wavefront control, spatial light modulator

\section{INTRODUCTION}

Recent technological developments, mainly driven by the video projection industry, have led to the fabrication of new highresolution wavefront control devices, such as the liquid crystal or micro-electro-mechanical spatial light modulators. These new high-resolution devices may, in turn, enable new high-performance wavefront control applications. For many years, adaptive optics have been based on thin deformable glass mirrors utilizing piezoelectric or electrostrictive actuators. These mirrors have been used to remove time-varying aberrations in optical systems, such as high-power laser systems or telescopes imaging through atmospheric turbulence. In the case of high-power lasers, adaptive optics systems have successfully improved beam quality, but bave often revealed high-spatial-frequency aberrations, beyond the capability of deformable mirrors to remove. In addition, the manufacturing errors of deformable mirrors have often introduced high-spatial-frequency wavefront errors.

The largest deformable mirrors currently available have approximately one thousand actuators and cost approximately $\$ 1 \mathrm{M}$. This cost scaling of $\sim \$ 1000$ per actuator makes deformable mirrors cost prohibitive for many applications that require highspatial-resolution wavefront control. Therefore, a new approach using a different wavefront control technology is needed. One approach is the use of liquid crystal (LC) spatial light modulator (SLM) technology for controlling the phase of linearly polarized light. Current LC SLM technology provides high-spatial-resolution wavefront control, with hundreds of thousands of degrees of freedom, more than two orders of magnitude greater than the largest deformable mirrors currently made. Furthermore, even with the increased spatial resolution, the cost of these devices is nearly two orders of magnitude less than the cost of the largest deformable mirror. Thus, LC SLM devices have the potential to create a paradigm shift of four orders of magnitude in the cost per phase control point for advanced adaptive optics. However, in order to fulfill this promise, LC SLM technology must be demonstrated to achieve the required optical performance in an integrated system configuration. In this paper, we will present quantitative results for the optical performance of a LC SLM device in an adaptive optics test-bed.

\section{SLM TECHNOLOGY}

The LC SLM devices utilized in the investigations described in this paper are a type of optically-addressed (OA) parallel aligned nematic LC SLM, currently available from both Hamamatsu and Jenoptik. This paper will primarily address the tests of the Hamamatsu device, results from the Jenoptik device test have been presented previously'. The LC SLM devices are capable of phase corrections slightly greater than one wave at visible and near-infra-red wavelengths. An OA LC SLM consists of a thin-film sandwich structure, shown in Figure 1, with an amorphous silicon ( $\alpha-S i: H)$ photo-semiconductor, a parallel aligned nematic liquid crystal, a dielectric mirror, and a pair of transparent electrodes on glass substrates. The voltage applied to the electrodes is divided between photo-semiconductor and liquid crystal layers, depending on the illumination intensity, thereby enabling $2 \mathrm{D}$-image modulation of the refractive index of the LC.

"Correspondence: L-258, 7000 East Avenue, Livermore, CA, 94550; Email: charlest@llnl.gov.

This work was performed under the auspices of the U.S. Department of Energy by University of California, Lawrence Livermore National Laboratory under contract No. W-7405-Eng-48. 
To activate the OA LC SLM, the amorphous silicon ( $\alpha-\mathrm{Si}: \mathrm{H})$ layer must be exposed to an image pattern, preferably illuminated between $670-690 \mathrm{~nm}$ where the $(\alpha-\mathrm{Si}: \mathrm{H})$ layer is most sensitive. In the state where no pattern is incident on the device, the impedance of the $\alpha$-Si:H layer is very high, irrespective of an applied voltage. With an applied voltage, an image pattern projected onto the $\alpha$-Si:H layer reduces its impedance in proportion to the light intensity in that region, thereby increasing the voltage applied to the liquid crystal. The increased voltage causes the LC molecules to rotate in the direction of the applied field, thus modulating the phase of the incident light. The maximum phase shift is obtained when the polarization of readout light is parallel to the axis of the liquid crystal molecules. When the polarization of readout light is perpendicular to the axis of the liquid crystal molecules the phase shift is negligible. A dielectric layer is manufactured into the device to increase device reflectivity. Incident light, therefore, passes through the LC layer twice (on incidence and upon reflection from the dielectric layer).
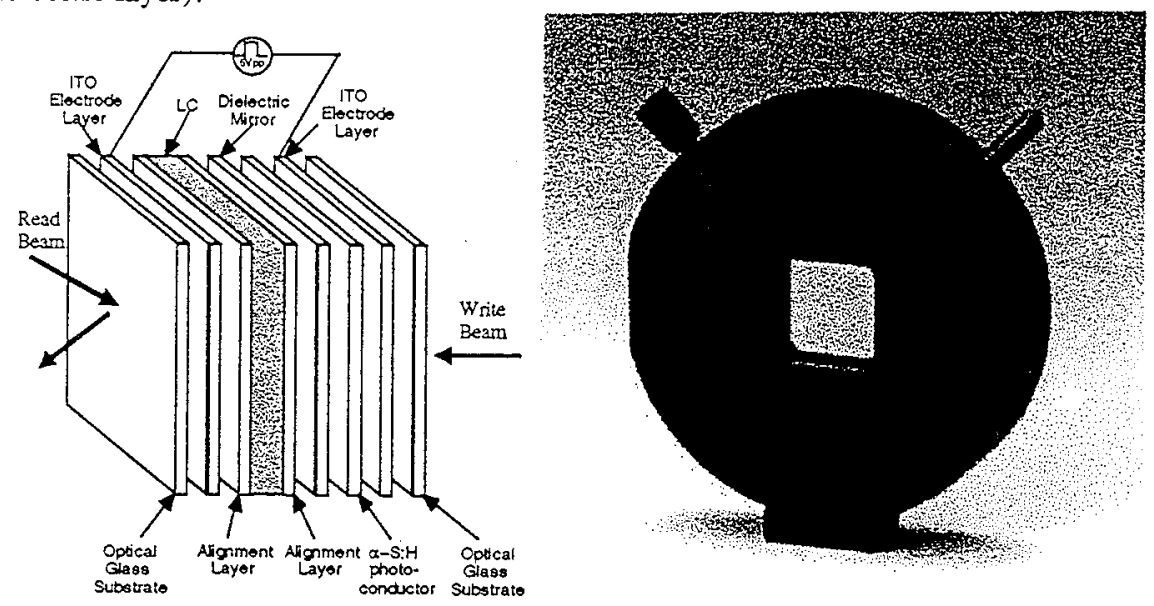

Figure 1. Diagram of the internal structure and photo of the SLM.
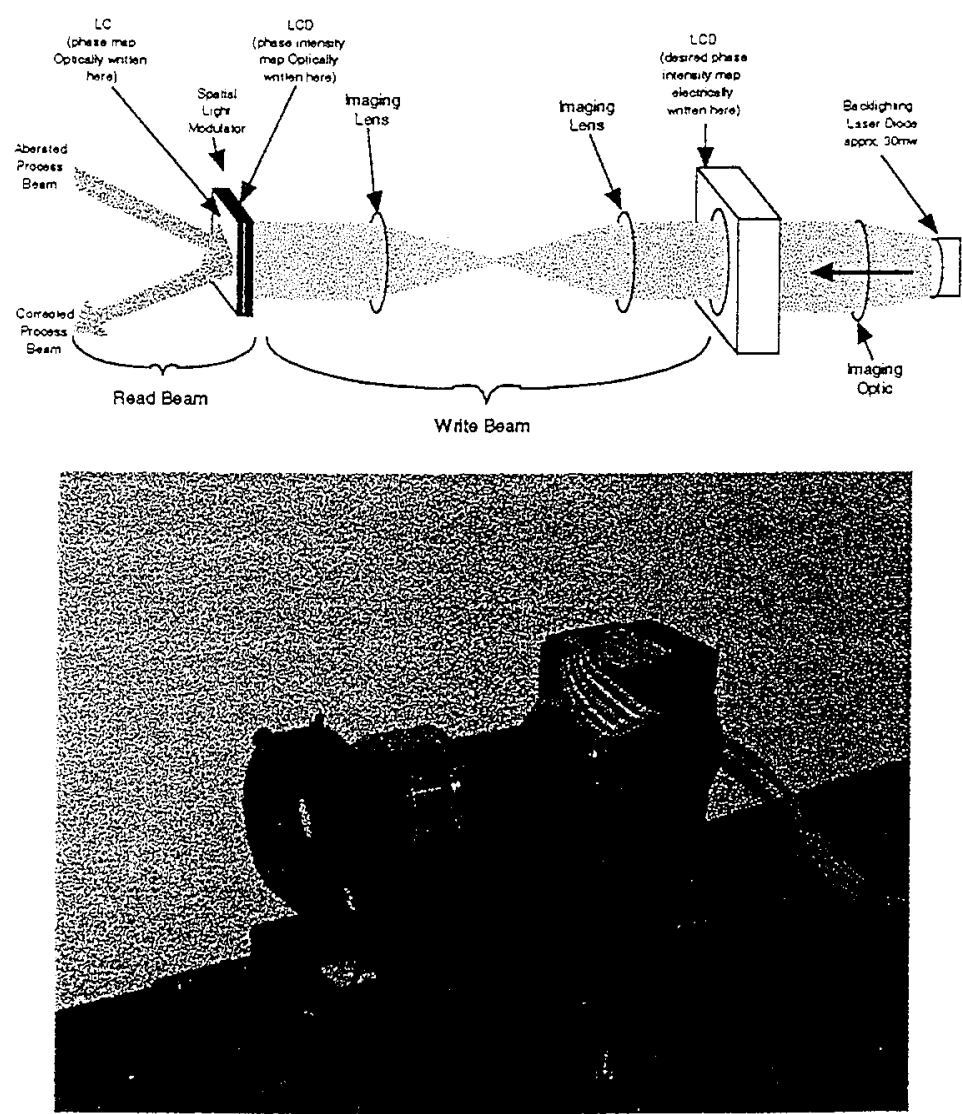

Figure 2. Diagram and photo of the SLM assembly. 
Currently available SLM devices have the capability of providing control of up to 50 line-pairs/mm. The image pattern is written by an LCD and projected onto the SLM. The resolution of the image projection forms the limiting resolution at which the SLM will affect the read beam. The device from Hamamatsu provides a $640 \times 480$ LCD (Sony LCX012BL) having $480 \times 480$ pixels within the $20 \mathrm{~mm} \times 20 \mathrm{~mm}$ active area of the device ${ }^{2}$. The LCD projection optics in the Hamamatsu device incorporate a spatial filter to limit the resolution to approximately $19 \mathrm{Ip} / \mathrm{mm}$. The filter removes the effects of the LCD pixelation (i.e. it minimizes the projected image of the gaps between pixels). The operational configuration is shown in Figure 2. Best performance will be achieved when the back light write beam profile is uniform in intensity. With the limited range of control (approximately 1 wave) a non-uniform back will further degrade the usable range of control.

\section{AO TEST BED}

We have developed an Adaptive Optics test bed to evaluate a variety of high spatial resolution wavefront devices. The test bed, shown in Figure 3, has a variety of instruments to facilitate evaluation. Laser light at $594 \mathrm{~nm}$ is incident upon the device under test, in this case the SLM, at a $22.5^{\circ}$ incidence angle. The reflected light is then split between: 1) a Harmann Sensor, 2) a Near Field Camera, and 3) a far field Sensor. The Hartmann Sensor uses a $200 \mu \mathrm{m}$ pitch, f/25, Adaptive Optics Associates lenslet array. The beam size on the lenslet array yields approximately a $40 \times 50$ sampling of the wavefront, see Figure 4. Both the camera on the Hartmann and far field sensors are $1024 \times 1024,12$ bit, Dalsa CA-D7 CCDs. Current setup allows continuous viewing of the system far field spot concurrent with a far field spot generated by a wavefront reference source. This allows for a constant estimation of the Strehl ratio, and therefore performance, of the system. In addition, there is an optical path for imaging the front face of the SLM (near field sensor). A location exists within the AO testbed conjugate with the front face of the SLM, allowing for the placement of wavefront aberrators, such as a deformable mirror, etc. For visual verification of SLM performance, a Twyman-Green interferometer has been placed into the system (not shown in the figure). This interferometer is bore-sighted to the SLM and views only the phase pattern generated by the SLM. Phase patterns are generated and applied to the LCD in the system via a computer. This pattern is illuminated by a 680nm backlight which is ideally collimated and has a uniform irradiance profile. Our measurements were performed with the backlight running at about $1 \mathrm{~mW} / \mathrm{cm}^{2}$ providing approximately 1 wave of stroke.

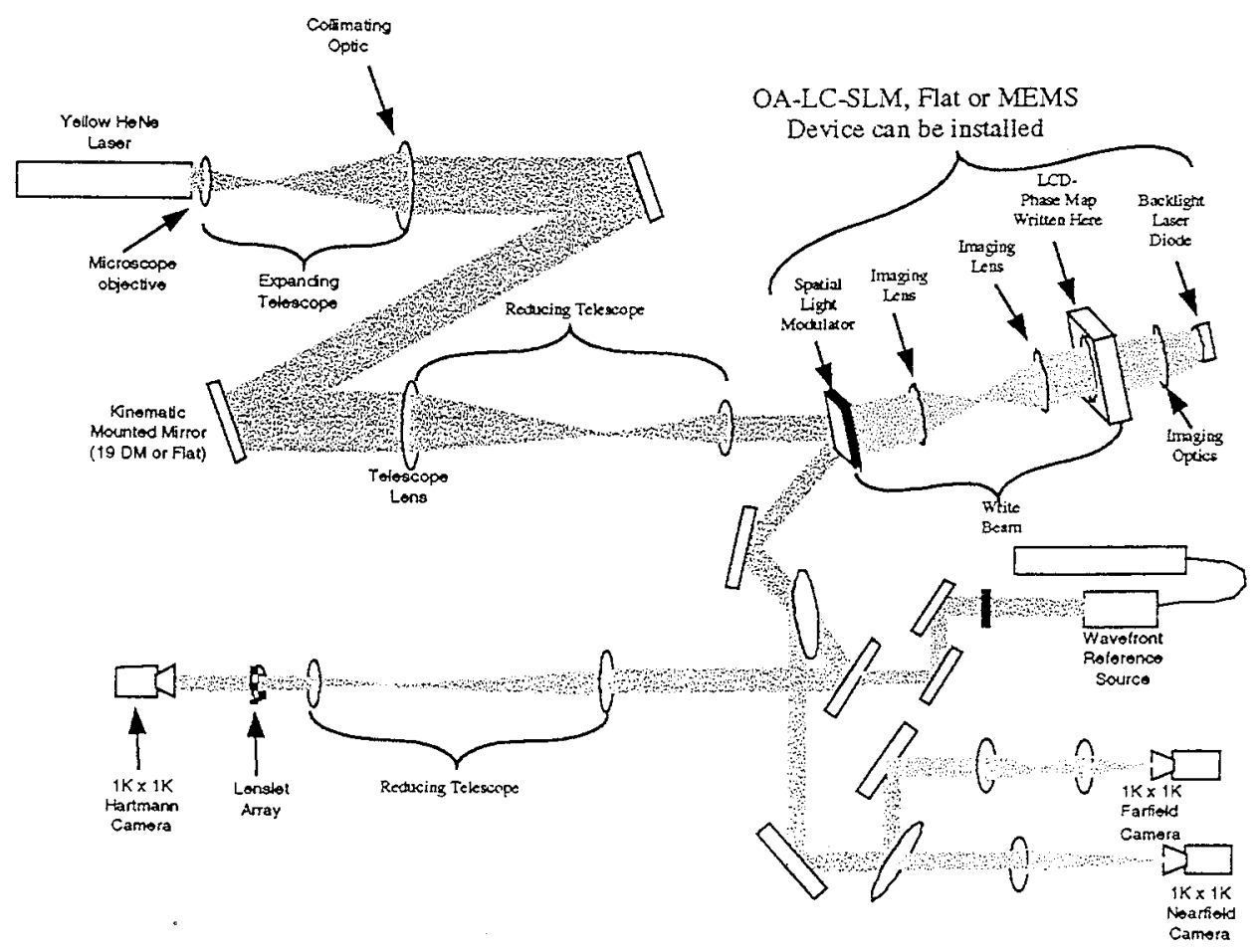

Figure 3. Schematic of the AO Test Bed.

\section{HIGH RESOLUTION WAVEFRONT SENSING AND CONTROL}


The AO Testbed, mentioned above, employs a high resolution Hartmann Sensor (40x50), each aperture 200 microns across with a $5 \mathrm{~mm}$ focal length, see Figure 4 . To reduce computation time, particularly for a closed loop system, new wavefront reconstruction algorithms were developed. These algorithms are based on Fourier modal decomposition and are presented in detail elsewhere ${ }^{3,4}$. The benefit of these algorithms is in the reduction of computational requirements. Instead of the traditional matrix vector multiplication of $O\left(N^{2}\right)$, the FFT approach is $O\left(N \log _{2}(N)\right)$. Speed is appreciably saved as the number of degrees of freedom grows.

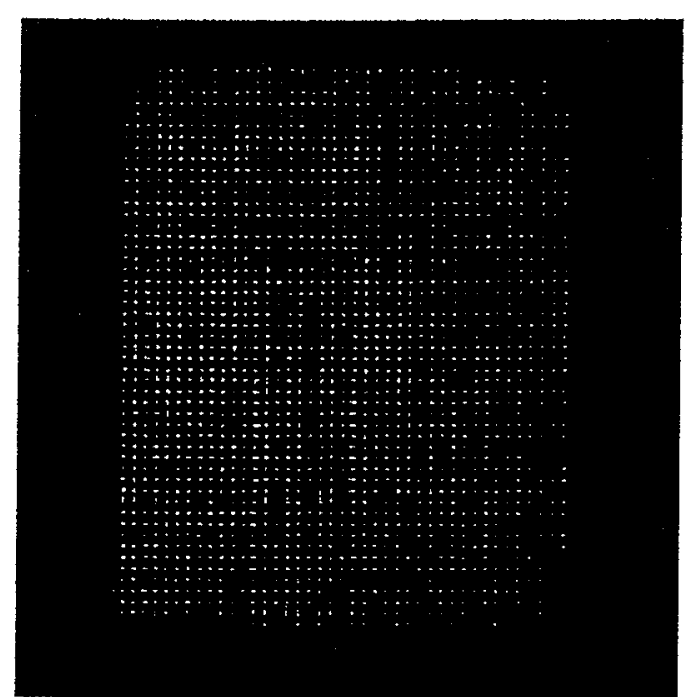

Figure 4. Sample Hartmann Spot reading.

The SLM maintains a significant increase in degrees of freedom over the Hartmann Sensor. Traditional approaches with Deformable mirrors, etc, typically rely on over constraining the system where there are a greater number of sample points than control points. In the current configuration, the Harmann Sensor data is upsampled using interpolation routines. The choice of routines, at this time, is arbitrary and more investigation into effectiveness will be performed. In addition, one can envision decomposing the SLM's $480 \times 480$ control points in to approximately $40 \times 50$ influence functions (Gaussian or otherwise).

The system is currently based on an IDL closed loop controller running at about $1 \mathrm{~Hz}$. IDL was chosen primarily due to its graphics capabilities for displaying information, familiarity for algoritbm development, and the reduction of direct software engineering support required. It has lent itself well to integration with ' $\mathrm{C}$ ' code driving the Hartmann Sensor frame grabber. Ultimately to improve the speed of the closed loop controller, we will run' a primarily ' $\mathrm{C}$ ' based code. Currently, a version of our IDL code has been rewritten in ' $\mathrm{C}$ ' and shows promise at running at the limits of the SLM device, which is between 15 to $25 \mathrm{~Hz}$ due to the required molecular alignment times of the Liquid Crystal molecules. In the present system, however, the Dalsa camera is our limiting feature, running at $8 \mathrm{~Hz}$. Even with binning, the camera is the theoretical limit at $16 \mathrm{~Hz}$.

Figure 5, shows an example measurement performed on the system. A checkerboard pattern 100 pixels wide was applied to the LCD. The corresponding readout as displayed on the interferometer is shown in Figure 5A. The corresponding Hartmann measurement is displayed in Figure 5B. As shown in the figure, the measurements agree qualitatively. Difficulty exists in mapping a LCD intensity to actual wavefront. A sample transfer characteristic curve is shown in Figure 6. 

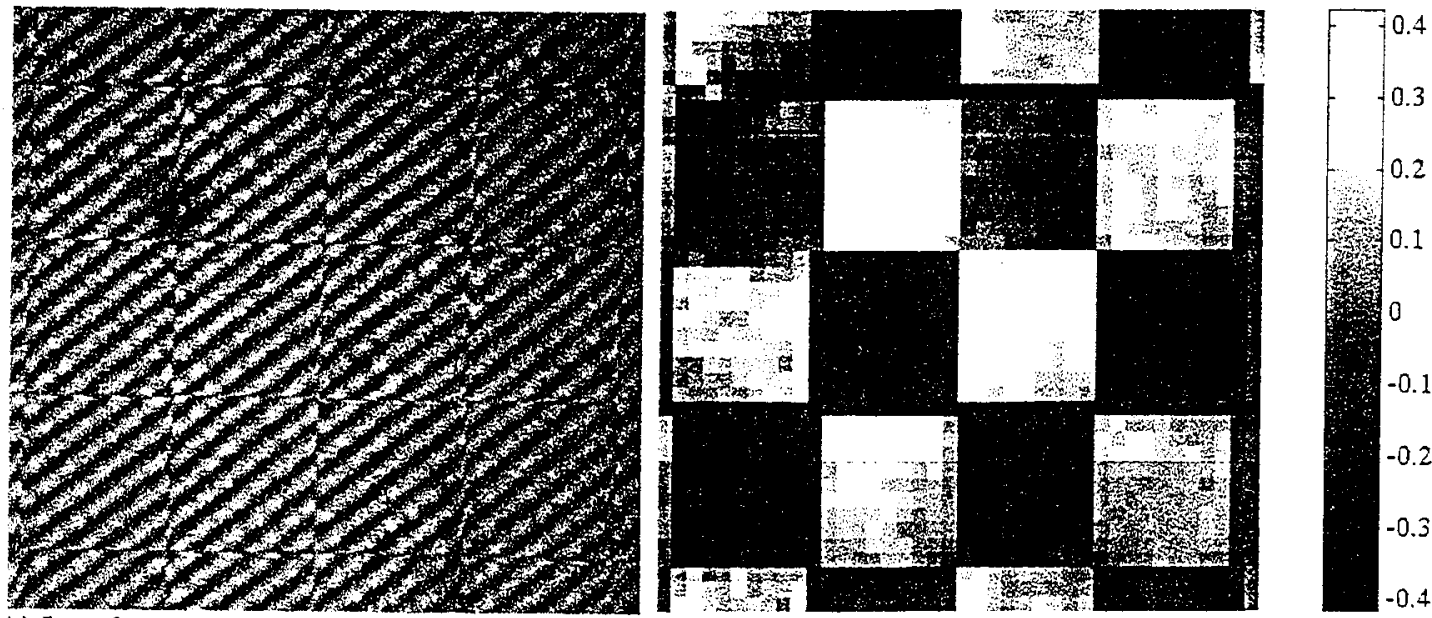

Figure 5, A) Interferometer measurement of a checkerboard pattern applied to the SLM. Note: this picture shows the device driven to approximately $80 \%$ of full wave. B) Corresponding Hartmann Sensor reading.

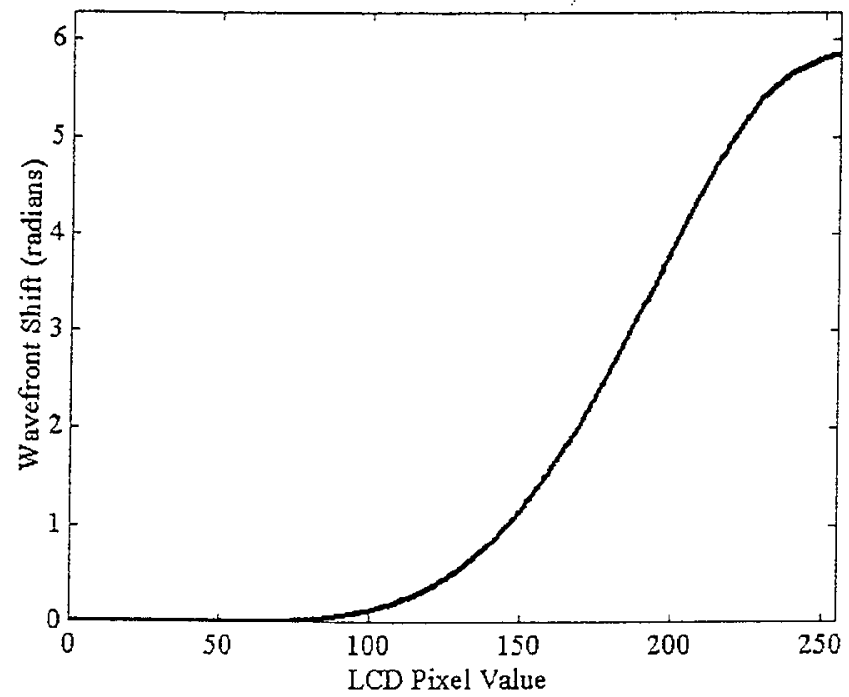

Figure 6. LCD to SLM response curve.

\section{RESULTS OF A CLOSED LOOP ADAPTIVE OPTICS SYSTEM}

To test the ability of the system to converge in a closed loop fashion, we placed a fixed aberration into the system and then allowed the $\mathrm{AO}$ system attempt to minimize wavefront aberration (flatten the wavefront). For this particular test, we placed the aberration onto the SLM itself and had the system attempt to flatten the SLM in a blind fashion (i.e. without knowledge of the pattern applied to it). The aberration placed on the system was a Gaussian modified astigmatic pattern, shown in Figure 7A. The Hartmann sensor reconstruction of this aberration is displayed in Figure 7B. The reconstruction looks reasonably similar to the original aberration. The far field spot generated by the aberration is shown in Figure $7 \mathrm{C}$. The calculated Strehl ratio is 0.27 . The system was run closed loop for 20 iterations with a gain of 0.25 . The resulting wavefront measurement is shown in Figure 7D and the resulting far field is shown in Figure 7E. The resulting measured wavefront was approximately 0.134 radians (or 0.02 waves). The corrected far field spot had a corresponding Strehl of 0.76 . A measurement was performed to determine the flatness of the SLM. The device was removed from the AO testbed and placed in a Wyko interferometer. The measured surface figure is shown in Figure 8. As shown, the RMS aberration is slightly greater the $\lambda / 10$. Closed loop convergence is shown graphically in Figure 9. According to this plot the system shows overdamped convergence. 


\section{CONCLUSIONS}

Currently there are only a handful of options available for high resolution adaptive optics devices. These devices are primarily liquid crystal in nature. There are strong indications that MEMS devices (micro electro-mechanical systems) will eventually play an important role in high resolution $\mathrm{AO}$. As of yet, the devices are just beginning to show promise with high numbers of actuators and good optical quality.

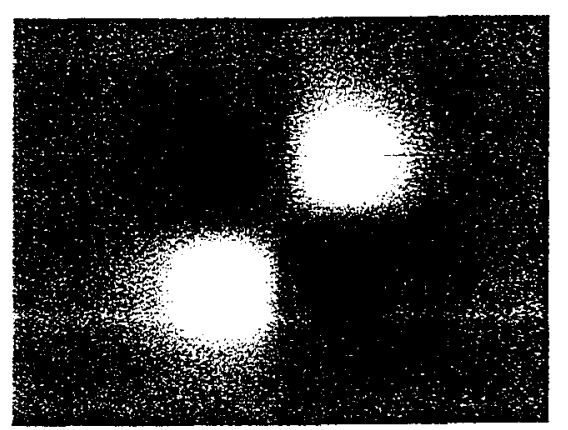

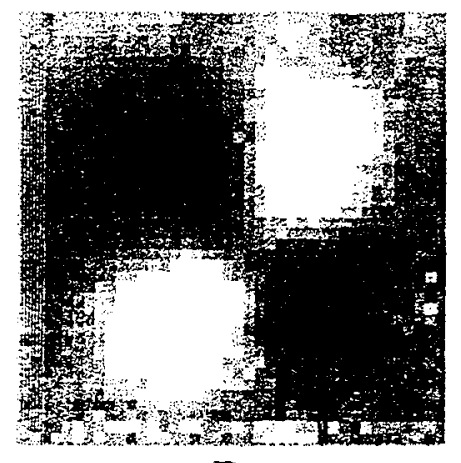

$\mathrm{B}$

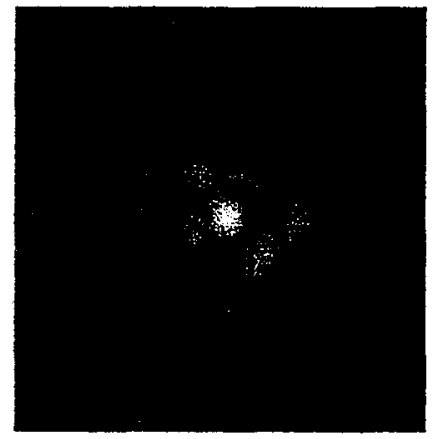

C
A

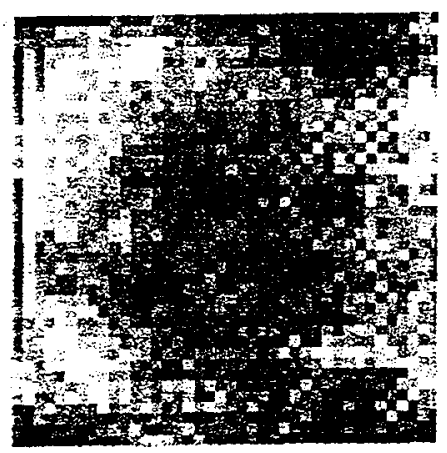

$\mathrm{D}$

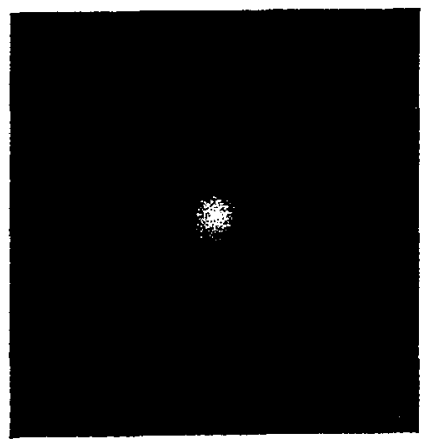

E

Figure 7, Closed Loop performance of AO System. A) Original optical distortion placed on the LCD of the systemB) Original. Hartmann sample wavefront error, C) Corresponding far field spoi (Strehl $=0.27$ ), D) Hartmann sampled wavefront after 20 iterations ( $\mathrm{rms}=0.134$ radians) note: the image was normalized to show wavefront details, E) Corresponding far field spot (Strehl $=0.76)$.

Our current tests show that liquid crystal devices can be used in an application space that has slow temporal phase transitions inherent in them, thus allowing the device to correct for these transitions. Faster temporally aberrated systems will require faster response time liquid crystal of MEMS type devices. Another problem that must be solved by the LC SLM 
manufacturers is in the ability to build devices that have a low residual wavefront. The current LC SLM's have between .25 to .5 waves of residual error, requiring the use of nearly half of the stroke of the device to correct for itself.

Title:

Note:

\section{Contour}

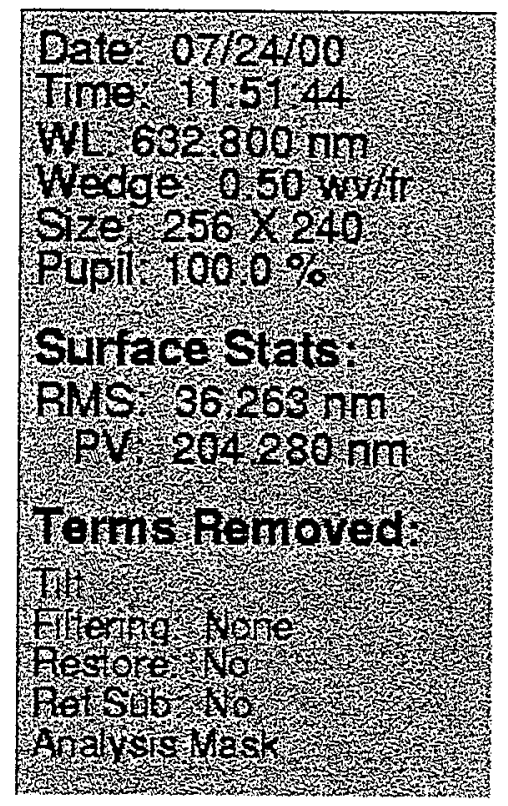

\section{Surface Data}
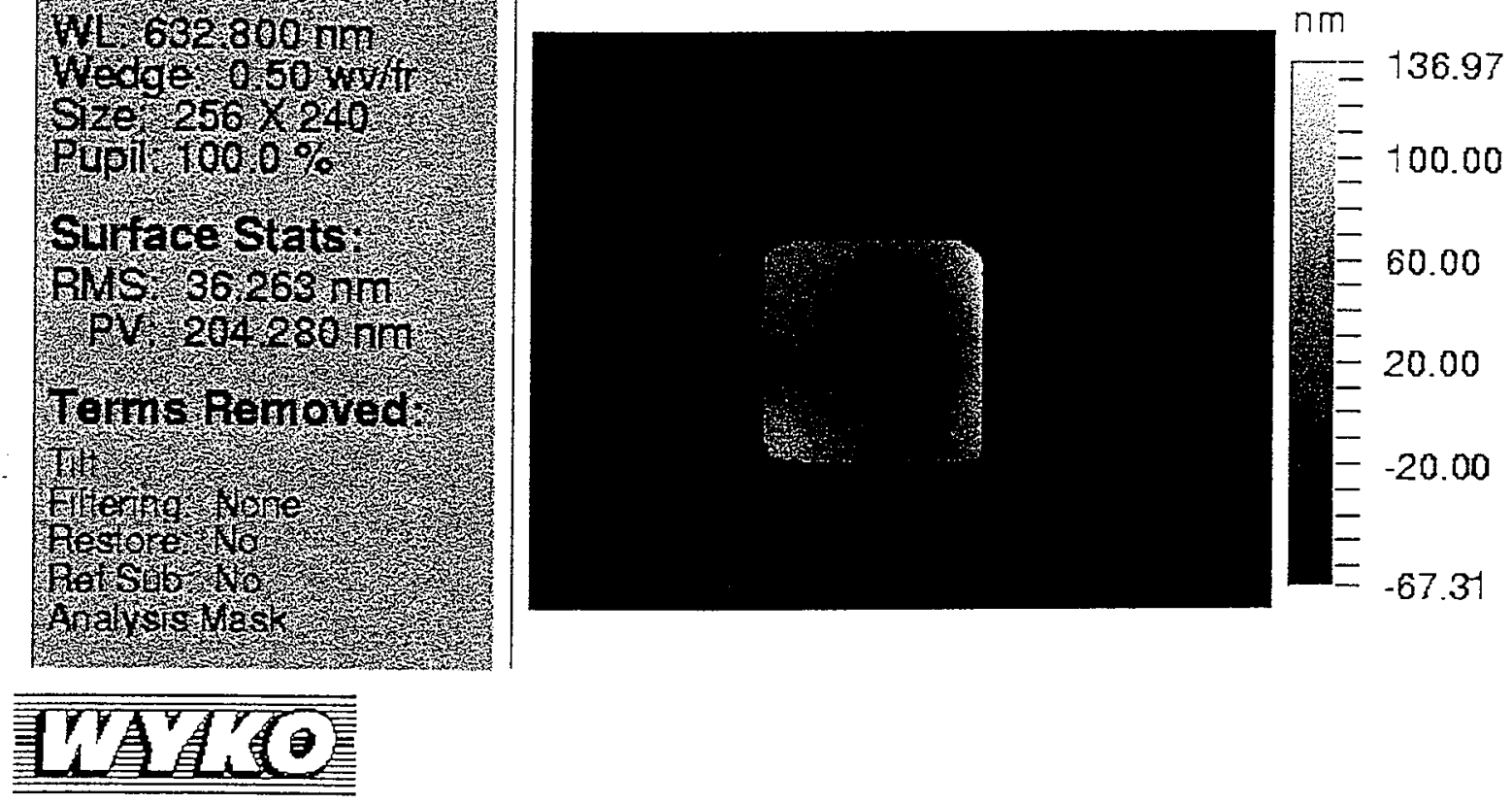

Figure 8, Measurement of the surface figure on the Hamamatsu SLM.

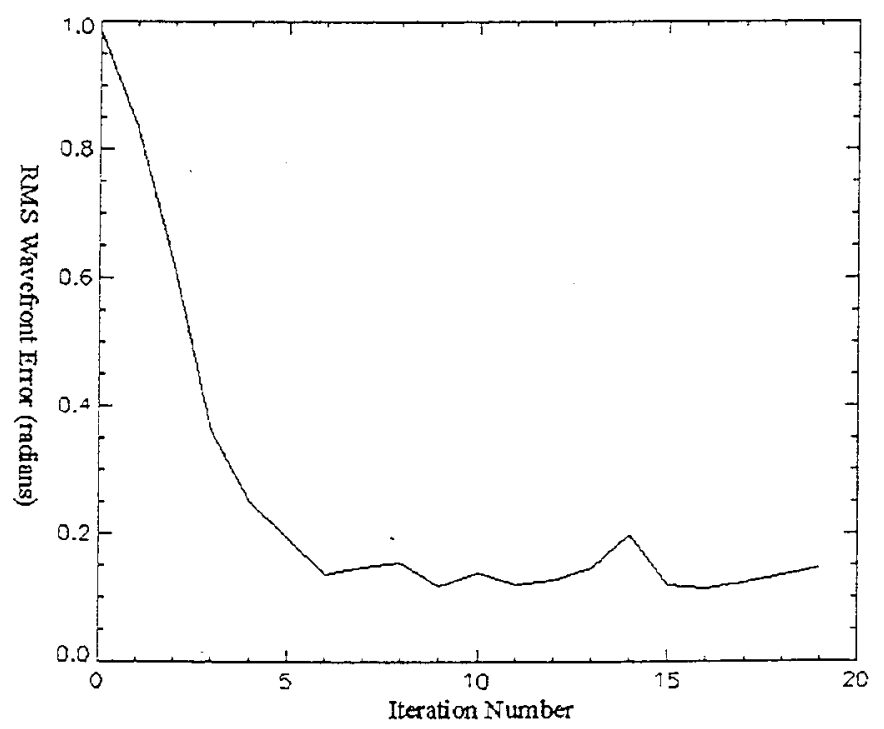

Figure 9, RMS wavefront error versus iteration in the closed loop AO system.

To our knowledge, these are the first quantitative measurements demonstrating near diffraction-limited performance of a wavefront control system using a LC SLM. The combination of the large number of phase-control points and the demonstrated far-field image quality makes this technology a viable choice for high-performance beam control and image 
compensation applications that require high-order correction beyond the capability of conventional deformable mirrors.

\section{REFERENCES}

1. M. W. Kartz, S. S. Olivier, K. Avicola, J. Brase, C. Carrano, D. Silva, D. Pennington, and C. Brown, "High resolution wavefront control of high-power laser systems," Proc. $2^{\text {nd }}$ International Workshop on Adaptive Optics for Industry and Medicine, pp. 16-21, 2000.

2. Y. Kobayashi, Y. Igasaki, N. Yoshida, N. Fukuchi, H. Toyoda, T. Hara, and M. Wu, "Compact high-efficiency electrically-addressable phase-only spatial light modulator", SPIE 3951, 2000.

3. F. U. Dowla, J. Brase, and S. Olivier, "Performance of wavelet and FFT reconstruction algorithms in high-resolution adaptive optics", to be presented, SPIE 4124, 2000.

4. K. Freischlad and C. L. Koliopoulos, "Wavefront reconstruction from noisy slope or difference data using the discrete Fourier transform," SPIE 551, 1985. 


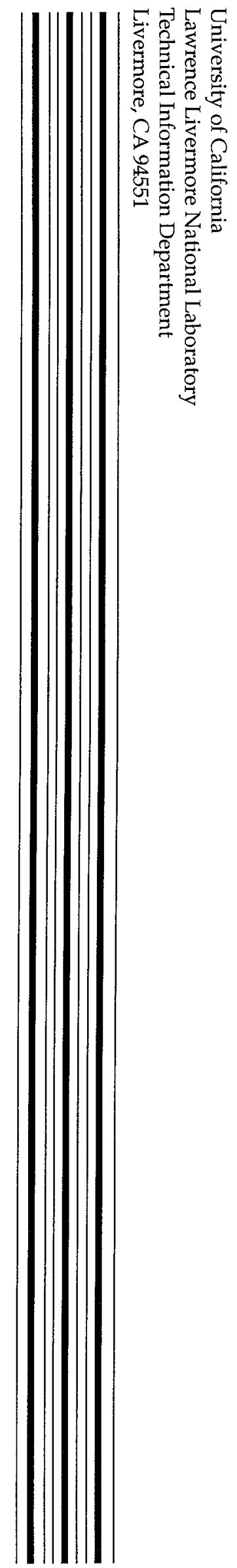

